\title{
The Infinite-message Limit of Two-terminal Interactive Source Coding
}

\author{
Nan Ma and Prakash Ishwar
}

\begin{abstract}
A two-terminal interactive function computation problem with alternating messages is studied within the framework of distributed block source coding theory. For any finite number of messages, a single-letter characterization of the sum-rate-distortion function was established in previous works using standard information-theoretic techniques. This, however, does not provide a satisfactory characterization of the infinite-message limit, which is a new, unexplored dimension for asymptotic-analysis in distributed block source coding involving potentially an infinite number of infinitesimal-rate messages. In this paper, the infinite-message sum-rate-distortion function, viewed as a functional of the joint source pmf and the distortion levels, is characterized as the least element of a partially ordered family of functionals having certain convex-geometric properties. The new characterization does not involve evaluating the infinite-message limit of a finite-message sum-rate-distortion expression. This characterization leads to a family of lower bounds for the infinite-message sum-rate-distortion expression and a simple criterion to test the optimality of any achievable infinite-message sum-rate-distortion expression. For computing the samplewise Boolean AND function of two physically separated independent Bernoulli sources with zero Hamming distortion at one or both terminals, the respective infinite-message minimum sum-rates are characterized in closed analytic form. These sum-rates are shown to be achievable using infinitely many infinitesimal-rate messages. The new convex-geometric characterization is used to develop an iterative algorithm for evaluating any finite-message sumrate-distortion function. It is also used to construct the first examples which demonstrate that for lossy source reproduction, two messages can strictly improve the one-message Wyner-Ziv rate-distortion function settling an unresolved question from a 1985 paper. It is shown that a single backward message of arbitrarily small rate can lead to an arbitrarily large gain in the sum-rate.
\end{abstract}

\section{INTRODUCTION}

In this paper we study a two-terminal interactive function computation problem with alternating messages (Figure 1) within a distributed block source coding framework. Here, $(X(1), Y(1)), \ldots,(X(n), Y(n))$ are $n$ iid samples of a two-component discrete memoryless stationary source with joint pmf $p_{X Y}(x, y),(x, y) \in \mathcal{X} \times \mathcal{Y},|\mathcal{X} \times \mathcal{Y}|<\infty$. The $n$ samples of the first component $\mathbf{X}:=(X(1), \ldots, X(n))$ are available at terminal $A$ whereas the $n$ samples of the second component $\mathbf{Y}:=(Y(1), \ldots, Y(n))$ are available at a different terminal $B$. The two component sources are, in general, statistically dependent. Terminal $A$ is required to produce a sequence $\widehat{\mathbf{Z}}_{A} \in \mathcal{Z}_{A}^{n},\left|\mathcal{Z}_{A}\right|<\infty$, such that

This material is based upon work supported by the US National Science Foundation (NSF) under awards (CAREER) CCF-0546598 and CCF-0915389. Any opinions, findings, and conclusions or recommendations expressed in this material are those of the authors and do not necessarily reflect the views of the NSF. Parts of this work were presented in Alleron'09, ITA'10, and ISIT'10.

N. Ma is with Department of Electrical Engineering and Computer Sciences at the University of California, Berkeley, CA 94709. e-mail: nanma@eecs.berkeley.edu.

P. Ishwar is with the Department of Electrical and Computer Engineering at Boston University, Boston, MA 02215. e-mail: pi@bu.edu. 


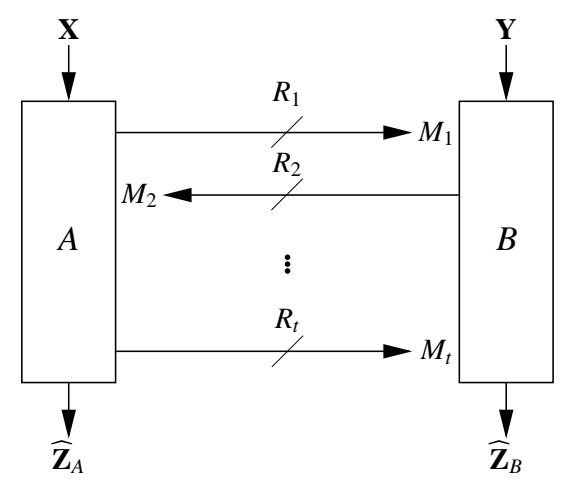

Fig. 1. Interactive distributed block source coding with $t$ alternating messages.

$d_{A}^{(n)}\left(\mathbf{X}, \mathbf{Y}, \widehat{\mathbf{Z}}_{A}\right) \leq D_{A}$ where $d_{A}^{(n)}$ is a distortion function of $3 n$ variables. Similarly, terminal $B$ is required to produce a sequence $\widehat{\mathbf{Z}}_{B} \in \mathcal{Z}_{B}^{n},\left|\mathcal{Z}_{B}\right|<\infty$, such that $d_{B}^{(n)}\left(\mathbf{X}, \mathbf{Y}, \widehat{\mathbf{Z}}_{B}\right) \leq D_{B}$. To achieve the desired objective, $t$ coded messages, $M_{1}, \ldots, M_{t}$, of respective bit rates (bits per source sample), $R_{1}, \ldots, R_{t}$, are sent alternately from the two terminals starting with some terminal. Each message sent from a terminal can depend on the source samples at that terminal and on all the previous messages (which are available to both terminals). There is enough memory at both terminals to store all the source samples and messages. After $t$ messages, terminal $A$ produces a sequence $\widehat{\mathbf{Z}}_{A} \in \mathcal{Z}_{A}^{n}$ and terminal $B$ produces a sequence $\widehat{\mathbf{Z}}_{B} \in \mathcal{Z}_{B}^{n}$. The sum-rate-distortion function $R_{\text {sum }, t}\left(D_{A}, D_{B}\right)$ is the infimum of the sum of all rates $\sum_{i=1}^{t} R_{i}$ for which the following criteria hold: $\mathbb{P}\left(d_{A}^{(n)}\left(\mathbf{X}, \mathbf{Y}, \widehat{\mathbf{Z}}_{A}\right)>D_{A}\right)$ and $\mathbb{P}\left(d_{B}^{(n)}\left(\mathbf{X}, \mathbf{Y}, \widehat{\mathbf{Z}}_{B}\right)>D_{B}\right)$ $\rightarrow 0$ as $n \rightarrow \infty$.

When the distortion criterion is of the form of vanishing probability of block-error (Section 【), or of the form of expected per-sample distortion (Section VII-A), for any finite number $t$, a single-letter characterization of the set of all feasible coding rate-distortion tuples (the rate-distortion region) and the $t$-message sum-rate-distortion function $R_{\text {sum }, t}\left(D_{A}, D_{B}\right)$, was established in previous works [1], [2], in terms of minimizing certain conditional mutual information quantities involving auxiliary random variables satisfying certain conditional independence and cardinality constraints. This does not, in general, provide a satisfactory characterization of the infinite-message limit $R_{\text {sum }, \infty}\left(D_{A}, D_{B}\right):=\lim _{t \rightarrow \infty} R_{\text {sum }, t}\left(D_{A}, D_{B}\right)$. The two main objectives of this paper are to provide: (i) a characterization of $R_{\text {sum }, \infty}\left(D_{A}, D_{B}\right)$ which is not in terms of evaluating the infinite-message limit of a finite-message sum-ratedistortion expression, and (ii) an iterative algorithm to evaluate it. Understanding the sum-rate-distortion function in the limit where potentially an infinite number of alternating messages are allowed to be exchanged will shed light on the fundamental benefit of cooperative interaction in two-terminal problems. While asymptotics involving blocklength, rate, quantizer step-size, and network size have been explored in the distributed block source coding literature, asymptotics involving an infinite number of messages, each with potentially infinitesimal rate, has not been studied. The number of messages is a relatively unexplored resource and a new dimension for asymptotic analysis.

\section{A. Contributions}

By viewing the sum-rate-distortion function as a functional of the joint source distribution and distortion levels, a new convex-geometric blocklength-free single-letter characterization of the infinite-message sum-rate-distortion function is developed. The new characterization is "limit-free" in that it does not involve evaluating an infinite- 
message limit. Instead, it is in terms of the least element of a family of partially-ordered functionals defined by the coupled per-sample distortion criteria. The new characterization is free of auxiliary random variables and therefore does not involve any Markov chain constraints or cardinality bounds. The new characterization leads to a simple criterion to test the optimality of any achievable infinite-message sum-rate-distortion expression.

For computing, with zero Hamming distortion, the samplewise Boolean AND function of two independent Bernoulli component sources at one or both terminals, the respective infinite-message minimum sum-rates are fully characterized in closed analytic form. These optimal sum-rates are shown to be achievable using a novel construction for an infinite sequence of auxiliary random variables that, in the limit, correspond to using infinitely many infinitesimal-rate messages.

The functional viewpoint is used to develop an iterative algorithm for evaluating any finite-message sum-ratedistortion function which includes, as special cases, the rate-distortion, conditional rate-distortion, and Wyner-Ziv rate-distortion functions. In the algorithm, the complexity of computation in each iteration does not grow with iteration number.

The new convex-geometric characterization is also used to affirmatively answer the following question that was left unresolved in [3]: For lossy source reproduction, can the two-message sum-rate-distortion function be strictly smaller than the one-message Wyner-Ziv rate-distortion function? Explicit examples are constructed to demonstrate that the ratio and the difference between the one-message and two-message rate-distortion functions can be arbitrarily large and simultaneously the ratio of the backward rate to the forward rate in the two-message sum-rate can be arbitrarily small. These are the first known examples that explicitly demonstrate the benefit of interaction for distributed lossy source reproduction.

Results for the sum-rate-distortion function are also extended to the weighted sum-rate-distortion function where the rates of messages sent from $A$ to $B$ and from $B$ to $A$ are weighted to account for communication costs that are different in different directions. The weighted sum-rate-distortion function can be further used to characterize the directed sum-rate-distortion region, which represents the tradeoff between the two directed sum-rates from $A$ to $B$ and from $B$ to $A$, and the target distortion levels.

\section{B. Related work}

Related interactive computation problems have been studied extensively in the area of communication complexity [4], [5] where the main focus is on exact (error-free) computation, without regard for the statistical dependencies in samples across terminals, and where computing efficiency is gauged in terms of the order-of-magnitude of the total number of bits exchanged; not bit-rate (notable exceptions to this main focus are [6], [7]). Two-way distributed block source coding where the goal is to reproduce the sources with a non-zero per-sample distortion, as opposed to computing functions, was studied by Kaspi [3] who characterized the $t$-message sum-rate-distortion function in each direction. Orlitsky and Roche [8] studied two-terminal samplewise function computation with a vanishing block-error probability and characterized the feasible rates and the minimum sum-rate for two alternating messages $(t=2)$. A more detailed account of related work appears in [2].

In [9], the infinite message limit of the minimum sum-rate for function computation was studied for a collocated network. The problem formulation there differs from that in this paper in the following ways. (i) A collocated network contains multiple source nodes and a sink node that has no observations, whereas the two-terminal problem network there is no sink node. (ii) In a collocated network the topology of communication is noiseless broadcast, 
(iii) The sources are mutually independent in [9], but are allowed to be arbitrarily related here. (iv) The function computation is lossless in [9] but is allowed to be lossy here. Due to these differences, the results in this paper are not corollaries of the results in [9] and vice versa. The methodology in Sections III] and IV] is similar to the counterparts in [9] in spirit but is for a totally different problem.

\section{Paper-organization and notation}

For clarity of exposition, results are first developed for the lossless function computation problem with the vanishing probability of block-error criterion 1 in Sections [I-VI In Section II, after formulating the problem, the key results from [1], [2] that are needed for the subsequent development are summarized. The new convexgeometric characterization of the infinite-message minimum sum-rate is developed in Section III An iterative algorithm for evaluating any finite-message minimum sum-rate is then developed in Section IV The infinite-message minimum sum-rates for two examples are evaluated in closed analytic form in Section $\mathrm{V}$ The characterizations of the weighted minimum sum-rate and the directed sum-rate region are presented in Section VI In Section VII, the results for lossless computation are generalized to the lossy rate-distortion problem with the per-sample distortion criterion. Then, in Section VIII we use the tools developed in Section VII to demonstrate that in distributed lossy source reproduction problems, two-message interaction can significantly improve the non-interactive Wyner-Ziv rate-distortion function.

Vectors are denoted by boldface letters; the dimension will be clear from the context. The acronym 'iid' stands for independent and identically distributed and 'pmf' stands for probability mass function. With the exception of the symbols $R, D, N, A$, and $B$, random quantities are denoted in upper case and their specific instantiations in lower case. For integers $i, j$, with $i \leq j, V_{i}^{j}$ denotes the sequence of random variables $V_{i}, \ldots, V_{j}$. For $i \geq 1, V_{1}^{i}$ is abbreviated to $V^{i}$. If $j<i$ then " $V_{i}^{j}$ " is the void expression "". More generally, if a quantity $Q_{i}$ is defined only for indices $i$ that belong to a subset $\mathcal{S}$ of integers then for all integers $i$ not in $\mathcal{S}$, " $Q_{i}$ " = "”. For a set $\mathcal{S}, \mathcal{S}^{n}$ denotes the $n$-fold Cartesian product $\mathcal{S} \times \ldots \times \mathcal{S}$. The support-set of a pmf $p$ is the set over which it is strictly positive and is denoted by $\operatorname{supp}(p)$. If $\operatorname{supp}(q) \subseteq \operatorname{supp}(p)$ then we write $q \ll p$. The set of all pmfs on alphabet $\mathcal{A}$, i.e., the probability simplex in $\mathbb{R}^{\mid \mathcal{A l}}$, is denoted by $\Delta(\mathcal{A})$. $X \sim \operatorname{Ber}(p)$ means $p_{X}(1)=1-p_{X}(0)=p$, and $h_{2}(p)$ denotes its entropy in bits. Unif $_{\mathcal{A}}$ denotes the uniform distribution in the set $\mathcal{A}$. $X \Perp Y$ means $X$ and $Y$ are independent. The indicator function of set $\mathcal{S}$ which is equal to one if $x \in \mathcal{S}$ and is zero otherwise, is denoted by $1_{\mathcal{S}}(x)$. Symbols $\wedge$ and superscript $c$ represent Boolean AND and complement respectively. A function $f: \mathcal{A} \rightarrow \mathbb{R}$ is said to majorize another function $g: \mathcal{A} \rightarrow \mathbb{R}, f$ if $\forall x \in \mathcal{A}, f(x) \geq g(x)$. The hypograph of a function $f(x)$ on a set $\mathcal{A}$ is given by $\operatorname{hypo}_{\mathcal{A}} f:=\{(x, \phi): x \in \mathcal{A}, \phi \leq f(x)\}$. The convex hull of a set $\mathcal{A}$ is denoted by $\operatorname{ch}(\mathcal{A})$. For any $a \in[0,1], \bar{a}:=1-a$. For the erasure symbol $e, \bar{e}:=e$.

\section{INTERACTIVE FUNCTION COMPUTATION PROBLEM}

Let $f_{A}: \mathcal{X} \times \mathcal{Y} \rightarrow \mathcal{Z}_{A}$ and $f_{B}: \mathcal{X} \times \mathcal{Y} \rightarrow \mathcal{Z}_{B}$ be functions of interest at terminals $A$ and $B$ respectively. The desired outputs at terminals $A$ and $B$ are $\mathbf{Z}_{A}$ and $\mathbf{Z}_{B}$ respectively, where for $i=1, \ldots, n, Z_{A}(i):=f_{A}(X(i), Y(i))$ and $Z_{B}(i):=f_{B}(X(i), Y(i))$

\footnotetext{
${ }^{1}$ If the per-sample distortion function is chosen to be the Hamming distortion with respect to a function of $X$ and $Y$ and the distortion level is set to zero, the characterization of the sum-rate-distortion function essentially reduces to the characterization of the minimum sum-rate for computing a function of $X$ and $Y$ with a vanishing probability of block-error [2. Proposition 3]. In this sense, the per-sample distortion criterion is more general than the vanishing probability of block error criterion.
} 
A two-terminal interactive distributed source code (for function computation) with initial terminal $A$ and parameters $\left(t, n,\left|\mathcal{M}_{1}\right|, \ldots,\left|\mathcal{M}_{t}\right|\right)$ is the tuple $\left(e_{1}, \ldots, e_{t}, g_{A}, g_{B}\right)$ of $t$ block encoding functions $e_{1}, \ldots, e_{t}$ and two block decoding functions $g_{A}, g_{B}$, of blocklength $n$, where for $j=1, \ldots, t$,

$$
\begin{aligned}
& \text { (Enc.j) } e_{j}: \begin{cases}X^{n} \times \bigotimes_{i=1}^{j-1} \mathcal{M}_{i} \rightarrow \mathcal{M}_{j}, & \text { if } j \text { is odd } \\
\mathcal{Y}^{n} \times \bigotimes_{i=1}^{j-1} \mathcal{M}_{i} \rightarrow \mathcal{M}_{j}, & \text { if } j \text { is even }\end{cases} \\
& \text { (Dec.A) } g_{A}: X^{n} \times \bigotimes_{j=1}^{t} \mathcal{M}_{j} \rightarrow Z_{A}^{n}, \\
& \text { (Dec.B) } g_{B}: \quad \boldsymbol{y}^{n} \times \bigotimes_{j=1}^{t} \mathcal{M}_{j} \rightarrow Z_{B}^{n} .
\end{aligned}
$$

The output of $e_{j}$, denoted by $M_{j}$, is called the $j$-th message, and $t$ is the number of messages. The outputs of $g_{A}$ and $g_{B}$ are denoted by $\widehat{\mathbf{Z}}_{A}$ and $\widehat{\mathbf{Z}}_{B}$ respectively. For each $j,(1 / n) \log _{2}\left|\mathcal{M}_{j}\right|$ is called the $j$-th block-coding rate (in bits per sample). The sum of all the individual rates $(1 / n) \sum_{j=1}^{t} \log _{2}\left|\mathcal{M}_{j}\right|$ is called the sum-rate.

A rate tuple $\mathbf{R}=\left(R_{1}, \ldots, R_{t}\right)$ is admissible for $t$-message interactive function computation with initial terminal $A$ if, $\forall \epsilon>0, \exists N(\epsilon, t)$ such that $\forall n>N(\epsilon, t)$, there exists an interactive distributed source code with initial terminal $A$ and parameters $\left(t, n,\left|\mathcal{M}_{1}\right|, \ldots,\left|\mathcal{M}_{t}\right|\right)$ satisfying

$$
\begin{aligned}
& \frac{1}{n} \log _{2}\left|\mathcal{M}_{j}\right| \leq R_{j}+\epsilon, j=1, \ldots, t, \\
& \mathbb{P}\left(\mathbf{Z}_{A} \neq \widehat{\mathbf{Z}}_{A}\right) \leq \epsilon, \mathbb{P}\left(\mathbf{Z}_{B} \neq \widehat{\mathbf{Z}}_{B}\right) \leq \epsilon
\end{aligned}
$$

We note that of interest here are the probabilities of block error $\mathbb{P}\left(\mathbf{Z}_{A} \neq \widehat{\mathbf{Z}}_{A}\right)$ and $\mathbb{P}\left(\mathbf{Z}_{B} \neq \widehat{\mathbf{Z}}_{B}\right)$ which are multiletter distortion functions. The set of all admissible rate tuples, denoted by $\mathcal{R}_{t}^{A}$, is called the operational rate region for $t$-message interactive function computation with initial terminal $A$. The rate region is closed and convex due to the way it has been defined. The minimum sum-rate is given by

$$
R_{\text {sum }, t}^{A}=\min _{\mathbf{R} \in \mathcal{R}_{t}^{A}} \sum_{j=1}^{t} R_{j} .
$$

For initial terminal $B$, the rate region and the minimum sum-rate are denoted by $\mathcal{R}_{t}^{B}$ and $R_{\text {sum }, t}^{B}$ respectively.

We allow the number of messages $t$ to be equal to 0 . When $t=0$, there is no message transfer and the initial terminal is irrelevant. Thus for $t=0$, in the notation for the minimum sum-rate, we omit the superscript and denote the minimum sum-rate by $R_{\text {sum }, 0}$.

For a given initial terminal, for $t=0$ and $t=1$, function computation may not be feasible for general $p_{X Y}, f_{A}$, $f_{B}$. If the computation is infeasible, $\mathcal{R}_{t}^{A}$ is empty and we set $R_{\text {sum }, t}^{A}=+\infty$. If for some specific $p_{X Y}, f_{A}, f_{B}$, the computation is feasible, then $R_{\text {sum }, t}^{A}$ will be finite. We note that for $t \geq 2$, the computation is always feasible and $R_{\text {sum }, t}^{A}$ is finite.

For all $j \leq t$, null messages, i.e., messages for which $\left|\mathcal{M}_{j}\right|=1$, are permitted. Hence, a $(t-1)$-message interactive code is a special case of a $t$-message interactive code. Thus, $R_{\text {sum },(t-1)}^{A} \geq R_{\text {sum }, t}^{A}$ and $R_{\text {sum },(t-1)}^{A} \geq R_{\text {sum }, t}^{B}$ (see [1], Proposition 1] for a detailed discussion). Therefore, $\lim _{t \rightarrow \infty} R_{\text {sum }, t}^{A}=\lim _{t \rightarrow \infty} R_{\text {sum }, t}^{B}=: R_{\text {sum }, \infty}$. The limit $R_{\text {sum }, \infty}$ is the infinite-message minimum sum-rate.

Depending on the specific joint source pmf $p_{X Y}$ and functions $f_{A}$ and $f_{B}$, it may be possible to reach the infinitemessage limit $R_{\text {sum }, \infty}$ with finite $t$ (see end of Section $\bigvee-\mathrm{B}$ for an example). 
For all finite $t$, a single-letter characterization of the operational rate region $\mathcal{R}_{t}^{A}$ and the minimum sum-rate $R_{\text {sum }, t}^{A}$ were respectively provided in Theorem 1 and Corollary 1 of [1].

Fact 1: (Characterization of $R_{\text {sum }, t}^{A}$ [1] Corollary 1])

$$
R_{\text {sum }, t}^{A}=\min _{p_{U^{t} \mid X Y} \in \mathcal{P}_{t}^{A}\left(p_{X Y}\right)}\left[I\left(X ; U^{t} \mid Y\right)+I\left(Y ; U^{t} \mid X\right)\right] .
$$

where $\mathcal{P}_{t}^{A}\left(p_{X Y}\right)$ is the set of all $p_{U^{t} \mid X Y}$ such that (i) $H\left(f_{A}(X, Y) \mid X, U^{t}\right)=H\left(f_{B}(X, Y) \mid Y, U^{t}\right)=0$, (ii) for $i=1, \ldots, t$, if $i$ is odd, $U_{i}-\left(X, U^{i-1}\right)-Y$ forms a Markov chain, otherwise $U_{i}-\left(Y, U^{i-1}\right)-X$ forms a Markov chain, and (iii) $\mathcal{U}_{1}, \ldots, \mathcal{U}_{t}$ are finite alphabets whose cardinalities are bounded as follows

$$
\left|\mathcal{U}_{j}\right| \leq \begin{cases}|\mathcal{X}|\left(\prod_{i=1}^{j-1}\left|\mathcal{U}_{i}\right|\right)+t-j+3, & j \text { odd } \\ |\mathcal{Y}|\left(\prod_{i=1}^{j-1}\left|\mathcal{U}_{i}\right|\right)+t-j+3, & j \text { even }\end{cases}
$$

Since the constraint set of (1) is a compact set in a finite dimensional Euclidean space and the objective function is continuous, a minimizer exists in $\mathcal{P}_{t}^{A}\left(p_{X Y}\right)$ and (1) is a finite dimensional optimization problem. Although the characterization of the minimum sum-rate in (1) does not explicitly provide an actual code, it can be achieved by a sequence of "Wyner-Ziv like" codes that are based on random coding and binning. The optimality of this coding strategy, i.e., the converse, is proved using standard information inequalities, in particular Fano's inequality, suitably defining auxiliary random variables in terms of source components and message variables, and using Carathéodory's theorem to establish bounds on the cardinalities of the auxiliary random variables.

The conditional entropy constraints $H\left(f_{A}(X, Y) \mid X, U^{t}\right)=H\left(f_{B}(X, Y) \mid Y, U^{t}\right)=0$ allow us to almost completely abstract out the function computation aspects of the problem and focus on the rates. This bears resemblance to the formulation of rate regions in the network coding literature (cf. [10] and [11, Chapter 21]).

The characterization of $R_{\text {sum }, t}^{A}$ in (1) does not directly inform us how quickly $R_{\text {sum }, t}^{A}$ converges to $R_{\text {sum, }, \infty}$, i.e., bounds on the rate of convergence are unavailable for general $p_{X Y}, f_{A}$, and $f_{B}$. In the absence of such bounds, one pragmatic approach to estimate $R_{\text {sum }, \infty}$ is to compute $R_{\text {sum }, t}^{A}$ for increasing values of $t$ - by using a computer to numerically solve (with some computer precision) the finite-dimensional optimization problem in (1) - until the difference between $R_{\text {sum, } t-1}^{A}$ and $R_{\text {sum }, t}^{A}$ is smaller than some small number. Although (1) provides a single-letter characterization for $R_{\text {sum }, t}^{A}$ for each finite $t$, as $t$ increases, an increasing number of auxiliary random variables $U^{t}$ are involved in the optimization problem. In fact, due to (2), the upper bounds for $\left|\mathcal{U}_{t}\right|$ increase exponentially with respect to $t$. Therefore, the dimension of the optimization problem in (1) explodes as $t$ increases. Each iteration is computationally much more demanding than the previous one. To make matters worse, there appears to be no obvious way of re-using the computations done for evaluating $R_{\text {sum }, t-1}^{A}$ when evaluating $R_{\text {sum, },}^{A}$, i.e., every time $t$ is increased, a new optimization problem needs to be solved all over again. Finally, if we need to estimate $R_{\text {sum, }}$ for a different joint source pmf $p_{X Y}$ (but for the same functions $f_{A}$ and $f_{B}$ ), we would need to repeat this entire process for the new $p_{X Y}$.

\section{Characterization of $\boldsymbol{R}_{\text {sum }, \infty}\left(p_{X Y}\right)$}

In this section, we take a new fundamentally different approach. We develop a general blocklength-free characterization of $R_{\text {sum }, \infty}$ which does not involve taking a limit as $t \rightarrow \infty$. Instead of developing the characterization of $R_{\text {sum }, \infty}$ for a fixed joint source pmf $p_{X Y}$ - which is a single nonnegative real number - we characterize the entire infinite-message minimum sum-rate surface $R_{s u m, \infty}\left(p_{X Y}\right)$ - which is a functional of the joint source pmf $p_{X Y}-$ in 
a single concise description. This leads to a simple test for checking if a given achievable sum-rate functional of $p_{X Y}$ coincides with $R_{\text {sum }, \infty}\left(p_{X Y}\right)$. It also provides a whole new family of lower bounds for $R_{\text {sum }, \infty}$.

The key new object needed to develop the new characterization is the rate-reduction functional.

\section{A. The rate reduction functional $\rho_{t}^{A}\left(p_{X Y}\right)$}

If the goal is to losslessly reproduce the sources, i.e., $f_{A}(x, y)=y, f_{B}(x, y)=x$, the minimum sum-rate is equal to $H(X \mid Y)+H(Y \mid X)$ and this can be achieved by Slepian-Wolf coding. The sum-rate needed for computing functions can only be smaller than that needed for reproducing sources losslessly. The reduction in the minimum sum-rate for function computation in comparison to source reproduction is given by

$$
\rho_{t}^{A}:=H(X \mid Y)+H(Y \mid X)-R_{\text {sum }, t}^{A}=\max _{p_{U^{t} \mid X Y} \in \mathcal{P}_{t}^{A}\left(p_{X Y}\right)}\left[H\left(X \mid Y, U^{t}\right)+H\left(Y \mid X, U^{t}\right)\right] .
$$

For interactive distributed source codes with initial terminal $B$, the minimum sum-rate and rate reduction are denoted by $R_{\text {sum }, t}^{B}$ and $\rho_{t}^{B}$ respectively. A quantity which plays a key role in the characterization of $R_{\text {sum }, \infty}$ is $\rho_{0}^{A}$ corresponding to the "rate reduction" for zero messages (there are no auxiliary random variables in this case). Since the initial terminal has no significance when $t=0, \rho_{0}^{A}=\rho_{0}^{B}=: \rho_{0}$. Let

$$
\mathcal{P}_{f_{A} f_{B}}:=\left\{p_{X Y} \in \Delta(X \times \mathcal{Y}): H\left(f_{A}(X, Y) \mid X\right)=H\left(f_{B}(X, Y) \mid Y\right)=0\right\}
$$

Error-free computations can be performed without any message transfers if, and only if, $p_{X Y} \in \mathcal{P}_{f_{A} f_{B}}$. Thus,

$$
\begin{gathered}
R_{\text {sum }, 0}=\left\{\begin{array}{cc}
0, & \text { if } p_{X Y} \in \mathcal{P}_{f_{A} f_{B}}, \\
+\infty, & \text { otherwise, }
\end{array}\right. \\
\rho_{0}=\left\{\begin{array}{cc}
H(X \mid Y)+H(Y \mid X), & \text { if } p_{X Y} \in \mathcal{P}_{f_{A} f_{B}}, \\
-\infty, & \text { otherwise. }
\end{array}\right.
\end{gathered}
$$

Remark 1: If $f_{A}(x, y)$ is not a function of $x$ alone and $f_{B}(x, y)$ is not a function of $y$ alone, then for all $p_{X Y} \in \mathcal{P}_{f_{A} f_{B}}$, we have $\operatorname{supp}\left(p_{X Y}\right) \neq \mathcal{X} \times \mathcal{Y}$. Such $p_{X Y}$ can only lie on the boundary of the probability simplex $\Delta(\mathcal{X} \times \mathcal{Y})$.

Evaluating $R_{\text {sum }, t}^{A}$ is equivalent to evaluating the rate reduction $\rho_{t}^{A}$. Notice, however, that in (3), all the auxiliary random variables appear only as conditioned random variables whereas this is not the case in (1). As discussed in

Remark 7 in Section III-D this difference is critical as it enables us to characterize $\rho_{\infty}:=\lim _{t \rightarrow \infty} \rho_{t}^{A}=\lim _{t \rightarrow \infty} \rho_{t}^{B}$ which then gives us a characterization of $R_{\text {sum }, \infty}$ as $R_{\text {sum }, \infty}=H(X \mid Y)+H(Y \mid X)-\rho_{\infty}$. The rate reduction functional is the key to the characterization.

\section{B. Characterization of $\rho_{\infty}$ for special families of joint source pmfs}

Generally speaking, $R_{\text {sum }, t}^{A}, \rho_{t}^{A}, R_{\text {sum }, 0}$ and $\rho_{\infty}$ are functionals of $p_{X Y}, f_{A}$, and $f_{B}$. We will view $R_{\text {sum }, t}^{A}\left(p_{X Y}\right)$, $\rho_{t}^{A}\left(p_{X Y}\right), R_{\text {sum }, \infty}\left(p_{X Y}\right)$ and $\rho_{\infty}\left(p_{X Y}\right)$ as functionals of $p_{X Y}$ with $f_{A}$ and $f_{B}$ fixed in order to emphasize the dependence on $p_{X Y}$. Ideally, we would like to characterize and evaluate $\rho_{\infty}\left(p_{X Y}\right)$ for a single specified joint source pmf $p_{X Y}$ as in the point-to-point and Wyner-Ziv rate-distortion functions. As will become clear in the sequel, it is easier to characterize $\rho_{\infty}$ for a whole family of joint source pmfs $\mathcal{P}_{X Y}$ that is closed (in a sense that will be made precise in Section [II-C) than for a single joint source pmf $p_{X Y}$. In this section, we will state and discuss the 
characterization of $\rho_{\infty}$ for two special but important families of joint source pmfs namely, the family of all product pmfs $\mathcal{P}_{X Y}=\left\{p_{X} p_{Y} \mid p_{X} \in \Delta(\mathcal{X}), p_{Y} \in \Delta(\mathcal{Y})\right\}$ (cf. Proposition 1) and the family of all joint source pmfs $\mathcal{P}_{X Y}=\Delta(\mathcal{X} \times \mathcal{Y})$ (cf. Proposition 2). These propositions are special cases of a general result contained in Theorem 1 that will be stated and proved in Section III-D These propositions are intended to help understand the general result to follow without the additional complexity.

We begin by stating the characterization of the infinite-message limit for the family of all independent sources. This simplifies the characterization because independent sources are completely specified by the marginal distributions $p_{X}$ and $p_{Y}$.

Proposition 1: Let $\mathcal{F}_{1}$ be the set of functionals $\rho:\left\{p_{X} p_{Y} \mid p_{X} \in \Delta(\mathcal{X}), p_{Y} \in \Delta(\mathcal{Y})\right\} \rightarrow \mathbb{R}$ satisfying the following three conditions:

1) $\rho_{0}$-majorization: $\forall p_{X Y} \in \mathcal{P}_{X Y}, \rho\left(p_{X Y}\right) \geq \rho_{0}\left(p_{X Y}\right)$.

2) Concavity with respect to $p_{X}$ given $p_{Y}: \forall p_{Y}, \rho\left(p_{X} p_{Y}\right)$ is concave with respect to $p_{X}$.

3) Concavity with respect to $p_{Y}$ given $p_{X}: \forall p_{X}, \rho\left(p_{X} p_{Y}\right)$ is concave with respect to $p_{Y}$.

Then the functional $\rho_{\infty}$ is the least element of the set $\mathcal{F}_{1}$ with majorization as the partial ordering relation, i.e., (i) $\rho_{\infty} \in \mathcal{F}_{1}$, (ii) for all $\rho \in \mathcal{F}_{1}$ and all $p_{X}, p_{Y}$, we have $\rho_{\infty}\left(p_{X} p_{Y}\right) \leq \rho\left(p_{X} p_{Y}\right)$.

The set of functionals $\mathcal{F}_{1}$ is partially ordered with respect to majorization. In general, a partially ordered set may not have a least element. Proposition 1 asserts that $\mathcal{F}_{1}$ has a least element and that it is precisely $\rho_{\infty}$. When a least element exists, it is necessarily unique.

Proposition 1 provides a limit-free characterization of $\rho_{\infty}$ in that there is no parameter $t$ which needs to be sent to infinity. Unlike in Fact 1, this characterization is free of auxiliary random variables and their associated conditional entropy constraints, Markov chains, and cardinality bounds. In Fact 1, the dependency of the minimum sum-rate on the desired functions is captured only through the conditional entropy constraints $H\left(f_{A}(X, Y) \mid X, U^{t}\right)=$ $H\left(f_{B}(X, Y) \mid Y, U^{t}\right)=0$. These constraints depend on not only the joint source pmf $p_{X Y}$ but also on the auxiliary random variables $U^{t}$. In contrast, in Proposition 1, this dependency is captured by the simpler constraints $H\left(f_{A}(X, Y) \mid X\right)=$ $H\left(f_{B}(X, Y) \mid Y\right)=0$ which appear as part of the definition of $\mathcal{P}_{f_{A} f_{B}}$ or equivalently $\rho_{0}$. These simpler constraints are completely free of the auxiliary random variables $U^{t}$ and can be directly checked for any given $p_{X Y}$. If $f_{A}$ and $f_{B}$ are changed, then $\mathcal{P}_{f_{A} f_{B}}$ is changed. This changes $\rho_{0}$ which, in turn, changes $\mathcal{F}_{1}$ and therefore $\rho_{\infty}$. The characterization in Proposition 1 is implicit and nonconstructive because it does not directly provide an algorithm for finding the least element $\rho_{\infty}$ of $\mathcal{F}_{1}$. However, we shall see that the corollaries to Theorem 1 in Section $\amalg$ II-D lead to a simple optimality test for any achievable sum-rate functional and an iterative algorithm for evaluating $\rho_{\infty}$ (cf. Section IV).

The infinite-message rate-reduction functional for independent sources turned out to be concave with respect to $p_{X}$ for each fixed $p_{Y}$ and also concave with respect to $p_{Y}$ for each fixed $p_{X}$. In extending this characterization to $\Delta(X \times Y)$, the family of all joint source pmfs, some additional qualifications are needed. Specifically, the concavity with respect to the $X$-marginal distribution $p_{X}$ holds for each fixed conditional distribution $p_{Y \mid X}$ as opposed to each fixed $Y$-marginal distribution $p_{Y}$. Likewise, concavity with respect to the $Y$-marginal $p_{Y}$ holds for each fixed conditional $p_{X \mid Y}$ as opposed to each fixed $X$-marginal. The counterpart of Proposition 1 for $\Delta(X \times Y)$ is given by the following proposition. 
Proposition 2: Let $\mathcal{F}_{2}$ be the set of functionals $\rho: \Delta(\mathcal{X} \times \mathcal{Y}) \rightarrow \mathbb{R}$ satisfying the following three conditions:

1) $\rho_{0}$-majorization: $\forall p_{X Y} \in \Delta(\mathcal{X} \times \mathcal{Y}), \rho\left(p_{X Y}\right) \geq \rho_{0}\left(p_{X Y}\right)$.

2) Concavity with respect to $p_{X}$ given $p_{Y \mid X}: \forall p_{Y \mid X}, \rho\left(p_{Y \mid X} p_{X}\right)$ is concave with respect to $p_{X}$.

3) Concavity with respect to $p_{Y}$ given $p_{X \mid Y}: \forall p_{X \mid Y}, \rho\left(p_{X \mid Y} p_{Y}\right)$ is concave with respect to $p_{Y}$.

Then the functional $\rho_{\infty}$ is the least element of the set $\mathcal{F}_{2}$ with majorization as the partial ordering relation, i.e., (i) $\rho_{\infty} \in \mathcal{F}_{2}$, (ii) for all $\rho \in \mathcal{F}_{2}$ and all $p_{X Y} \in \Delta(\mathcal{X} \times \mathcal{Y})$, we have $\rho_{\infty}\left(p_{X Y}\right) \leq \rho\left(p_{X Y}\right)$.

Observing the similarity of the characterizations in Propositions 1 and 2 it is natural to wonder whether there are other families of joint source pmfs for which similar characterizations hold. In other words, can the results of these propositions be unified and generalized in some way? What are the key properties required of a family of joint source pmfs for such a characterization to hold? This is an important question because one would like to have a characterization for as small a family as possible, ideally, for a single specified joint source pmf. To answer this question, we must first examine the key properties that are shared by the family of all product pmfs and the family of all joint pmfs. We will do this in the next subsection and then state and prove the general result in Section $\amalg I-D$

\section{Marginal-perturbations-closed family of joint pmfs $\mathcal{P}_{X Y}$}

The family of all product pmfs and the family of all pmfs share the following common property: if a joint pmf belongs to the family, then so does any other joint pmf that shares the same conditional pmf. Put another way (and made precise below), these families of joint pmfs are closed with respect to marginal perturbations. This type of closedness property is needed for the concavity conditions 2) and 3) of Propositions 1 and 2 to even make sense. The notion of marginal perturbations is made precise in the following definition.

Definition 1: (X-marginal and $Y$-marginal perturbation sets $\mathcal{P}_{Y \mid X}\left(p_{X Y}\right)$ and $\left.\mathcal{P}_{X \mid Y}\left(p_{X Y}\right)\right)$ The set of $X$-marginal perturbations of a pmf $p_{X Y} \in \Delta(X \times Y)$ is defined as

$$
\mathcal{P}_{Y \mid X}\left(p_{X Y}\right):=\left\{p_{X Y}^{\prime} \in \Delta(\mathcal{X} \times \mathcal{Y}): p_{X Y}^{\prime} \ll p_{X Y}, p_{X Y}^{\prime} p_{X}=p_{X Y} p_{X}^{\prime}\right\}
$$

where $p_{X}$ and $p_{X}^{\prime}$ denote the $X$-marginals of $p_{X Y}$ and $p_{X Y}^{\prime}$ respectively. Similarly, let

$$
\mathcal{P}_{X \mid Y}\left(p_{X Y}\right):=\left\{p_{X Y}^{\prime} \in \Delta(\mathcal{X} \times \mathcal{Y}): p_{X Y}^{\prime} \ll p_{X Y}, p_{X Y}^{\prime} p_{Y}=p_{X Y} p_{Y}^{\prime}\right\}
$$

denote the set of $Y$-marginal perturbations of $p_{X Y}$ where $p_{Y}$ and $p_{Y}^{\prime}$ denote the $Y$-marginals of $p_{X Y}$ and $p_{X Y}^{\prime}$ respectively.

Essentially, $\mathcal{P}_{Y \mid X}\left(p_{X Y}\right)$ is the collection of all joint pmfs $p_{X Y}^{\prime}$ which have the same conditional pmf $p_{Y \mid X}$, that is, $p_{X Y}^{\prime}=p_{Y \mid X} \cdot p_{X}^{\prime}$ on $\operatorname{supp}\left(p_{X Y}^{\prime}\right)$. The subtlety is that the conditional pmf $p_{Y \mid X}^{\prime}$ of the joint pmf $p_{X Y}^{\prime}$ is well-defined only on $\operatorname{supp}\left(p_{X}^{\prime}\right) \times \mathcal{Y}$. Corresponding statements can be made for $\mathcal{P}_{X \mid Y}\left(p_{X Y}\right)$. The sets $\mathcal{P}_{Y \mid X}\left(p_{X Y}\right)$ and $\mathcal{P}_{X \mid Y}\left(p_{X Y}\right)$ are nonempty as they contain $p_{X Y}$.

Remark 2: For all $p_{X Y}$ : (i) $\mathcal{P}_{Y \mid X}\left(p_{X Y}\right)$ and $\mathcal{P}_{X \mid Y}\left(p_{X Y}\right)$ are convex sets of joint pmfs; (ii) if $p_{X Y}^{\prime} \in \mathcal{P}_{Y \mid X}\left(p_{X Y}\right)$ then $\mathcal{P}_{Y \mid X}\left(p_{X Y}^{\prime}\right) \subseteq \mathcal{P}_{Y \mid X}\left(p_{X Y}\right)$; and (iii) if $p_{X Y}^{\prime} \in \mathcal{P}_{X \mid Y}\left(p_{X Y}\right)$ then $\mathcal{P}_{X \mid Y}\left(p_{X Y}^{\prime}\right) \subseteq \mathcal{P}_{X \mid Y}\left(p_{X Y}\right)$.

The family of joint pmfs $\mathcal{P}_{X Y}$ which is closed with respect to $X$-marginal and $Y$-marginal perturbations can be formally described as follows.

Definition 2: (Marginal-perturbations-closed family of joint pmfs $\left.\mathcal{P}_{X Y}\right)$ A family of joint pmfs $\mathcal{P}_{X Y} \subseteq \Delta(\mathcal{X} \times \mathcal{Y})$ will be called marginal-perturbations-closed if for all $p_{X Y} \in \mathcal{P}_{X Y}, \mathcal{P}_{Y \mid X}\left(p_{X Y}\right) \cup \mathcal{P}_{X \mid Y}\left(p_{X Y}\right) \subseteq \mathcal{P}_{X Y}$. 
The family of all product pmfs and the family of all pmfs are examples of marginal-perturbations-closed families of joint pmfs. Another example is the set of all joint pmfs with supports contained in a specified subset of $\mathcal{X} \times \mathcal{Y}$, i.e., $\mathcal{P}_{X Y}=\Delta(S)$ where $S \subseteq \mathcal{X} \times \mathcal{Y}$. If $q_{X} q_{Y}$ belongs to any marginal-perturbations-closed family with $\operatorname{supp}\left(q_{X}\right)=\mathcal{X}$ and $\operatorname{supp}\left(q_{Y}\right)=\mathcal{Y}$, then the family will also contain $\Delta(\mathcal{X}) \times \Delta(\mathcal{Y})$, that is, all product pmfs on $\mathcal{X} \times \mathcal{Y}$.

The characterizations in Propositions 1 and 2 can be generalized to all marginal-perturbations-closed families of joint pmfs. The statement and proof of this general result and related corollaries is the subject matter of the next subsection.

\section{Main result}

In Propositions 1 and 2 we introduced two families of functionals $\mathcal{F}_{1}$ and $\mathcal{F}_{2}$. They can be unified as follows.

Definition 3: (Marginal-perturbations-concave, $\rho_{0}$-majorizing family of functionals $\mathcal{F}\left(\mathcal{P}_{X Y}\right)$ ) Let $\mathcal{P}_{X Y}$ be any marginal-perturbations-closed family of joint source pmfs on $\Delta(\mathcal{X} \times \mathcal{Y})$. The set of marginal-perturbations-concave, $\rho_{0}$-majorizing family of functionals $\mathcal{F}\left(\mathcal{P}_{X Y}\right)$ is the set of all the functionals $\rho: \mathcal{P}_{X Y} \rightarrow \mathbb{R}$ satisfying the following three conditions:

1) $\rho_{0}$-majorization: $\forall p_{X Y} \in \mathcal{P}_{X Y}, \rho\left(p_{X Y}\right) \geq \rho_{0}\left(p_{X Y}\right)$.

2) Concavity with respect to $X$-marginal perturbations: $\forall p_{X Y} \in \mathcal{P}_{X Y}, \rho$ is concave on $\mathcal{P}_{Y \mid X}\left(p_{X Y}\right)$.

3) Concavity with respect to $Y$-marginal perturbations: $\forall p_{X Y} \in \mathcal{P}_{X Y}, \rho$ is concave on $\mathcal{P}_{X \mid Y}\left(p_{X Y}\right)$.

Remark 3: Since $\rho_{0}\left(p_{X Y}\right)=-\infty$ for all $p_{X Y} \notin \mathcal{P}_{f_{A} f_{B}}$, condition 1) of Definition 3 is trivially satisfied for all $p_{X Y} \in \mathcal{P}_{X Y} \backslash \mathcal{P}_{f_{A} f_{B}}$ (we use the convention that $\forall a \in \mathbb{R}, a>-\infty$ ). Thus the statement that $\rho$ majorizes $\rho_{0}$ on the set $\mathcal{P}_{X Y}$ is equivalent to the statement that $\rho$ majorizes $H(X \mid Y)+H(Y \mid X)$ on the set $\mathcal{P}_{f_{A} f_{B}} \cap \mathcal{P}_{X Y}$.

Remark 4: Conditions 2) and 3) do not imply that $\rho$ is concave on $\mathcal{P}_{X Y}$. In fact, $\mathcal{P}_{X Y}$ itself may not be convex. For example, the set $\mathcal{P}_{X Y}=\left\{p_{X} p_{Y} \mid p_{X} \in \Delta(\mathcal{X}), p_{Y} \in \Delta(\mathcal{Y})\right\}$ is not convex.

We now state and prove the main result of this paper.

Theorem 1: The functional $\rho_{\infty}$ is the least element of the set $\mathcal{F}\left(\mathcal{P}_{X Y}\right)$ with majorization as the partial ordering relation, i.e., (i) $\rho_{\infty} \in \mathcal{F}\left(\mathcal{P}_{X Y}\right)$, (ii) for all $\rho \in \mathcal{F}\left(\mathcal{P}_{X Y}\right)$ and all $p_{X Y} \in \mathcal{P}_{X Y}$, we have $\rho_{\infty}\left(p_{X Y}\right) \leq \rho\left(p_{X Y}\right)$.

This theorem provides a limit-free characterization of $R_{\text {sum, } \infty}$ for any marginal-perturbations-closed family of pmfs. In terms of the optimal coding strategy, $R_{\text {sum }, \infty}$ can be approached by a sequence of "Wyner-Ziv like" codes, just as in the achievability proof of Fact 1 . This is illustrated in Appendix C for the example studied in Section $\mathrm{V}-\mathrm{B}$

Proof: To prove Theorem 1 we will use a Lemma that establishes a connection between a $t$-message interactive coding problem and several related $(t-1)$-message interactive coding subproblems. Intuitively, to construct a $t$ message interactive code with initial terminal $A$, we need to begin by choosing the first message. This corresponds to choosing the auxiliary random variable $U_{1}$. Then for each realization $U_{1}=u_{1}$, constructing the remaining part of the code becomes a $(t-1)$-message subproblem with initial terminal $B$ with the same desired functions, but with a different source $\operatorname{pmf} p_{X Y \mid U_{1}}\left(\cdot, \cdot \mid u_{1}\right) \in \mathcal{P}_{Y \mid X}\left(p_{X Y}\right)$. Lemma 1 connects the rate reduction of the original problem to the rate reduction of the subproblems. We can repeat this procedure recursively to construct a $(t-1)$-message interactive code with initial terminal $B$. After $t$ steps of recursion, we will be left with the trivial 0 -message problem. 
Lemma 1: (i) For all $t \in \mathbb{Z}^{+}$and all $p_{X Y} \in \mathcal{P}_{X Y}$,

$$
\rho_{t}^{A}\left(p_{X Y}\right)=\max _{p_{U_{1} \mid X}}\left\{\sum_{u_{1} \in \operatorname{supp}\left(p_{U_{1}}\right)} p_{U_{1}}\left(u_{1}\right) \rho_{t-1}^{B}\left(p_{X Y \mid U_{1}}\left(\cdot, \cdot \mid u_{1}\right)\right)\right\} .
$$

(ii) For all $t \in \mathbb{Z}^{+}$and all $q_{X Y} \in \mathcal{P}_{X Y}, \rho_{t}^{A}$ is concave on $\mathcal{P}_{Y \mid X}\left(q_{X Y}\right)$.

(iii) For all $t \in \mathbb{Z}^{+}$and all $q_{X Y} \in \mathcal{P}_{X Y}$, if $\rho: \mathcal{P}_{X Y} \rightarrow \mathbb{R}$ is concave on $\mathcal{P}_{Y \mid X}\left(q_{X Y}\right)$ and if for all $p_{X Y} \in \mathcal{P}_{Y \mid X}\left(q_{X Y}\right)$, $\rho_{t-1}^{B}\left(p_{X Y}\right) \leq \rho\left(p_{X Y}\right)$, then for all $p_{X Y} \in \mathcal{P}_{Y \mid X}\left(q_{X Y}\right), \rho_{t}^{A}\left(p_{X Y}\right) \leq \rho\left(p_{X Y}\right)$.

(iv) The results of parts (i) - (iii) above also hold if $A$ is swapped with $B$ and simultaneously, $\mathcal{P}_{Y \mid X}$ and $p_{U_{1} \mid X}$ are replaced by $\mathcal{P}_{X \mid Y}$ and $p_{U_{1} \mid Y}$ respectively.

The proof of Lemma 1 is presented in Appendix $\mathrm{A}$ Here we will focus on explaining the intuition underlying the proof of the Lemma. Due to (3), the functional $\rho_{t}^{A}$ can be expressed as the maximum of $H\left(X \mid Y, U^{t}\right)+H\left(Y \mid X, U^{t}\right)$ where the maximum is over all choices of auxiliary random variables $U^{t}$. Then the following two properties can be established: (a) by conditioning on the random variable $U_{1}, H\left(X \mid Y, U^{t}\right)+H\left(Y \mid X, U^{t}\right)$ can be written as a convex combination of $\left\{H\left(X \mid Y, U_{2}^{t}, U_{1}=u_{1}\right)+H\left(Y \mid X, U_{2}^{t}, U_{1}=u_{1}\right)\right\}_{u_{1} \in \mathcal{U}_{1}}$; (b) for any fixed choice of $U_{1}$ and each realization $U_{1}=u_{1}$, the maximum of $H\left(X \mid Y, U_{2}^{t}, U_{1}=u_{1}\right)+H\left(Y \mid X, U_{2}^{t}, U_{1}=u_{1}\right)$ over all choices of $U_{2}^{t}$ is $\rho_{t-1}^{B}$, that is, the rate reduction of the $(t-1)$-message subproblem with initial terminal $B$. From (a) and (b) it would follow that for each fixed choice of $U_{1}, \rho_{t}^{A}$ is bounded from below by a convex combination of $\rho_{t-1}^{B}$. Moreover, for the optimal choice of $U_{1}$ this lower bound can be shown to be tight. This line of reasoning would establish part (i) of the lemma. Part (i) would, in turn, imply that $\rho_{t}^{A}$ is a concave functional (part (ii) of the lemma) and is the least concave functional that majorizes $\rho_{t-1}^{B}$ (part (iii) of the lemma).

We are now ready to prove Theorem 1

Proof of part (i) of Theorem [1: We need to verify that $\rho_{\infty}$ satisfies all three conditions in Definition 3 ,

1) Since $\forall p_{X Y} \in \mathcal{P}_{X Y}, R_{\text {sum }, \infty}\left(p_{X Y}\right) \leq R_{\text {sum }, 0}\left(p_{X Y}\right)$, we have $\rho_{\infty}\left(p_{X Y}\right) \geq \rho_{0}\left(p_{X Y}\right)$. Thus $\rho_{\infty}$ is $\rho_{0}$-majorizing.

2) Due to part (ii) of Lemma 1 for all $t \in \mathbb{Z}^{+}$and all $q_{X Y} \in \mathcal{P}_{X Y}, \rho_{t}^{A}$ is concave on $\mathcal{P}_{Y \mid X}\left(q_{X Y}\right)$. Since for all $q_{X Y} \in \mathcal{P}_{X Y}, \lim _{t \rightarrow \infty} \rho_{t}^{A}\left(q_{X Y}\right)=\rho_{\infty}\left(q_{X Y}\right)$, it follows that $\rho_{\infty}$ is concave on $\mathcal{P}_{Y \mid X}\left(q_{X Y}\right)$.

3) Due to parts (ii) and (iv) of Lemma 1 for all $t \in \mathbb{Z}^{+}$and all $q_{X Y} \in \mathcal{P}_{X Y}, \rho_{t}^{B}$ is concave on $\mathcal{P}_{X \mid Y}\left(q_{X Y}\right)$. Since for all $q_{X Y} \in \mathcal{P}_{X Y}, \lim _{t \rightarrow \infty} \rho_{t}^{B}\left(q_{X Y}\right)=\rho_{\infty}\left(q_{X Y}\right)$, it follows that $\rho_{\infty}$ is concave on $\mathcal{P}_{X \mid Y}\left(q_{X Y}\right)$.

Thus, $\rho_{\infty} \in \mathcal{F}\left(\mathcal{P}_{X Y}\right)$.

Proof of part (ii) of Theorem [1. It is sufficient to show that: $\forall \rho \in \mathcal{F}\left(\mathcal{P}_{X Y}\right), \forall p_{X Y} \in \mathcal{P}_{X Y}$, and $\forall t \in \mathbb{Z}^{+} \cup\{0\}$, $\rho_{t}^{A}\left(p_{X Y}\right) \leq \rho\left(p_{X Y}\right)$ and $\rho_{t}^{B}\left(p_{X Y}\right) \leq \rho\left(p_{X Y}\right)$. We prove this by induction on $t$. For $t=0$, the result is true by condition $1)$ in Definition 3. $\rho_{0}^{A}\left(p_{X Y}\right)=\rho_{0}^{B}\left(p_{X Y}\right)=\rho_{0}\left(p_{X Y}\right) \leq \rho\left(p_{X Y}\right)$. Now assume that for an arbitrary $t \in \mathbb{Z}^{+}, \rho_{t-1}^{A}\left(p_{X Y}\right) \leq$ $\rho\left(p_{X Y}\right)$ and $\rho_{t-1}^{B}\left(p_{X Y}\right) \leq \rho\left(p_{X Y}\right)$ hold. From parts (iii) and (iv) of Lemma 1, it follows that $\rho_{t}^{A}\left(p_{X Y}\right) \leq \rho\left(p_{X Y}\right)$ and $\rho_{t}^{B}\left(p_{X Y}\right) \leq \rho\left(p_{X Y}\right)$. This completes the proof of Theorem 1.

Remark 5: In the proof of Theorem 11 the marginal-perturbations-closed property of $\mathcal{P}_{X Y}$ is used in Remark 9 in Appendix A, which is in turn used in the proof of part (iii) of Lemma 1 .

Remark 6: It can be verified that the functional $(H(X \mid Y)+H(Y \mid X))$ belongs to $\mathcal{F}(\Delta(X \times \mathcal{Y}))$. Although both $(H(X \mid Y)+H(Y \mid X))$ and $\rho_{\infty}\left(p_{X Y}\right)$ are concave on $X$-marginal and $Y$-marginal perturbation sets of $p_{X Y}$, this alone does not guarantee the convexity or concavity of $R_{\text {sum }, \infty}\left(p_{X Y}\right)=(H(X \mid Y)+H(Y \mid X))-\rho_{\infty}\left(p_{X Y}\right)$ on the marginal perturbation sets of $p_{X Y}$. An independent argument, however, can be used to prove that $R_{\text {sum, } \infty}$ is concave: 
Proposition 3: (i) For all $t \in \mathbb{Z}^{+}, R_{\text {sum }, t}^{A}\left(p_{X Y}\right)$ is a concave functional of $p_{X Y}$ for $p_{X Y} \in \Delta(\mathcal{X} \times \mathcal{Y})$. (ii) $R_{\text {sum, } \infty}\left(p_{X Y}\right)$ is a concave functional of $p_{X Y}$ for $p_{X Y} \in \Delta(X \times Y)$.

The proof of this result is presented in Appendix B We note that the concavity in Proposition 3 is not with respect to marginal perturbations.

Remark 7: For each $t, \rho_{t}^{A}$ is the maximum of $\left(H\left(X \mid Y, U^{t}\right)+H\left(Y \mid X, U^{t}\right)\right)$, where $U^{t}$ appear only as conditioned random variables. This enables us to use the "law of total conditional entropy" (which corresponds to convexification) and arrive at (5]) (see Appendix (A). Notice, however, that $R_{\text {sum, }, \infty}$ is the minimum value of $\left(I\left(X ; U^{t} \mid Y\right)+I\left(Y ; U^{t} \mid X\right)\right)$ over all $U^{t}$ where $U^{t}$ do not appear as conditioned random variables. Therefore, we cannot use the same technique to express $R_{\text {sum }, t}^{A}$ as a convex combination of $R_{\text {sum }, t-1}^{B}$. Due to these reasons, although evaluating $\rho_{\infty}$ is equivalent to evaluating $R_{\text {sum }, \infty}$, the rate reduction functional is the key to the characterization as remarked in Section $\amalg I-\mathrm{A}$.

Lemma 1 implies that $\rho_{t}^{A}$ is the least functional that is concave on $X$-marginal perturbation sets and majorizes $\rho_{t-1}^{B}$, and $\rho_{t}^{B}$ is the least functional that is concave on $Y$-marginal perturbation sets and majorizes $\rho_{t-1}^{A}$. These implications lead to the following corollary.

Corollary 1: (Constructing $\rho_{t}^{A}$ and $\rho_{t}^{B}$ from $\rho_{t-1}^{B}$ and $\rho_{t-1}^{A}$ respectively) For all $t \in \mathbb{Z}^{+}$and all $p_{X Y} \in \mathcal{P}_{X Y}$, we have (i) $\operatorname{ch}\left(\operatorname{hypo}_{\mathcal{P}_{Y \mid X}\left(p_{X Y}\right)} \rho_{t-1}^{B}\right)=\operatorname{hypo}_{\mathcal{P}_{Y \mid X}\left(p_{X Y}\right)} \rho_{t}^{A}$ and (ii) $\operatorname{ch}\left(\operatorname{hypo}_{\mathcal{P}_{X \mid Y}\left(p_{X Y}\right)} \rho_{t-1}^{A}\right)=\operatorname{hypo}_{\mathcal{P}_{X \mid Y}\left(p_{X Y}\right)} \rho_{t}^{B}$.

In the convex optimization literature, $\left(-\rho_{1}^{A}\right)$ is also called the double Legendre-Fenchel transform or convex biconjugate of $\left(-\rho_{t-1}^{B}\right)$ [12]. Corollary 1 enables us to evaluate $\rho_{t}^{A}$ and $\rho_{t}^{B}$ for arbitrary $t$ using an iterative algorithm described in Section IV

Corollary 2 establishes a connection between the concavity property of $\rho_{t}^{A}$ (respectively $\rho_{t}^{B}$ ) and the optimality of $\rho_{t}^{A}$ (respectively $\rho_{t}^{B}$ ).

Corollary 2: (Concavity and optimality of $\rho_{t}^{A}$ and $\rho_{t}^{B}$ ) For all $t \in \mathbb{Z}^{+}$, the following three conditions are equivalent:

(i) $\forall p_{X Y} \in \mathcal{P}_{X Y}, \rho_{t}^{A}\left(p_{X Y}\right)=\rho_{\infty}\left(p_{X Y}\right)$,

(ii) $\forall p_{X Y} \in \mathcal{P}_{X Y}, \rho_{t}^{A}\left(p_{X Y}\right)=\rho_{t+1}^{B}\left(p_{X Y}\right)$,

(iii) $\forall p_{X Y} \in \mathcal{P}_{X Y}, \rho_{t}^{A}$ is concave on $\mathcal{P}_{X \mid Y}\left(p_{X Y}\right)$.

For all $t \in \mathbb{Z}^{+}$, the following three conditions are also equivalent:

(iv) $\forall p_{X Y} \in \mathcal{P}_{X Y}, \rho_{t}^{B}\left(p_{X Y}\right)=\rho_{\infty}\left(p_{X Y}\right)$,

(v) $\forall p_{X Y} \in \mathcal{P}_{X Y}, \rho_{t}^{B}\left(p_{X Y}\right)=\rho_{t+1}^{A}\left(p_{X Y}\right)$,

(vi) $\forall p_{X Y} \in \mathcal{P}_{X Y}, \rho_{t}^{B}$ is concave on $\mathcal{P}_{Y \mid X}\left(p_{X Y}\right)$.

Proof: We will prove the equivalence of conditions (i), (ii) and (iii). The proof of the equivalence of conditions (iv), (v), and (vi) is analogous. Condition (i) implies (ii) because $\rho_{t}^{A} \leq \rho_{t+1}^{B} \leq \rho_{\infty}$. Condition (ii) implies (iii) due to parts (ii) and (iv) of Lemma 1, Condition (iii) implies (i) due to the following reasons: From Lemma 1(ii), Condition (iii), and the fact that $\rho_{t}^{A} \geq \rho_{0}$, we have $\rho_{t}^{A} \in \mathcal{F}\left(\mathcal{P}_{X Y}\right)$. By Theorem $11 \rho_{t}^{A} \geq \rho_{\infty}$. But the inequality $\rho_{t}^{A} \leq \rho_{\infty}$ always holds. Therefore we have $\rho_{t}^{A}=\rho_{\infty}$.

Due to Lemma 1, for all $t \in \mathbb{Z}^{+}, \rho_{t}^{A}$ always satisfies conditions 1) and 2) in Definition 3 ( $\rho_{0}$-majorization and concavity with respect to $X$-marginal perturbations), but not necessarily condition 3 ); $\rho_{t}^{B}$ always satisfies conditions $1)$ and 3$)$ in Definition 3 ( $\rho_{0}$-majorization and concavity with respect to $Y$-marginal perturbations), but not necessarily condition 2). By Theorem $11 \rho_{\infty}$ satisfies all three conditions of Definition 3 and is not larger than any $\rho$ which satisfies all three conditions. By Corollary 2] once $\rho_{t}^{A}$ satisfies condition 3) in Definition 3, which is also condition 
(iii) in Corollary 2] then $\rho_{t}^{A}=\rho_{t+1}^{B}=\rho_{\infty}$. Similarly, once $\rho_{t}^{B}$ satisfies condition 2) in Definition 3, then $\rho_{t}^{B}=\rho_{t+1}^{A}=\rho_{\infty}$. Thus, $\rho_{t}^{A}$ and $\rho_{t}^{B}$ equal $\rho_{\infty}$ iff they satisfy all three conditions. If all three conditions are not satisfied (two are always satisfied), it is beneficial to increase the number of messages. For example, if $\rho_{t}^{A}$ is not concave on a $Y$-marginal perturbation set, then for some $p_{X Y}, \rho_{t}^{A}\left(p_{X Y}\right)<\rho_{t+1}^{B}\left(p_{X Y}\right)$ (and $\rho_{t+1}^{B}\left(p_{X Y}\right) \leq \rho_{t+2}^{A}\left(p_{X Y}\right)$ ) because otherwise $\rho_{t}^{A}=\rho_{t+1}^{B}$ and by Corollary $2, \rho_{t}^{A}=\rho_{\infty}$. In summary, the concavity of $\rho_{t}^{A}$ on $Y$-marginal perturbation sets is equivalent to the optimality of $\rho_{t}^{A}$ and the concavity of $\rho_{t}^{B}$ on $X$-marginal perturbation sets is equivalent to the optimality of $\rho_{t}^{B}$. Moreover, if a single additional message is not beneficial then no number of additional messages will be beneficial.

Since every $\rho \in \mathcal{F}\left(\mathcal{P}_{X Y}\right)$ gives an upper bound for $\rho_{\infty},(H(X \mid Y)+H(Y \mid X)-\rho)$ gives a lower bound for $R_{\text {sum }, \infty}$. This fact provides a simple method to test if an achievable sum-rate functional is optimal.

Corollary 3: (Optimality test for an achievable sum-rate) Let $R^{*}$ be a sum-rate functional which is achievable using an arbitrary number of messages. If $\rho^{*}:=\left(H(X \mid Y)+H(Y \mid X)-R^{*}\right) \in \mathcal{F}\left(\mathcal{P}_{X Y}\right)$, then $R^{*}=R_{\text {sum, }, \infty}$.

Proof: If $R^{*}$ is a sum-rate functional which is achievable then $\forall p_{X Y} \in \mathcal{P}_{X Y}, R^{*}\left(p_{X Y}\right) \geq R_{\text {sum, }}\left(p_{X Y}\right)$. If $\rho^{*} \in$ $\mathcal{F}\left(\mathcal{P}_{X Y}\right)$ then by Theorem $1, \rho^{*} \geq \rho_{\infty}$ and thus $R^{*} \leq R_{\text {sum }, \infty}$. Therefore $R^{*}=R_{\text {sum }, \infty}$.

By Corollary [3, in order to certify that an achievable sum-rate is optimal, we only need to verify majorization and concavity properties of the corresponding rate reduction functional. The nontrivial part of the test is to verify the concavity properties. We will demonstrate this test on two examples in Section $\nabla$

\section{ITERATIVE ALGORIthM FOR COMPUTING $R_{\text {sum }, t}^{A}(\cdot)$ AND $R_{\text {sum }, \infty}(\cdot)$}

Although Theorem 1 provides a characterization of $\rho_{\infty}$ and $R_{\text {sum }, \infty}$ that is not obtained by taking a limit, it does not directly provide an algorithm to evaluate $R_{\text {sum }, \infty}$. If an expression for an achievable sum-rate as a function of $p_{X Y}$ is available, we can use Corollary 3 to test whether it coincides with the infinite-message limit. In some cases, as in Section V] we may get lucky and the achievable sum-rate expression will pass the test. In such cases, we will obtain a closed-form expression for the infinite-message limit. In other cases, a computer-based numerical evaluation may be the only recourse. To efficiently represent and search for the least element of $\mathcal{F}\left(\mathcal{P}_{X Y}\right)$ is nontrivial because each element is a functional; not a scalar. Corollary 1 however, inspires an iterative algorithm for evaluating $R_{\text {sum,t }}^{A}$ and $R_{\text {sum }, \infty}$. Corollary 1 states that $\rho_{t}^{A}$ can be constructed on any given $X$-marginal perturbation set by taking the convex hull of the hypograph of $\rho_{t-1}^{B}$ on the $X$-marginal perturbation set. To determine $\rho_{t}^{A}\left(p_{X Y}\right)$ for all $p_{X Y} \in \mathcal{P}_{X Y}$, we can, in principle, first choose a cover for $\mathcal{P}_{X Y}$ made up of $X$-marginal perturbation sets, say $\left\{\mathcal{P}_{Y \mid X}\left(p_{X Y}\right)\right\}_{p_{X Y} \in \mathcal{A}}$, where $\mathcal{A} \subseteq \mathcal{P}_{X Y}$, and then take convex hulls in every $X$-marginal perturbation set in the cover. This idea leads to the following "alternating marginal concavification" algorithm.

\section{Algorithm to evaluate $R_{\text {sum }, t}^{A}$ and $R_{\text {sum }, t}^{B}$}

- Initialization: Choose a marginal-perturbations-closed family $\mathcal{P}_{X Y}$ containing all joint source pmfs of interest. Define $\rho_{0}^{A}\left(p_{X Y}\right)=\rho_{0}^{B}\left(p_{X Y}\right)=\rho_{0}\left(p_{X Y}\right)$ by equation (4) in the domain $\mathcal{P}_{X Y}$. Choose a cover for $\mathcal{P}_{X Y}$ made up of $X$-marginal perturbation sets, denoted by $\left\{\mathcal{P}_{Y \mid X}\left(p_{X Y}\right)\right\}_{p_{X Y} \in \mathcal{A}}$, where $\mathcal{A} \subseteq \mathcal{P}_{X Y}$. Also choose a cover for $\mathcal{P}_{X Y}$ made up of $Y$-marginal perturbation sets, denoted by $\left\{\mathcal{P}_{X \mid Y}\left(p_{X Y}\right)\right\}_{p_{X Y} \in \mathcal{B}}$, where $\mathcal{B} \subseteq \mathcal{P}_{X Y}$.

- Loop: For $\tau=1$ through $t$ do the following:

For every $p_{X Y} \in \mathcal{A}$, do the following in the set $\mathcal{P}_{Y \mid X}\left(p_{X Y}\right)$ :

- Construct hypo $\mathcal{P}_{Y \mid X}\left(p_{X Y}\right) \rho_{\tau-1}^{B}$.

- Let $\rho_{\tau}^{A}$ be the upper boundary of $\operatorname{ch}\left(\operatorname{hypo}_{\mathcal{P}_{Y \mid X}\left(p_{X Y}\right)} \rho_{\tau-1}^{B}\right)$. 
For every $p_{X Y} \in \mathcal{B}$, do the following in the set $\mathcal{P}_{X \mid Y}\left(p_{X Y}\right)$ :

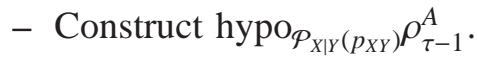

- Let $\rho_{\tau}^{B}$ be the upper boundary of $\operatorname{ch}\left(\operatorname{hypo}_{\mathcal{P}_{X \mid Y}\left(p_{X Y}\right)} \rho_{\tau-1}^{A}\right)$.

Optimality test:

if $\forall p_{X Y} \in \mathcal{P}_{X Y}, \rho_{\tau}^{A}\left(p_{X Y}\right)=\rho_{\tau-1}^{B}\left(p_{X Y}\right)$, then set $\rho_{t}^{A}=\rho_{t}^{B}=\rho_{\tau}^{A}$ and exit the loop.

if $\forall p_{X Y} \in \mathcal{P}_{X Y}, \rho_{\tau}^{B}\left(p_{X Y}\right)=\rho_{\tau-1}^{A}\left(p_{X Y}\right)$, then set $\rho_{t}^{A}=\rho_{t}^{B}=\rho_{\tau}^{B}$ and exit the loop.

- Output: $R_{\text {sum }, t}^{A}\left(p_{X Y}\right)=H(X \mid Y)+H(Y \mid X)-\rho_{t}^{A}\left(p_{X Y}\right)$ and $R_{\text {sum }, t}^{B}\left(p_{X Y}\right)=H(X \mid Y)+H(Y \mid X)-\rho_{t}^{B}\left(p_{X Y}\right)$.

To make computer-based numerical evaluation feasible, $\mathcal{P}_{X Y}$ has to be discretized. Once discretized, however, in each iteration, the amount of computation is the same and is fixed by the discretization step-size. Also note that results from each iteration are re-used in the following one. Therefore, for large $t$, the complexity to compute $R_{\text {sum }, t}^{A}$ grows linearly with respect to $t$.

$R_{\text {sum }, \infty}$ can also be evaluated to any precision, in principle, by running this iterative algorithm for $t=1,2, \ldots$, until some stopping criterion is met, e.g., the maximum difference between $\rho_{t-1}^{A}$ and $\rho_{t}^{A}$ on $\mathcal{P}_{X Y}$ falls below some threshold. Developing stopping criteria with precision guarantees requires some knowledge of the rate of convergence which is not established in this paper; the rate may, however, be empirically estimated. For the example presented in Section $\mathrm{V}-\mathrm{B}$, the empirical rate of convergence and the impact of the discretization step-size on the iterative evaluation is discussed. When the objective is to evaluate $R_{\text {sum }, \infty}\left(p_{X Y}\right)$ for all pmfs in $\mathcal{P}_{X Y}$, this iterative algorithm is much more efficient than using (1) to solve for $R_{\text {sum }, t}^{A}$ for each $p_{X Y}$ for $t=1,2, \ldots$, an approach which literally follows the definition of $R_{\text {sum }, \infty}$ as the limit of $R_{\text {sum }, t}^{A}$ as $t \rightarrow \infty$. Our iterative algorithm is based on Theorem 1 which is a limit-free characterization of $R_{\text {sum }, \infty}$.

\section{EXAMPLES}

For samplewise computation of the Boolean AND function of independent Bernoulli sources at both terminals, an achievable sum-rate functional was derived in closed analytic form in a previous work. Using Corollary 3 in Section $\mathrm{V}$-A we will show that this sum-rate functional is, in fact, optimal. In Section $\mathrm{V}$-B we will present and derive a closed-form analytic expression for an achievable sum-rate functional for another closely related problem wherein the Boolean AND function of independent Bernoulli sources is required to be computed at only one terminal. This coding strategy is based on a sequence of Wyner-Ziv codes (with random coding and binning) and uses, in the limit, an infinite number of infinitesimal-rate messages. We will also establish its optimality providing another illustration of the use of the optimality test in Corollary 3 . For this example, we will also illustrate a numerical implementation of the iterative algorithm described in Section IV

\section{A. $R_{\text {sum }, \infty}$ for independent binary sources and the Boolean AND function computed at both terminals}

In [1, Sec. IV.F], we studied the samplewise computation of the Boolean AND function at both terminals for independent Bernoulli sources, i.e., $\mathcal{X}=\mathcal{Y}=\{0,1\}, X \Perp Y, X \sim \operatorname{Ber}(p), Y \sim \operatorname{Ber}(q)$, and $f_{A}(x, y)=f_{B}(x, y)=x \wedge y$. An interesting interactive coding scheme was described in [1] where the individual rate for each message vanished as the number of messages went to infinity. The (achievable) infinite-message sum-rate of this scheme, denoted by $R^{*}$, was evaluated in closed form as

$$
R^{*}(p, q)= \begin{cases}h_{2}(p)+p h_{2}(q)+p \log _{2} q+p(1-q) \log _{2} e, & \text { if } 0 \leq p \leq q \leq 1, \\ R^{*}(q, p), & \text { if } 0 \leq q \leq p \leq 1 .\end{cases}
$$


Actually, the expression for $R^{*}(p, q)$ in (6) was only derived for the case $0<p \leq q<1$ in [1, Sec. IV.F]. From this, the expression for $R^{*}(p, q)$ for the case $0<q \leq p<1$ follows immediately by interchanging $p$ and $q$. If $p q=0$ then the samplewise Boolean AND function is always 0 and no message needs to be sent from any terminal. Thus for all $p, q \in[0,1], R^{*}(p, 0)=R^{*}(0, q)=0$. If $(1-p)(1-q)=0$ then either $p=1$, in which case all samples of the $X$-source are equal to one and the samples of the Boolean AND function coincide with the samples of the $Y$-source, or $q=1$, in which case the samples of the Boolean AND function coincide with those of the $X$-source. If $p=1$ then a single message from $B$ to $A$ of rate $h_{2}(q)$ would be sufficient to compute the samplewise Boolean AND function at both terminals and if $q=1$ then a single message from $A$ to $B$ of rate $h_{2}(p)$ would suffice.

Since $R^{*}(p, q)$ is an achievable sum-rate, $R^{*} \geq R_{\text {sum }, \infty}$. Using Theorem 1 we shall now prove that $R^{*}$ is, in fact, equal to $R_{\text {sum }, \infty}$. We will verify that $\rho^{*}:=H(X \mid Y)+H(Y \mid X)-R^{*}$ belongs to $\mathcal{F}\left(\mathcal{P}_{X Y}\right)$ for the product pmf family $\mathcal{P}_{X Y}$, which will imply, by Theorem 1(ii), that $\rho^{*} \geq \rho_{\infty}$, i.e., $R^{*} \leq R_{\text {sum }, \infty}$. We note that $R_{\text {sum }, \infty}$ is not evaluated using Theorem 1 Only part (ii) of Theorem 1 is used as a converse proof to show that the achievable sum-rate $R^{*}$ is $R_{\text {sum }, \infty}$.

Since the sources are independent, we take the marginal-perturbations-closed family to be $\mathcal{P}_{X Y}=\left\{p_{X} p_{Y} \mid p_{X} \in\right.$ $\left.\Delta(\mathcal{X}), p_{Y} \in \Delta(\mathcal{Y})\right\}$. For each product pmf $p_{X} p_{Y}$, the $X$-marginal and $Y$-marginal perturbation sets are $\mathcal{P}_{Y \mid X}\left(p_{X} p_{Y}\right)=$ $\left\{p_{X}^{\prime} p_{Y}: p_{X}^{\prime} \ll p_{X}\right\}$ and $\mathcal{P}_{X \mid Y}\left(p_{X} p_{Y}\right)=\left\{p_{X} p_{Y}^{\prime}: p_{Y}^{\prime} \ll p_{Y}\right\}$ respectively.

Parametric representation of joint source pmfs: Since both sources are binary, we can use the scalars $p=p_{X}(1)$ and $q=p_{Y}(1)$ to represent the marginal pmfs $p_{X}$ and $p_{Y}$ respectively. The product pmf $p_{X} p_{Y}$ can be represented by a point $(p, q) \in[0,1]^{2}$. In the remainder of this section and in Section $\mathrm{V}$-B for convenience we shall abuse notation and write $R_{\text {sum }, t}^{A}(p, q) \rho_{\text {sum }, t}^{A}(p, q), R_{\text {sum }, t}^{B}(p, q), \rho_{\text {sum }, t}^{B}(p, q)$, etc., instead of $R_{\text {sum }, t}^{A}\left(p_{X} p_{Y}\right) \rho_{\text {sum }, t}^{A}\left(p_{X} p_{Y}\right), R_{\text {sum }, t}^{B}\left(p_{X} p_{Y}\right)$, $\rho_{\text {sum }, t}^{B}\left(p_{X} p_{Y}\right)$, etc.

In this parametric representation, for all pmfs $(p, q) \in(0,1)^{2}$, the $X$-marginal and $Y$-marginal perturbation sets are the line segments $[0,1] \times\{q\}$ and $\{p\} \times[0,1]$ respectively. For all pmfs $(0, q)$, where $q \in(0,1)$, the $X$-marginal and $Y$-marginal perturbation sets are represented by $(0, q)$ and $\{0\} \times[0,1]$ respectively. For the pmf $(0,0)$, both the $X$-marginal and $Y$-marginal perturbation sets are $(0,0)$. The marginal perturbation sets of remaining pmfs $(p, q)$ on the boundary of $[0,1]^{2}$ can be derived by exploiting the symmetry in the problem (swap $p$ and $q$; then swap symbols 0 and 1 ).

It is straightforward to confirm that

$$
R_{\text {sum }, 0}(p, q)=\left\{\begin{array}{cc}
0, & \text { if }(p, q) \in \mathcal{P}_{f_{A} f_{B}}, \\
+\infty, & \text { otherwise, }
\end{array}\right.
$$

where $\mathcal{P}_{f_{A} f_{B}}=\{(p, q): p=0$ or $q=0$ or $p=q=1\}$. It is also straightforward to verify that for all $(p, q)$, $R^{*}(p, q) \leq R_{\text {sum }, 0}(p, q)=0$, or equivalently, $\rho^{*}(p, q) \geq \rho_{0}(p, q)$. By taking the first and second-order partial derivatives of $\rho^{*}(p, q)=h_{2}(p)+h_{2}(q)-R^{*}(p, q)$ with respect to $p$ and $q$, we can verify that for any fixed $q, \rho^{*}(p, q)$ is concave with respect to $p$ (and therefore also with respect to $p_{X}$ ), and for any fixed $p, \rho^{*}(p, q)$ is concave with respect to $q$ (and therefore also with respect to $p_{Y}$ ). Therefore, $\rho^{*}(p, q)$ is concave in every $X$-marginal and $Y$-marginal perturbation set. Therefore, $\rho^{*}(p, q) \in \mathcal{F}\left(\mathcal{P}_{X Y}\right)$. From Corollary 3 it follows that $R_{\text {sum }, \infty}(p, q)=R^{*}(p, q)$.

\section{B. $R_{\text {sum }, \infty}$ for independent binary sources and Boolean AND function computed at only terminal B}

Consider the problem in Section $\mathrm{V}-\mathrm{A}$ with one modification: the Boolean AND function needs to be computed only at terminal $B$, i.e., $f_{A}(x, y)=0$ and $f_{B}(x, y)=x \wedge y$. The joint source distribution is unchanged: $X \Perp Y$, 
$X \sim \operatorname{Ber}(p), Y \sim \operatorname{Ber}(q)$. The following sum-rate $R^{*}(p, q)$ is shown to be achievable in Appendix $\mathrm{C}$ using the technique that was developed in [1, Sec. IV.F].

$$
R^{*}(p, q)= \begin{cases}h_{2}(p)+p h_{2}(q)+p \log _{2} q+p(1-2 q) \log _{2} e, & \text { if } 0 \leq p \leq q \leq 1 / 2, \\ R^{*}(q, p), & \text { if } 0 \leq q \leq p \leq 1 / 2, \\ R^{*}(1-p, q), & \text { if } 0 \leq q \leq 1 / 2 \leq p \leq 1, \\ h_{2}(p), & \text { if } 1 / 2 \leq q \leq 1 .\end{cases}
$$

Using the parametric representation, (abuse of) notation, and method in Section $\mathrm{V}-\mathrm{A}$ it can verified that $\mathcal{P}_{f_{A} f_{B}}=$ $\{(p, q): p=0$ or $q=0$ or $p=1\}$ and $\rho^{*}(p, q)=\left(h_{2}(p)+h_{2}(q)-R^{*}(p, q)\right)$ belongs to $\mathcal{F}\left(\mathcal{P}_{X Y}\right)$, where $\mathcal{P}_{X Y}=[0,1]^{2}$ is the same marginal-perturbations-closed family used in Section $\mathrm{V}$-A Therefore, $R^{*}=R_{\text {sum, }, \infty}$.

Iterative algorithm: We will use this example to demonstrate how the iterative algorithm discussed in Section IV can be implemented on a computer.

- Initialization: Choose $\mathcal{P}_{X Y}=[0,1]^{2}$. Choose $\mathcal{A}=\{(1 / 2, q)\}_{q \in[0,1]}$, which leads to a cover for $\mathcal{P}_{X Y}$ made up of $X$-marginal perturbation sets $\{[0,1] \times\{q\}\}_{q \in[0,1]}$. Similarly, choose $\mathcal{B}=\{(p, 1 / 2)\}_{p \in[0,1]}$, which leads to a cover made up of $Y$-marginal perturbation sets $\{\{p\} \times[0,1]\}_{p \in[0,1]}$.

For computer-based numerical evaluation, discretize $\mathcal{P}_{X Y}$ into an $N \times N$ grid $\mathcal{P}_{X Y}^{*}:=\{(i /(N-1), j /(N-1))$ : $i, j=0, \ldots, N-1\}$. Correspondingly discretize the two covers are into the collection of the columns and the collection of the rows of $\mathcal{P}_{X Y}^{*}$. Compute $\rho_{0}^{A}(p, q)=\rho_{0}^{B}(p, q)=\rho_{0}(p, q)$ using equation (4) for all $(p, q) \in \mathcal{P}_{X Y}^{*}$ as follows:

$$
\rho_{0}(p, q)=\left\{\begin{array}{cc}
h_{2}(p)+h_{2}(q), & \text { if } p=0 \text { or } q=0 \text { or } p=1, \\
-\infty, & \text { otherwise. }
\end{array}\right.
$$

- Loop: For $\tau=1$ through $t$ do the following.

- For every $q \in\{j /(N-1): j=0, \ldots, N-1\}$, do the following. Let $\mathcal{H}_{q}\left(\rho_{\tau-1}^{B}\right):=\left\{\left(p, \rho_{\tau-1}^{B}(p, q)\right): p=\right.$ $\left.i /(N-1), i=0, \ldots, N-1, \rho_{\tau-1}^{B}(p, q) \neq-\infty\right\}$. Take the convex hull of $\mathcal{H}_{q}\left(\rho_{\tau-1}^{B}\right)$ and denote it by $\operatorname{ch}\left(\mathcal{H}_{q}\left(\rho_{\tau-1}^{B}\right)\right)$. For every $p \in\{i /(N-1): i=0, \ldots, N-1\}$,

$$
\rho_{\tau}^{A}(p, q):=\left\{\begin{array}{cc}
-\infty, & \text { if }\left\{\rho:(p, \rho) \in \operatorname{ch}\left(\mathcal{H}_{q}\left(\rho_{\tau-1}^{B}\right)\right)\right\}=\varnothing, \\
\max \left\{\rho:(p, \rho) \in \operatorname{ch}\left(\mathcal{H}_{q}\left(\rho_{\tau-1}^{B}\right)\right)\right\}, & \text { otherwise. }
\end{array}\right.
$$

By the above definition, $\left(p, \rho_{\tau}^{A}\right)$ is on the upper boundary of $\operatorname{ch}\left(\mathcal{H}_{q}\left(\rho_{\tau-1}^{B}\right)\right)$, taking the symbol $-\infty$ into consideration.

- For every $p \in\{i /(N-1): i=0, \ldots, N-1\}$, do the following. Let $\mathcal{H}_{p}\left(\rho_{\tau-1}^{A}\right):=\left\{\left(q, \rho_{\tau-1}^{A}(p, q)\right): q=j /(N-\right.$ $\left.1), j=0, \ldots, N-1, \rho_{\tau-1}^{A}(p, q) \neq-\infty\right\}$. Take the convex hull of $\mathcal{H}_{p}\left(\rho_{\tau-1}^{A}\right)$ and denote it by $\operatorname{ch}\left(\mathcal{H}_{p}\left(\rho_{\tau-1}^{A}\right)\right)$. For every $q \in\{j /(N-1): j=0, \ldots, N-1\}$,

$$
\rho_{\tau}^{B}(p, q):=\left\{\begin{array}{cc}
-\infty, & \text { if }\left\{\rho:(q, \rho) \in \operatorname{ch}\left(\mathcal{H}_{p}\left(\rho_{\tau-1}^{A}\right)\right)\right\}=\varnothing, \\
\max \left\{\rho:(q, \rho) \in \operatorname{ch}\left(\mathcal{H}_{p}\left(\rho_{\tau-1}^{A}\right)\right)\right\}, & \text { otherwise. }
\end{array}\right.
$$

- Optimality test: if $\forall(p, q) \in \mathcal{P}_{X Y}^{*}, \rho_{\tau}^{A}(p, q)=\rho_{\tau-1}^{B}(p, q)$ then set $\rho_{t}^{A}=\rho_{t}^{B}=\rho_{\tau}^{A}$ and exit the loop. if $\forall(p, q) \in \mathcal{P}_{X Y}^{*}, \rho_{\tau}^{B}(p, q)=\rho_{\tau-1}^{A}(p, q)$ then set $\rho_{t}^{A}=\rho_{t}^{B}=\rho_{\tau}^{B}$ and exit the loop.

- Output: $R_{\text {sum }, t}^{A}(p, q)=h_{2}(p)+h_{2}(q)-\rho_{t}^{A}(p, q)$ and $R_{\text {sum }, t}^{B}(p, q)=h_{2}(p)+h_{2}(q)-\rho_{t}^{B}(p, q)$ for all $(p, q) \in \mathcal{P}_{X Y}^{*}$.

Figure 2 shows mesh plots of the rate reduction function for different values of $t$. While the plots for $\rho_{0}$ and $\rho_{\infty}$ were generated using the closed-form expressions in (8) and (7), the remaining plots were generated numerically 

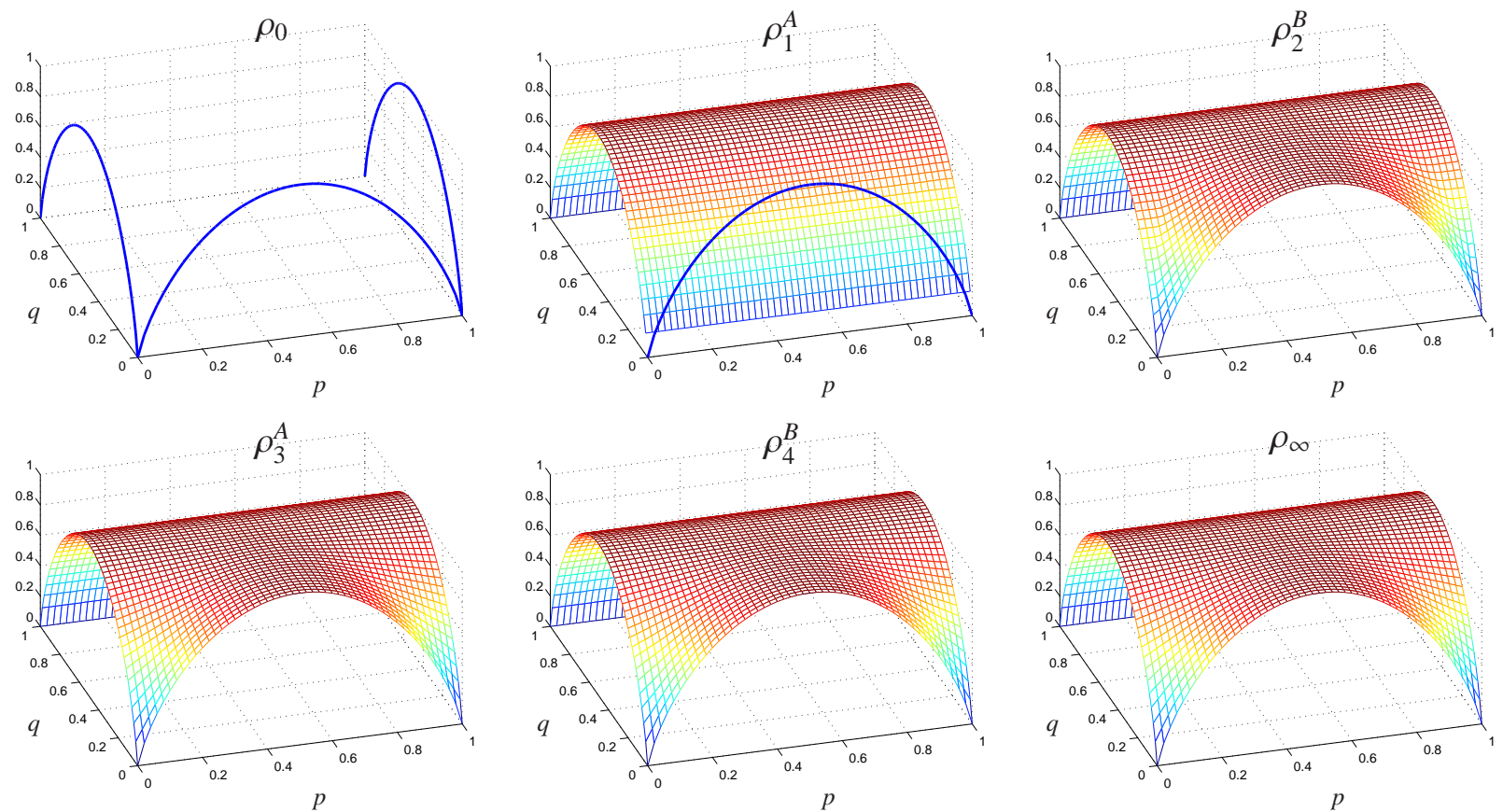

Fig. 2. Mesh plots of the rate reduction function for different number of messages $t$ for the example in Section $V-B$ Plots for $\rho_{0}$ and $\rho_{\infty}$ were generated using the closed-form expressions in (8) and (7). The remaining plots were generated numerically on a computer using the "alternating marginal concavification" algorithm described in Section $V-B$

on a computer by the iterative algorithm. Figure 2 enables one to explicitly visualize the "alternating marginal concavification" process: $\rho_{1}^{A}$ is obtained from $\rho_{0}$ by finding the "lowest" surface, not lower than $\rho_{0}$, which is concave along the $p$-axis for each value of $q ; \rho_{2}^{B}$ is obtained from $\rho_{1}^{A}$ by finding the "lowest" surface, not lower than $\rho_{1}^{A}$, which is concave along the $q$-axis for each value of $p$; and so on.

For $t \geq 2$, the points at which successive rate reduction functions differ from $\rho_{\infty}$ are only barely distinguishable (visually) in Figure 2. To better visualize the rate of convergence, in Figure 3 we plot the differences $\left(\rho_{\infty}-\rho_{t}\right)=$ $\left(R_{\text {sum }, t}-R_{\text {sum }, \infty}\right), t=1,2, \ldots$, as grayscale images where the brightness at any coordinate $(p, q)$ is proportional to $\log \left(\rho_{\infty}(p, q)-\rho_{t}(p, q)\right)$. Brighter (whiter) shades correspond to larger differences between $\rho_{\infty}$ and $\rho_{t}$ while a pure-black shade corresponds to a difference that is smaller than $10^{-4}$.

Reaching $R_{\text {sum }, \infty}$ with finite $t$ : For some joint source pmfs, the limit $R_{\text {sum }, \infty}$ can possibly be reached by $R_{\text {sum }, t}$ with a finite $t$. Specifically,

(i) For all $(p, q) \in \mathcal{P}_{f_{A} f_{B}}, R_{\text {sum }, 0}=0=R_{\text {sum }, \infty}$ and no message needs to be sent.

(ii) For all $(p, q) \in(0,1) \times[1 / 2,1], R_{\text {sum }, \infty}=h_{2}(p)$ and this sum-rate can be achieved with $t=1$ message from $A$ to $B$. Thus $R_{\text {sum }, 1}^{A}=R_{\text {sum }, \infty}$. However, note that $R_{\text {sum }, 1}^{B}=\infty$ and because $(p, q) \notin \mathcal{P}_{f_{A} f_{B}}, R_{\text {sum }, 0}=\infty$.

(iii) For $(p, q) \in\{1 / 2\} \times(0,1 / 2), R_{\text {sum }, \infty}=h_{2}(q)$. In [8, Sec. V.C] it was shown that this sum-rate can be achieved with $t=2$ messages, the first from $B$ to $A$ and the second from $A$ to $B$. Thus $R_{\text {sum }, 2}^{B}=R_{\text {sum }, \infty}$. We note that $R_{\text {sum }, 1}^{B}=\infty$ and in [1, Sec. IV.C] it was shown that $R_{\text {sum }, 1}^{A}=\log _{2} 2=1$.

Thus for the distributions $(p, q)$ discussed above, $R_{\text {sum }, \infty}$ can be reached with $t=0,1$ or 2 messages. However, Figure 3 also shows that for values of $(p, q)$ that are close to the line segments $p=q<1 / 2$ and $(1-p)=q<1 / 2$, even when $t=8, R_{\text {sum }, t}$ is not very close to $R_{\text {sum }, \infty}$. We conjecture that for the example considered in this section, 

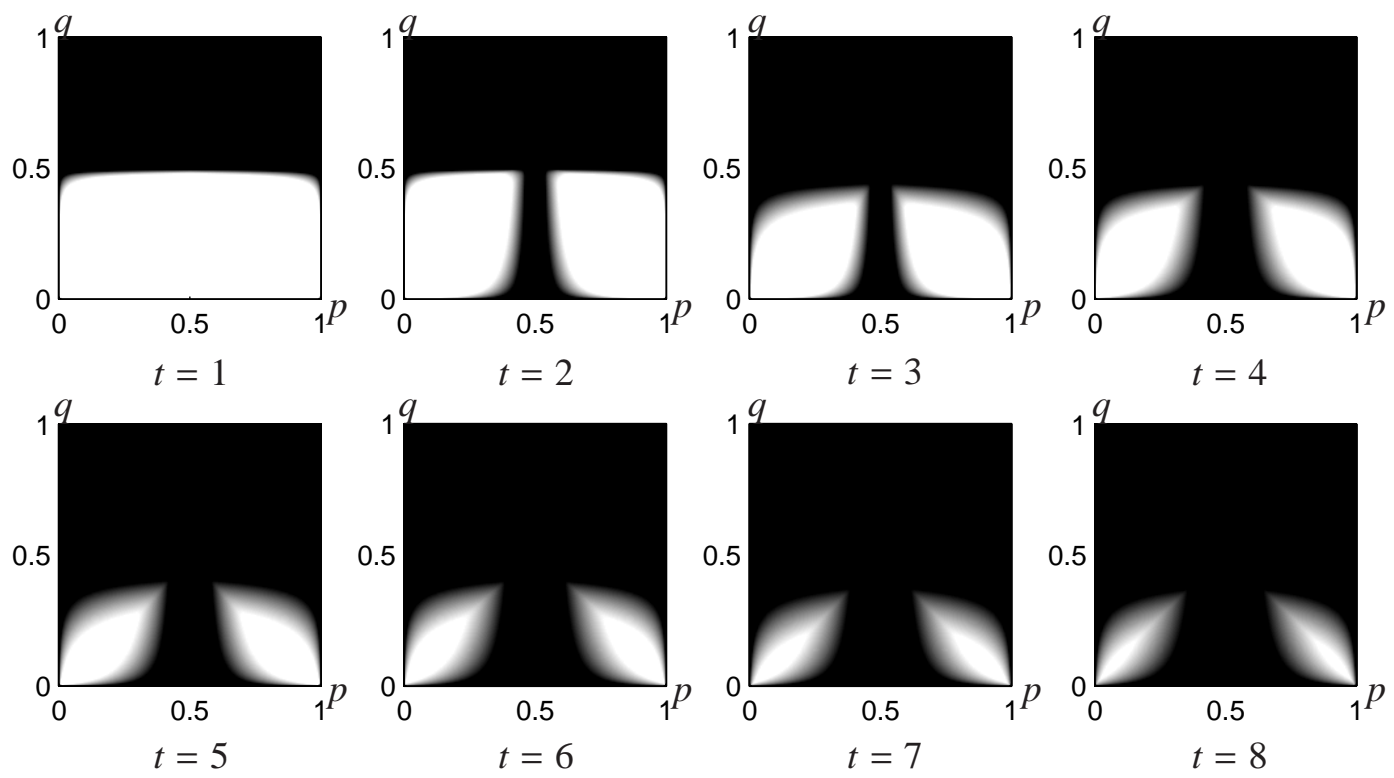

Fig. 3. For the example in Section $V-B$, the differences $\left(\rho_{\infty}-\rho_{t}\right)$, for $t=1,2, \ldots$, are plotted as grayscale images where the brightness at any coordinate $(p, q)$ is proportional to $\log \left(\rho_{\infty}(p, q)-\rho_{t}(p, q)\right)$. Brighter (whiter) shades correspond to larger differences between $\rho_{\infty}$ and $\rho_{t}$ while a pure-black shade corresponds to a difference that is smaller than $10^{-4}$.

there exist values of $(p, q)$ close to the line segments $p=q<1 / 2$ and $(1-p)=q<1 / 2$ for which $R_{\text {sum }, t}(p, q)$ is strictly smaller than $R_{\text {sum }, \infty}(p, q)$ for all finite values of $t$, i.e., an infinite number of messages are necessary to reach the infinite-message limit.

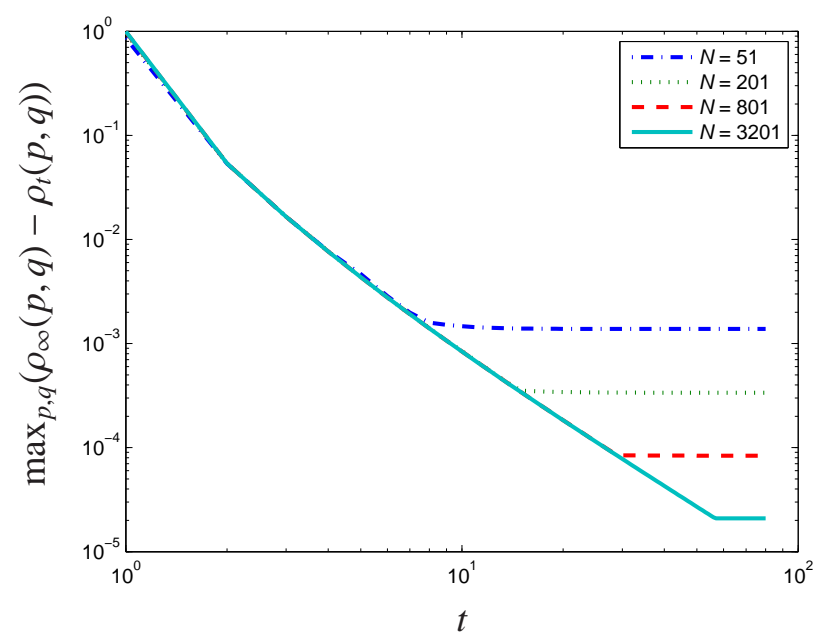

(a)

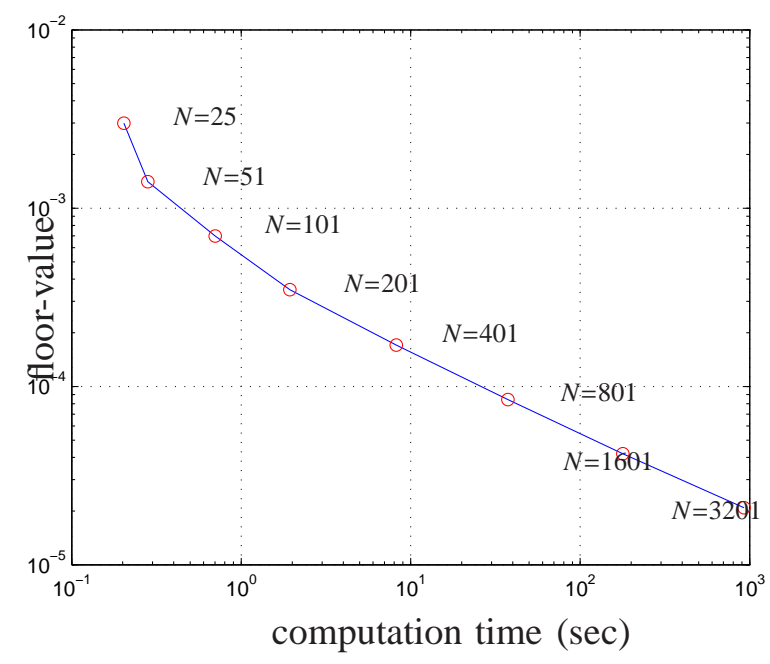

(b)

Fig. 4. (a) Plot showing how $\max _{p, q}\left(\rho_{\infty}(p, q)-\rho_{t}(p, q)\right)$ monotonically decreases as the number of messages $t$ increases and reaches a floor whose value depends on the number of grid points $N^{2}$ in the discretization of $\mathcal{P}_{X Y}$ (see Section $V$-B . (b) Plot showing the relationship between the floor-value (due to discretization) in plot (a) and the computation time needed to reach the floor-value. When $N$ is doubled, the floor-value is roughly halved and the computation time needed to reach the floor-value is roughly quadrupled.

Discretization step-size and floor-value: The time complexity of the algorithm depends on the way $\mathcal{P}_{X Y}$ is 
discretized into $\mathcal{P}_{X Y}^{*}$. In this particular example, the time complexity as a function of the discretization parameter $N$ and the number of iterations $t$ can be analyzed as follows. Since finding the convex hull of $N$ points in a plane takes $O(N \log N)$ operations [13], and in each iteration $N$ convex hulls need to be computed, the time complexity to complete $t$ iterations is $O\left(t N^{2} \log N\right)$.

Ideally, for all $(p, q) \in[0,1]^{2}$, as $t$ increases, the difference between $\rho_{\infty}(p, q)$ and $\rho_{t}(p, q)$ should keep decreasing until it becomes zero. However, due to the discretization of $\mathcal{P}_{X Y}$, the difference does not monotonically decrease to zero but saturates around a discretization-induced floor-value. Figure 4 (a) shows how $\max _{p, q}\left(\rho_{\infty}(p, q)-\rho_{t}(p, q)\right)$ decreases as $t$ increases and reaches a floor whose value depends on $N$. In this numerical experiment, as $t$ increases, $\max _{p, q}\left(\rho_{\infty}(p, q)-\rho_{t}(p, q)\right)$ decreases roughly as $1 / t^{2}$ before reaching a floor value. The finer is the discretization (larger the $N$ ), the lower is the floor-value. Figure 4(b) shows how the floor-value behaves in relation to the computation time 2 needed to reach it and the corresponding value of $N$. Roughly speaking, when $N$ is doubled, the floor-value is halved and the computation time needed to reach the floor-value is quadrupled. These are purely empirical observations of the convergence-behavior restricted to the example of this section. A theoretical analysis of the rate of convergence of the proposed iterative algorithm for general problems is not established here and is left for future work.

\section{WeIGHTED MiNIMUM SUM-RATE AND DIRECTED SUM-RATE REGION}

The discussion thus far has focused on the minimum sum-rate as the overall measure of efficiency, where the rates of messages from terminal $A$ to $B$ and from terminal $B$ to $A$ are added up with the same unit weight. This is appropriate for the scenario where the costs of communication in both directions are the same. In some applications, however, the communication costs in different directions can be different. For example, in the communication between a cellphone and a base station, the cellphone has a stringent battery constraint whereas the base station does not. Therefore each bit sent by the cellphone is more expensive. In this section, we show how results from previous sections can be extended to such an asymmetric communication scenario.

\section{A. Weighted minimum sum-rate}

Unequal communication costs from $A$ to $B$ and $B$ to $A$ can be addressed by introducing nonnegative weights $k_{A B}$ and $k_{B A}$ respectively. For initial terminal $A$ and nonnegative weights $\left(k_{A B}, k_{B A}\right)$, the weighted minimum sum-rate and the weighted rate reduction can be defined, respectively, as follows

$$
\begin{aligned}
R_{\text {sum }, t}^{A, k_{A B}, k_{B A}} & :=\min _{\mathbf{R} \in \mathcal{R}_{t}^{A}}\left(\sum_{i \text { odd }} k_{A B} R_{i}+\sum_{i \text { even }} k_{B A} R_{i}\right), \\
\rho_{t}^{A, k_{A B}, k_{B A}}: & :=k_{A B} H(X \mid Y)+k_{B A} H(Y \mid X)-R_{\text {sum }, t}^{A, k_{A B}, k_{B A}},
\end{aligned}
$$

where, $\mathcal{R}_{t}^{A}$ is the set of all admissible rate tuples $\mathbf{R}$. Comparing these definitions with those of $R_{\text {sum }, t}^{A}$ and $\rho_{t}^{A}$, it follows that the single-letter characterizations of $R_{\text {sum, }}^{A, k_{A B}, k_{B A}}$ and $\rho_{t}^{A, k_{A B}, k_{B A}}$ can be obtained from (1) and (3) by scaling the terms corresponding to the rates of messages from $A$ to $B$ and from $B$ to $A$ by the weights $k_{A B}$ and $k_{B A}$

\footnotetext{
${ }^{2}$ The computation time is obtained using Matlab R2009a, a Pentium 4 CPU with clock rate 2.80GHz and 2GB RAM.
} 
respectively:

$$
\begin{aligned}
& R_{\text {sum,t }}^{A, k_{A B}, k_{B A}}=\min _{p_{U^{t} \mid X Y} \in \mathcal{P}_{t}^{A}\left(p_{X Y}\right)}\left[k_{A B} I\left(X ; U^{t} \mid Y\right)+k_{B A} I\left(Y ; U^{t} \mid X\right)\right], \\
& \rho_{t}^{A, k_{A B}, k_{B A}}=\max _{p_{U^{t} \mid X Y} \in \mathcal{P}_{t}^{A}\left(p_{X Y}\right)}\left[k_{A B} H\left(X \mid Y, U^{t}\right)+k_{B A} H\left(Y \mid X, U^{t}\right)\right] .
\end{aligned}
$$

For initial terminal $B$, the operational definitions and single-letter characterizations of $R_{s u m, t}^{B, k_{A B}, k_{B A}}$ and $\rho_{t}^{B, k_{A B}, k_{B A}}$ are analogous.

As in the symmetric communication scenario, the infinite-message limits $R_{\text {sum, }}^{k_{A B}, k_{B A}}$ and $\rho_{\infty}^{k_{A B}, k_{B A}}$ are respectively defined as the limits of $R_{s u m, t}^{A, k_{A B}, k_{B A}}$ and $\rho_{t}^{A, k_{A B}, k_{B A}}$ as $t \rightarrow \infty$. The functional $\rho_{\infty}^{k_{A B}, k_{B A}}\left(p_{X Y}\right)$ can be characterized by the following extension of Theorem 1

Theorem 2: If $\mathcal{F}^{k_{A B}, k_{B A}}\left(\mathcal{P}_{X Y}\right)$ denotes a family of functionals that satisfies Definition 3 with $\rho_{0}$ replaced by $\rho_{0}^{k_{A B}, k_{B A}}$ then $\rho_{\infty}^{k_{A B}, k_{B A}}$ is the least element of the set $\mathcal{F}^{k_{A B}, k_{B A}}\left(\mathcal{P}_{X Y}\right)$ with majorization as the partial ordering relation.

The proof of Theorem 2 is parallel to that of Theorem 11 with the weights $k_{A B}$ and $k_{B A}$ applied, respectively, to all the terms corresponding to rates from $A$ to $B$ and from $B$ to $A$.

Similarly, with $\rho_{0}$ replaced by $\rho_{0}^{k_{A B}, k_{B A}}$ in the initialization step, the iterative algorithm described in Section IV can be suitably modified to compute the weighted minimum sum-rate.

\section{B. Directed sum-rate region}

A finer characterization of the tradeoff between the rates in two directions is the two dimensional directed sumrate region defined by $\mathcal{R}_{\text {sum }, t}^{A}:=\left\{\left(\sum_{i \text { odd }} R_{i}, \sum_{i \text { even }} R_{i}\right): \mathbf{R} \in \mathcal{R}_{t}^{A}\right\}$. The first component of $\mathcal{R}_{\text {sum }, t}^{A}$ is the directed sum-rate of all messages from $A$ to $B$. The second component is the directed sum-rate of all messages from $B$ to $A$. This characterization is coarser than the complete $t$-dimensional rate region $\mathcal{R}_{t}^{A}$ where each component corresponds to the rate of a single message, but finer than the minimum sum-rate $R_{\text {sum }, t}^{A}$.

The weighted sum-rate can be used to characterize the directed sum-rate region as follows.

Theorem 3: For all $t \in\{0\} \cup \mathbb{Z}^{+}, \mathcal{R}_{\text {sum }, t}^{A}=\left\{\left(R_{A B}, R_{B A}\right): \forall \theta \in[0, \pi / 2], R_{A B} \cos \theta+R_{B A} \sin \theta \geq R_{\text {sum }, t}^{A, \cos \theta \sin \theta}\right\}$.

Proof: When the function computation problem is infeasible, (i) $\mathcal{R}_{\text {sum }, t}^{A}$ is empty and (ii) $\forall \theta \in[0, \pi / 2]$, $R_{\text {sum }, t}^{A, \cos \theta \sin \theta}=\infty$, so that the set appearing in the right side of the equation in Theorem 3 is empty. Therefore Theorem 3 holds when the computation is infeasible.

When the function computation problem is feasible, $\mathcal{R}_{\text {sum }, t}^{A}$ is nonempty. Since $\mathcal{R}_{t}^{A}$ is convex and closed, a linear projection of this set to a lower dimensional space is also convex and closed. Since $\mathcal{R}_{\text {sum }, t}^{A}$ is a linear projection of $\mathcal{R}_{t}^{A}$ to $\mathbb{R}^{2}$, it is convex and closed. Since every closed convex subset of $\mathbb{R}^{n}$ is the intersection of the halfspaces that contain it [14, Proposition B.15], $\mathcal{R}_{\text {sum }, t}^{A}$ is the intersection of all the halfspaces of $\mathbb{R}^{2}$ that contain $\mathcal{R}_{\text {sum }, t}^{A}$.

Every halfspace in $\mathbb{R}^{2}$ can be written in the following form: $\left\{\left(R_{A B}, R_{B A}\right): R_{A B} \cos \theta+R_{B A} \sin \theta \geq c\right\}$, where $\theta \in[0,2 \pi)$ describes the orientation of the boundary of the halfspace and $c \in \mathbb{R}$ describes the displacement of the boundary from the origin. Among all the halfspaces with a common orientation parameter $\theta$ in the range $[0, \pi / 2]$, the one with the displacement parameter $c=R_{\text {sum }, t}^{A, \cos \theta, \sin \theta}$ is the tightest halfspace that contains $\mathcal{R}_{\text {sum, } t}^{A}$. This follows by first noting that $\cos \theta$ and $\sin \theta$ are both nonnegative for $\theta \in[0, \pi / 2]$ and then setting $k_{A B}=\cos \theta$ and $k_{B A}=\sin \theta$ in (9). There is no halfspace that contains $\mathcal{R}_{\text {sum }, t}^{A}$ with an orientation parameter $\theta$ in the range $(\pi / 2,2 \pi)$, 
because in this range, either $\cos \theta<0$ or $\sin \theta<0$ and for sufficiently large values of $R_{A B}$ or $R_{B A} 3$ the inequality $R_{A B} \cos \theta+R_{B A} \sin \theta \geq c$ will be violated for any value of $c$. Therefore the intersection of all the halfspaces that contain $\mathcal{R}_{\text {sum }, t}^{A}$ is the intersection of the halfspaces with orientation $\theta \in[0, \pi / 2]$ and displacement $c=R_{\text {sum, }}^{A, \cos \theta \sin \theta}$.

Since null messages are permitted, we have $\mathcal{R}_{\text {sum }, t}^{A} \subseteq \mathcal{R}_{\text {sum }, t+1}^{A}$ and $\mathcal{R}_{\text {sum }, t}^{A} \subseteq \mathcal{R}_{\text {sum }, t+1}^{B}$ (see [1, Proposition 1] for a detailed discussion). We can consider the directed sum-rate region in the limit $t$ going to infinity. Define $\mathcal{R}_{\text {sum, } \infty}$ as the closure of $\bigcup_{t=1}^{\infty} \mathcal{R}_{\text {sum }, t}^{A}$. We can characterize $\mathcal{R}_{\text {sum }, \infty}$ using $R_{\text {sum }, \infty}^{\cos \theta} \sin \theta$ as follows.

Theorem 4: $\mathcal{R}_{\text {sum }, \infty}=\left\{\left(R_{A B}, R_{B A}\right): \forall \theta \in[0, \pi / 2], R_{A B} \cos \theta+R_{B A} \sin \theta \geq R_{\text {sum }, \infty}^{\cos \theta \sin \theta}\right\}$.

Proof: Since $\left\{\mathcal{R}_{\text {sum }, t}^{A}\right\}_{t=1}^{\infty}$ is a sequence of expanding convex sets, it can be verified that the union of them is also convex. Since $\mathcal{R}_{\text {sum }, \infty}$ is defined as the closure of $\left\{\mathcal{R}_{\text {sum }, t}^{A}\right\}_{t=1}^{\infty}$, it is both closed and convex. The rest of the proof can be completed using the arguments from Theorem 3.

Combining Theorems 2 and 4 we have a characterization of $\mathcal{R}_{\text {sum, } \infty}$ as a functional of $p_{X Y}$. We can also numerically evaluate $\rho_{\infty}^{\cos \theta, \sin \theta}$ using an iterative algorithm that would be analogous to that in Section IV This would, in turn, provide a means to numerically evaluate $\mathcal{R}_{\text {sum }, \infty}$.

\section{EXTENSION TO INTERACTIVE RATE-DISTORTION PROBLEM}

\section{A. Problem formulation}

In [2] we studied the interactive coding problem with per-sample distortion criteria. Let $d_{A}: \mathcal{X} \times \mathcal{Y} \times \mathcal{Z}_{A} \rightarrow \mathcal{D}$ and $d_{B}: \mathcal{X} \times \mathcal{Y} \times \mathcal{Z}_{B} \rightarrow \mathcal{D}$ be bounded single-letter distortion functions, where $\mathcal{D}:=\left[0, d_{\max }\right]$. The fidelity of function computation can be measured by the per-sample average distortion

$$
d_{A}^{(n)}\left(\mathbf{x}, \mathbf{y}, \hat{\mathbf{z}}_{A}\right):=\frac{1}{n} \sum_{i=1}^{n} d_{A}\left(x(i), y(i), \hat{z}_{A}(i)\right), \quad d_{B}^{(n)}\left(\mathbf{x}, \mathbf{y}, \hat{\mathbf{z}}_{B}\right):=\frac{1}{n} \sum_{i=1}^{n} d_{B}\left(x(i), y(i), \hat{z}_{B}(i)\right) .
$$

Of interest here are the expected per-sample distortions $E\left[d_{A}^{(n)}\left(\mathbf{X}, \mathbf{Y}, \widehat{\mathbf{Z}}_{A}\right)\right]$ and $E\left[d_{B}^{(n)}\left(\mathbf{X}, \mathbf{Y}, \widehat{\mathbf{Z}}_{B}\right)\right]$. We note that although the desired functions $f_{A}$ and $f_{B}$ do not explicitly appear in these fidelity criteria, they are subsumed by $d_{A}$ and $d_{B}$ because they accommodate general relationships between the sources and the outputs of the decoding functions. The performance of $t$-message interactive coding for function computation is measured as follows.

Definition 4: A rate-distortion tuple $(\mathbf{R}, \mathbf{D})=\left(R_{1}, \ldots, R_{t}, D_{A}, D_{B}\right)$ is admissible for $t$-message interactive function computation with initial terminal $A$ if, $\forall \epsilon>0, \exists N(\epsilon, t)$ such that $\forall n>N(\epsilon, t)$, there exists an interactive distributed source code with initial terminal $A$ and parameters $\left(t, n,\left|\mathcal{M}_{1}\right|, \ldots,\left|\mathcal{M}_{t}\right|\right)$ satisfying

$$
\begin{aligned}
& \frac{1}{n} \log _{2}\left|\mathcal{M}_{j}\right| \leq R_{j}+\epsilon, j=1, \ldots, t, \\
& E\left[d_{A}^{(n)}\left(\mathbf{X}, \mathbf{Y}, \widehat{\mathbf{Z}}_{A}\right)\right] \leq D_{A}+\epsilon, E\left[d_{B}^{(n)}\left(\mathbf{X}, \mathbf{Y}, \widehat{\mathbf{Z}}_{B}\right)\right] \leq D_{B}+\epsilon .
\end{aligned}
$$

The set of all admissible rate-distortion tuples, denoted by $\mathcal{R} \mathcal{D}_{t}^{A}$, is called the operational rate-distortion region for $t$-message interactive function computation with initial terminal $A$. The rate-distortion region is closed and convex due to the way it has been defined. The sum-rate-distortion function $R_{\text {sum }, t}^{A}(\mathbf{D})$ is given by $\min \left(\sum_{j=1}^{t} R_{j}\right)$ where the minimization is over all $\mathbf{R}$ such that $(\mathbf{R}, \mathbf{D}) \in \mathcal{R} \mathcal{D}_{t}^{A}$. For initial terminal $B$, the rate-distortion region and the minimum sum-rate-distortion function are denoted by $\mathcal{R} \mathcal{D}_{t}^{B}$ and $R_{\text {sum }, t}^{B}(\mathbf{D})$ respectively. For any fixed $\mathbf{D}$, We define $R_{\text {sum }, \infty}(\mathbf{D}):=\lim _{t \rightarrow \infty} R_{\text {sum }, t}^{A}(\mathbf{D})=\lim _{t \rightarrow \infty} R_{\text {sum }, t}^{B}(\mathbf{D})$. Results of this section for the sum-rate-distortion

${ }^{3}$ Since $\mathcal{R}_{\text {sum }, t}^{A}$ is nonempty, there exists $\left(R_{A B}^{*}, R_{B A}^{*}\right) \in \mathcal{R}_{\text {sum }, t}^{A}$. According to the definition of a rate region, $\forall R_{A B} \geq R_{A B}^{*}$ and $\forall R_{B A} \geq R_{B A}^{*}$, we have $\left(R_{A B}, R_{B A}\right) \in \mathcal{R}_{\text {sum }, t}^{A}$. 
function can be generalized to corresponding results for the weighted sum-rate-distortion function and the directed sum-rate-distortion region in the same manner that results for the minimum sum-rate function were generalized to corresponding results for the weighted minimum sum-rate function and the directed sum-rate region in Sections VI-A and VI-B

The admissibility of a rate-distortion tuple can also be defined in terms of the probability of excess distortion by replacing the expected distortion conditions in Definition 4 by the conditions $\mathbb{P}\left(d_{A}^{(n)}\left(\mathbf{X}, \mathbf{Y}, \widehat{\mathbf{Z}}_{A}\right)>D_{A}\right) \leq \epsilon$ and $\mathbb{P}\left(d_{B}^{(n)}\left(\mathbf{X}, \mathbf{Y}, \widehat{\mathbf{Z}}_{B}\right)>D_{B}\right) \leq \epsilon$. Although these conditions appear to be more stringen 4 , it can be shown 5 that they lead to the same operational rate-distortion region. For simplicity, we focus on the expected distortion conditions as in Definition 4

\section{B. Characterization of $R_{\text {sum }, t}^{A}\left(p_{X Y}, \mathbf{D}\right)$ and $\rho_{t}^{A}\left(p_{X Y}, \mathbf{D}\right)$ for finite $t[2]$}

The single-letter characterization of $R_{\text {sum }, t}^{A}\left(p_{X Y}, \mathbf{D}\right)$ is given by

$$
R_{\text {sum }, t}^{A}\left(p_{X Y}, \mathbf{D}\right)=\min _{\left(p_{U^{t} \mid X Y}, \hat{g}_{A}, \hat{g}_{B}\right) \in \mathcal{P}_{t}^{A}\left(p_{X Y}, \mathbf{D}\right)}\left[I\left(X ; U^{t} \mid Y\right)+I\left(Y ; U^{t} \mid X\right)\right],
$$

where $\mathcal{P}_{t}^{A}\left(p_{X Y}, \mathbf{D}\right)$ is the set of all tuples $\left(p_{U^{t} \mid X Y}, \hat{g}_{A}, \hat{g}_{B}\right)$ such that (i) $\hat{g}_{A}$ and $\hat{g}_{B}$ are deterministic functions and $\left.E\left[d_{A}\left(X, Y, \hat{g}_{A}\left(U^{t}, X\right)\right)\right] \leq D_{A}, E\left[d_{B}\left(X, Y, \hat{g}_{B}\left(U^{t}, Y\right)\right)\right] \leq D_{B}\right\}$, (ii) for $i=1, \ldots, t$, if $i$ is odd, $U_{i}-\left(X, U^{i-1}\right)-Y$ forms a Markov chain, otherwise $U_{i}-\left(Y, U^{i-1}\right)-X$ forms a Markov chain, and (iii) $\left|\mathcal{U}_{1}\right|, \ldots,\left|\mathcal{U}_{t}\right|$ are finite alphabets whose cardinality satisfy (2). Compared to (1), the expected distortion constraints have replaced the conditional entropy constraints in (10). The rate reduction functional is defined as follows.

$$
\rho_{t}^{A}\left(p_{X Y}, \mathbf{D}\right):=H(X \mid Y)+H(Y \mid X)-R_{\text {sum }, t}^{A}(\mathbf{D})=\max _{\left(p_{U^{t} \mid X Y}, \hat{g}_{A}, \hat{g}_{B}\right) \in \mathcal{P}_{t}^{A}\left(p_{X Y}, \mathbf{D}\right)}\left[H\left(X \mid Y, U^{t}\right)+H\left(Y \mid X, U^{t}\right)\right] .
$$

For $t=0$, let $\mathcal{P}_{f_{A} f_{B} D}:=\left\{\left(p_{X Y}, \mathbf{D}\right): \exists \hat{g}_{A}, \hat{g}_{B}\right.$, s.t. $\left.E\left[d_{A}\left(X, Y, \hat{g}_{A}(X)\right)\right] \leq D_{A}, E\left[d_{B}\left(X, Y, \hat{g}_{B}(Y)\right)\right] \leq D_{B}\right\}$. Then we have

$$
\begin{gathered}
R_{\text {sum }, 0}\left(p_{X Y}, \mathbf{D}\right)=\left\{\begin{array}{cc}
0, & \text { if }\left(p_{X Y}, \mathbf{D}\right) \in \mathcal{P}_{f_{A} f_{B} D}, \\
+\infty, & \text { otherwise. }
\end{array}\right. \\
\rho_{0}\left(p_{X Y}, \mathbf{D}\right)=\left\{\begin{array}{cc}
H(X \mid Y)+H(Y \mid X), & \text { if }\left(p_{X Y}, \mathbf{D}\right) \in \mathcal{P}_{f_{A} f_{B} D}, \\
-\infty, & \text { otherwise. }
\end{array}\right.
\end{gathered}
$$

\section{Characterization of $R_{\text {sum }, \infty}\left(p_{X Y}, \mathbf{D}\right)$}

We can use the same technique as in Section $\amalg$ to characterize the functional $\rho_{\infty}\left(p_{X Y}, \mathbf{D}\right)$.

Definition 5: (Marginal-perturbations-distortion-concave, $\rho_{0}$-majorizing family of functionals $\mathcal{F}_{D}\left(\mathcal{P}_{X Y}\right)$ ) Let $\mathcal{P}_{X Y}$ be any marginal-perturbations-closed family of joint pmfs on $\Delta(\mathcal{X} \times \mathcal{Y})$. The set of marginal-perturbations-distortionconcave, $\rho_{0}$-majorizing family of functionals $\mathcal{F}_{D}\left(\mathcal{P}_{X Y}\right)$ is the set of all the functionals $\rho: \mathcal{P}_{X Y} \times \mathcal{D}^{2} \rightarrow \mathbb{R}$ satisfying the following conditions:

1) $\rho_{0}$-majorization: $\forall p_{X Y} \in \mathcal{P}_{X Y}$ and $\forall \mathbf{D} \in \mathcal{D}^{2}, \rho\left(p_{X Y}, \mathbf{D}\right) \geq \rho_{0}\left(p_{X Y}, \mathbf{D}\right)$.

2) Concavity with respect to $X$-marginal perturbations and distortion vector: $\forall p_{X Y} \in \mathcal{P}_{X Y}, \rho$ is concave on $\mathcal{P}_{Y \mid X}\left(p_{X Y}\right) \times \mathcal{D}^{2}$.

${ }^{4}$ Any tuple which is admissible according to the probability of excess distortion criteria is also admissible according to the expected distortion criteria.

${ }^{5}$ Using strong-typicality arguments in the proof of the achievability part of the single-letter characterization of the rate-distortion region. 
3) Concavity with respect to $Y$-marginal perturbations and distortion vector: $\forall p_{X Y} \in \mathcal{P}_{X Y}, \rho$ is concave on $\mathcal{P}_{X \mid Y}\left(p_{X Y}\right) \times \mathcal{D}^{2}$.

The following characterization of $\rho_{\infty}\left(p_{X Y}, \mathbf{D}\right)$ is the generalization of Theorem 1 to the rate-distortion problem.

Theorem 5: (i) $\rho_{\infty}\left(p_{X Y}, \mathbf{D}\right) \in \mathcal{F}_{D}\left(\mathcal{P}_{X Y}\right)$. (ii) For all $\rho \in \mathcal{F}_{D}\left(\mathcal{P}_{X Y}\right)$ and $\forall\left(p_{X Y}, \mathbf{D}\right) \in \mathcal{P}_{X Y} \times \mathcal{D}^{2}$, we have $\rho_{\infty}\left(p_{X Y}, \mathbf{D}\right) \leq \rho\left(p_{X Y}, \mathbf{D}\right)$.

The proof of Theorem 5 is parallel to that of Theorem 1

Proof: First we establish the relation between $\rho_{t}^{A}$ and $\rho_{t-1}^{B}$ as follows.

Lemma 2: (i) For all $t \in \mathbb{Z}^{+}$and all $\left(p_{X Y}, \mathbf{D}\right) \in \mathcal{P}_{X Y} \times \mathcal{D}^{2}$,

$$
\rho_{t}^{A}\left(p_{X Y}, \mathbf{D}\right)=\max _{p_{U_{1} \mid X}}\left\{\max _{\substack{\forall u_{1} \in \mathcal{U}_{1}, \mathbf{D}_{u_{1}} \in \mathcal{D}^{2}: \\ E\left[\mathbf{D}_{U_{1}}\right]=\mathbf{D}}}\left\{\sum_{u_{1} \in \operatorname{supp}\left(p_{U_{1}}\right)} p_{U_{1}}\left(u_{1}\right) \rho_{t-1}^{B}\left(p_{X Y \mid U_{1}}\left(\cdot, \cdot \mid u_{1}\right), \mathbf{D}_{u_{1}}\right)\right\}\right\} .
$$

(ii) For all $t \in \mathbb{Z}^{+}$and all $\left(q_{X Y}, \mathbf{D}\right) \in \mathcal{P}_{X Y} \times \mathcal{D}^{2}, \rho_{t}^{A}$ is concave on $\mathcal{P}_{Y \mid X}\left(q_{X Y}\right) \times \mathcal{D}^{2}$.

(iii) For all $t \in \mathbb{Z}^{+}$and all $\left(q_{X Y}, \mathbf{D}\right) \in \mathcal{P}_{X Y} \times \mathcal{D}^{2}$, if $\rho: \mathcal{P}_{X Y} \times \mathcal{D}^{2} \rightarrow \mathbb{R}$ is concave on $\mathcal{P}_{Y \mid X}\left(q_{X Y}\right) \times \mathcal{D}^{2}$ and if for all $\left(p_{X Y}, \mathbf{D}\right) \in \mathcal{P}_{Y \mid X}\left(q_{X Y}\right) \times \mathcal{D}^{2}, \rho_{t-1}^{B}\left(p_{X Y}, \mathbf{D}\right) \leq \rho\left(p_{X Y}, \mathbf{D}\right)$, then for all $\left(p_{X Y}, \mathbf{D}\right) \in \mathcal{P}_{Y \mid X}\left(q_{X Y}\right) \times \mathcal{D}^{2}, \rho_{t}^{A}\left(p_{X Y}, \mathbf{D}\right) \leq$ $\rho\left(p_{X Y}, \mathbf{D}\right)$.

(iv) The results of parts (i) - (iii) above also hold if $A$ is swapped with $B$ and simultaneously, $\mathcal{P}_{Y \mid X}$ and $p_{U_{1} \mid X}$ are replaced by $\mathcal{P}_{X \mid Y}$ and $p_{U_{1} \mid Y}$ respectively.

Proof: See Appendix D.

Equipped with Lemma 2, the rest of the proof of Theorem 5 is parallel to that of Theorem 1 except that all the rate reduction functionals depend on $\left(p_{X Y}, \mathbf{D}\right)$ instead of only $p_{X Y}$.

The intuition underlying the proof of Theorem 5 is essentially conveyed by the first paragraph of the proof of Theorem 1 The main difference is that for each realization $U_{1}=u_{1}$, the distortion vector $\mathbf{D}_{u_{1}}$ in the $(t-1)$-message subproblem could be different from the original distortion vector $\mathbf{D}$. The only constraint that $\left\{\mathbf{D}_{u_{1}}\right\}_{u_{1} \in \mathcal{U}_{1}}$ needs to satisfy is that $\sum_{u_{1}} \mathbf{D}_{u_{1}} p_{U_{1}}\left(u_{1}\right)=\mathbf{D}$ holds. Therefore, we need to consider joint convex combinations of the distortion vector and the marginal source distributions.

The counterparts of Corollaries 1 to 3 in the rate-distortion problem also hold. The proofs are parallel to those of Corollaries 1 to 3

Corollary 4: (Constructing $\rho_{t}^{A}$ and $\rho_{t}^{B}$ from $\rho_{t-1}^{B}$ and $\rho_{t-1}^{A}$ respectively) For all $t \in \mathbb{Z}^{+}$and all $p_{X Y} \in \mathcal{P}_{X Y}$, we have (i) $\operatorname{ch}\left(\operatorname{hypo}_{\left(\mathcal{P}_{Y \mid X}\left(p_{X Y}\right) \times \mathcal{D}^{2}\right)} \rho_{t-1}^{B}\right)=\operatorname{hypo}_{\left(\mathcal{P}_{Y \mid X}\left(p_{X Y}\right) \times \mathcal{D}^{2}\right)} \rho_{t}^{A}$ and (ii) $\operatorname{ch}\left(\operatorname{hypo}_{\left(\mathcal{P}_{X \mid Y}\left(p_{X Y}\right) \times \mathcal{D}^{2}\right)} \rho_{t-1}^{A}\right)=\operatorname{hypo}_{\left(\mathcal{P}_{X \mid Y}\left(p_{X Y}\right) \times \mathcal{D}^{2}\right)} \rho_{t}^{B}$.

Corollary 5: (Concavity and optimality of $\rho_{t}^{A}$ and $\rho_{t}^{B}$ ) For all $t \in \mathbb{Z}^{+}$, the following conditions are equivalent:

(i) $\forall p_{X Y} \in \mathcal{P}_{X Y}, \forall \mathbf{D} \in \mathcal{D}^{2}, \rho_{t}^{A}\left(p_{X Y}, \mathbf{D}\right)=\rho_{\infty}\left(p_{X Y}, \mathbf{D}\right)$,

(ii) $\forall p_{X Y} \in \mathcal{P}_{X Y}, \forall \mathbf{D} \in \mathcal{D}^{2}, \rho_{t}^{A}\left(p_{X Y}, \mathbf{D}\right)=\rho_{t+1}^{B}\left(p_{X Y}, \mathbf{D}\right)$,

(iii) $\forall p_{X Y} \in \mathcal{P}_{X Y}, \rho_{t}^{A}$ is concave on $\mathcal{P}_{X \mid Y}\left(p_{X Y}\right) \times \mathcal{D}^{2}$.

For all $t \in \mathbb{Z}^{+}$, the following three conditions are also equivalent:

(iv) $\forall p_{X Y} \in \mathcal{P}_{X Y}, \forall \mathbf{D} \in \mathcal{D}^{2}, \rho_{t}^{B}\left(p_{X Y}, \mathbf{D}\right)=\rho_{\infty}\left(p_{X Y}, \mathbf{D}\right)$,

(v) $\forall p_{X Y} \in \mathcal{P}_{X Y}, \forall \mathbf{D} \in \mathcal{D}^{2}, \rho_{t}^{B}\left(p_{X Y}, \mathbf{D}\right)=\rho_{t+1}^{A}\left(p_{X Y}, \mathbf{D}\right)$,

(vi) $\forall p_{X Y} \in \mathcal{P}_{X Y}, \rho_{t}^{B}$ is concave on $\mathcal{P}_{Y \mid X}\left(p_{X Y}\right) \times \mathcal{D}^{2}$. 
Corollary 6: (Optimality test for an achievable sum-rate distortion function) Let $R^{*}$ be a sum-rate distortion function which is achievable using an arbitrary number of messages. If $\rho^{*}:=\left(H(X \mid Y)+H(Y \mid X)-R^{*}\right) \in \mathcal{F}_{D}\left(\mathcal{P}_{X Y}\right)$, then $R^{*}=R_{\text {sum }, \infty}$.

\section{Iterative algorithm for computing $R_{\text {sum }, t}^{A}\left(p_{X Y}, \mathbf{D}\right)$ and $R_{\text {sum }, \infty}\left(p_{X Y}, \mathbf{D}\right)$}

Corollary 4 suggests the following algorithm which is similar to the one presented in Section IV

Algorithm to evaluate $R_{\text {sum }, t}^{A}\left(p_{X Y}, \mathbf{D}\right)$ and $R_{\text {sum }, t}^{B}\left(p_{X Y}, \mathbf{D}\right)$

- Initialization: Choose a marginal-perturbations-closed family $\mathcal{P}_{X Y}$ containing all joint source pmfs of interest. Define $\rho_{0}^{A}\left(p_{X Y}, \mathbf{D}\right)=\rho_{0}^{B}\left(p_{X Y}, \mathbf{D}\right)=\rho_{0}\left(p_{X Y}, \mathbf{D}\right)$ by equation (11) in the domain $\mathcal{P}_{X Y} \times \mathcal{D}^{2}$. Choose a cover for $\mathcal{P}_{X Y}$ made up of $X$-marginal perturbation sets, denoted by $\left\{\mathcal{P}_{Y \mid X}\left(p_{X Y}\right)\right\}_{p_{X Y} \in \mathcal{A}}$, where $\mathcal{A} \subseteq \mathcal{P}_{X Y}$. Also choose a cover for $\mathcal{P}_{X Y}$ made up of $Y$-marginal perturbation sets, denoted by $\left\{\mathcal{P}_{X \mid Y}\left(p_{X Y}\right)\right\}_{p_{X Y} \in \mathcal{B}}$, where $\mathcal{B} \subseteq \mathcal{P}_{X Y}$.

- Loop: For $\tau=1$ through $t$ do the following.

For every $p_{X Y} \in \mathcal{A}$, do the following in the set $\mathcal{P}_{Y \mid X}\left(p_{X Y}\right) \times \mathcal{D}^{2}$.

- Construct hypo $\mathcal{P}_{Y \mid X}\left(p_{X Y}\right) \times \mathcal{D}^{2} \rho_{\tau-1}^{B}$.

- Let $\rho_{\tau}^{A}$ be the upper boundary of $\operatorname{ch}\left(\operatorname{hypo}_{\mathcal{P}_{Y \mid X}\left(p_{X Y}\right) \times \mathcal{D}^{2}} \rho_{\tau-1}^{B}\right)$.

For every $p_{X Y} \in \mathcal{B}$, do the following in the set $\mathcal{P}_{X \mid Y}\left(p_{X Y}\right) \times \mathcal{D}^{2}$.

- Construct hypo $\mathcal{P}_{X \mid Y}\left(p_{X Y}\right) \times \mathcal{D}^{2} \rho_{\tau-1}^{A}$.

- Let $\rho_{\tau}^{B}$ be the upper boundary of $\operatorname{ch}\left(\operatorname{hypo}_{\mathcal{P}_{X \mid Y}\left(p_{X Y}\right) \times \mathcal{D}^{2}} \rho_{\tau-1}^{A}\right)$.

Optimality test:

if $\forall\left(p_{X Y}, \mathbf{D}\right) \in \mathcal{P}_{X Y} \times \mathcal{D}^{2}, \rho_{\tau}^{A}\left(p_{X Y}, \mathbf{D}\right)=\rho_{\tau-1}^{B}\left(p_{X Y}, \mathbf{D}\right)$, then set $\rho_{t}^{A}=\rho_{t}^{B}=\rho_{\tau}^{A}$ and exit the loop.

if $\forall\left(p_{X Y}, \mathbf{D}\right) \in \mathcal{P}_{X Y} \times \mathcal{D}^{2}, \rho_{\tau}^{B}\left(p_{X Y}, \mathbf{D}\right)=\rho_{\tau-1}^{A}\left(p_{X Y}, \mathbf{D}\right)$, then set $\rho_{t}^{A}=\rho_{t}^{B}=\rho_{\tau}^{A}$ and exit the loop.

- Output: $R_{\text {sum }, t}^{A}\left(p_{X Y}, \mathbf{D}\right)=H(X \mid Y)+H(Y \mid X)-\rho_{t}^{A}\left(p_{X Y}, \mathbf{D}\right)$, and $R_{\text {sum }, t}^{B}\left(p_{X Y}, \mathbf{D}\right)=H(X \mid Y)+H(Y \mid X)-\rho_{t}^{B}\left(p_{X Y}, \mathbf{D}\right)$.

In a computer implementation, we will need to discretize the set $\mathcal{P}_{X Y} \times \mathcal{D}^{2} . R_{\text {sum, },}\left(p_{X Y}, \mathbf{D}\right)$ can, in principle, be evaluated to any precision by running this algorithm to a large enough value of $t$, until the change between $\rho_{t-1}^{A}\left(p_{X Y}, \mathbf{D}\right)$ and $\rho_{t}^{A}\left(p_{X Y}, \mathbf{D}\right)$ is below a certain threshold. In the special case $t=1$, and $d_{A} \equiv 0$, the interactive problem reduces to the Wyner-Ziv problem (with a general coupled distortion metric). If we further assume that $|y|=1$, the Wyner-Ziv problem reduces to the single-terminal rate-distortion problem. Therefore, the algorithm described above can be used to evaluate the single-terminal and Wyner-Ziv rate-distortion functions as special cases.

\section{THE BENEFIT OF INTERACTION FOR LOSSY SOURCE REPRODUCTION}

\section{A. Introducing the unresolved question}

The interactive rate-distortion problem defined in Section VII-A reduces to a lossy source reproduction problem when $d_{A}\left(x, y, z_{A}\right)$ depends only on $\left(y, z_{A}\right)$ and $d_{B}\left(x, y, z_{B}\right)$ depends only on $\left(x, z_{B}\right)$. In this special case, the single-letter characterization of the sum-rate-distortion function was given in [3] for all finite number of messages. Yet, whether more messages can strictly improve the sum-rate-distortion function was left unresolved. If the goal is to reproduce both sources losslessly at each terminal (zero distortion) then there is no advantage in using multiple messages; two messages are sufficient and the minimum sum-rate cannot be reduced by using more than two messages 6 If,

${ }^{6}$ If only one of the sources is required to be losslessly reproduced at the other terminal then one message is sufficient and the minimum sum-rate cannot be improved by using more than one message. 
however, the goal is changed to losslessly compute functions of sources at each terminal, then multiple messages can decrease the minimum sum-rate by an arbitrarily large factor [1], [8]. Therefore, the key unresolved question pertains to lossy source reproduction: can multiple messages strictly decrease the minimum sum-rate for a given (nonzero) distortion? This question was unresolved even when only one source needs to be reproduced with nonzero distortion.

In this section, we construct the first example which shows that two messages can strictly improve the onemessage (Wyner-Ziv) rate-distortion function. The example also shows that the ratio of the one-message rate to the two-message sum-rate can be arbitrarily large and simultaneously the ratio of the backward rate to the forward rate in the two-message sum-rate can be arbitrarily small. The rate reduction functional and its properties, specifically Corollary 5, play an important role in the construction of this example. In Section VIII-D we provide another example where in addition to the above properties, the one-message rate-distortion function can be arbitrarily large and the two-message sum-rate can be arbitrarily small.

In Section VIII-B and Section VIII-C we consider a lossy source reproduction problem where only terminal $B$ needs to reproduce $\mathbf{X}$ within distortion level $D=D_{B}$, and terminal $A$ is not required to reproduce anything $\left(d_{A} \equiv 0\right)$. In Theorem 6 , we will use Corollary 5 to show that there exist $p_{X Y}, d$, and $D$ for which $R_{\text {sum, }}^{A}\left(p_{X Y}, D\right)>$ $R_{\text {sum }, 2}^{B}\left(p_{X Y}, D\right)$. We will do this by (i) choosing $p_{X \mid Y}$ so that $X$ and $Y$ are symmetrically correlated binary random variables with $\mathbb{P}(Y \neq X)=p$, (ii) taking $d(x, \hat{x})$ to be the binary erasure distortion function, (iii) selecting a value for $D$, and (iv) showing that $\rho_{1}^{A}\left(p_{X \mid Y} p_{Y}, D\right)$ is not concave with respect to $p_{Y}$, which implies that condition (iii) of Corollary 5 does not hold for $t=1$. Therefore condition (ii) does not hold: $\rho_{1}^{A}\left(p_{X Y}, D\right) \neq \rho_{2}^{B}\left(p_{X Y}, D\right)$, which would imply that $R_{\text {sum }, 1}^{A}\left(p_{X Y}, D\right)>R_{\text {sum }, 2}^{B}\left(p_{X Y}, D\right)$. In Theorem 7 we will show that for certain values of parameters $p$ and $D$, the two-message sum-rate can be split in such a way that the ratio $R_{1} / R_{2}$ is arbitrarily small and simultaneously the ratio $R_{\text {sum }, 1}^{A} /\left(R_{1}+R_{2}\right)$ is arbitrarily large. This will be proved by explicitly constructing auxiliary variables $V_{1}, V_{2}$ 7 and decoding function $\hat{g}_{B}$. While the explicit construction of $V_{1}, V_{2}$ and $\hat{g}_{B}$ in the proof of Theorem 7 may make the implicit proof of Theorem 6 seem redundant, it is unclear how the explicit construction can be generalized to other families of source distributions and distortion functions. The approach followed in the proof of Theorem 6 on the other hand, provides an efficient method to test whether the best two-message scheme can strictly outperform the best one-message scheme for more general distributed source coding and function computation problems. The implicit proof naturally points to an explicit construction and was, in fact, the path taken by the authors to arrive at the explicit construction. In Section VIII-D we extend a noninteractive rate-distortion problem in [15] to an interactive problem, to construct an example where the one-message rate-distortion function can be arbitrarily large and the two-message sum-rate can be arbitrarily small.

\section{B. Implicit proof of the benefit of interaction}

Theorem 6: For the interactive rate-distortion problem where only terminal $B$ reproduces source sequence $\mathbf{X}$, there exists a distortion function $d$, a joint distribution $p_{X Y}$, and a distortion level $D$ for which $R_{\text {sum, }}^{A}\left(p_{X Y}, D\right)>$ $R_{\text {sum }, 2}^{B}\left(p_{X Y}, D\right)$.

Proof: According to Corollary [5, to prove Theorem 6, it is sufficient to show there exist $p_{X \mid Y}, d$, and $D$ for which $\rho_{1}^{A}\left(p_{X \mid Y} p_{Y}, D\right)$ is not concave with respect to $p_{Y}$. In particular, it is sufficient to show that there exist $p_{Y, 1}$

\footnotetext{
${ }^{7}$ In Section VIII the auxiliary variable in the one-message problem is denoted by $U$ and the auxiliary variables the two-message problems are denoted by $V_{1}, V_{2}$. The purpose is to avoid confusion of auxiliary variables for different problems.
} 
and $p_{Y, 2}$ such that

$$
\rho_{1}^{A}\left(p_{X \mid Y} \frac{p_{Y, 1}+p_{Y, 2}}{2}, D\right)<\frac{\rho_{1}^{A}\left(p_{X \mid Y} p_{Y, 1}, D\right)+\rho_{1}^{A}\left(p_{X \mid Y} p_{Y, 2}, D\right)}{2} .
$$

Let $\mathcal{X}=\mathcal{Y}=\{0,1\}$, and $\widehat{X}=\{0,1, e\}$. Let $d$ be the binary erasure distortion function, i.e., $d:\{0,1\} \times\{0, e, 1\} \rightarrow$ $\{0,1, \infty\}$ and for $i=0,1, d(i, i)=0, d(i, 1-i)=\infty$, and $d(i, e)=18$. Let $p_{Y, 1}(1)=1-p_{Y, 1}(0)=p_{Y, 2}(0)=1-p_{Y, 2}(1)=q$, i.e., $p_{Y, 1}=\operatorname{Bernoulli}(q)$ and $p_{Y, 2}=\operatorname{Bernoulli}(\bar{q})$. Let $p_{X \mid Y}$ be the conditional pmf of the binary symmetric channel with crossover probability $p$, i.e., $p_{X \mid Y}(1 \mid 0)=p_{X \mid Y}(0 \mid 1)=p$. Let $p_{Y}:=\left(p_{Y, 1}+p_{Y, 2}\right) / 2$ which is Bernoulli $(1 / 2)$. The joint distribution $p_{X Y}=p_{Y} p_{X \mid Y}$ is the joint pmf of a pair of doubly symmetric binary sources (DSBS) with parameter $p$, i.e., if $p_{x y}$ denotes $p_{X Y}(x, y)$, then $p_{00}=p_{11}=\bar{p} / 2$ and $p_{01}=p_{10}=p / 2$. For these choices of $p_{X \mid Y}, p_{Y, 1}, p_{Y, 2}, p_{Y}$, and $d$, we will analyze the left and right sides of (13) step by step through a sequence of propositions and establish the strict inequality for a suitable choice of $D$. The proofs of all the propositions are given in Appendix $\mathbb{E}$

- Left-side of (13): We have

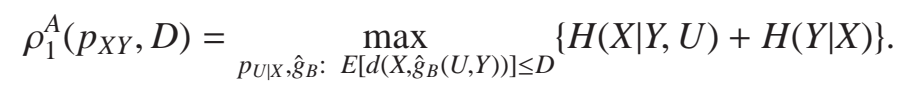

For the binary erasure distortion and a full-support joint source pmf taking values in binary alphabets, (14) simplifies to the expression given in Proposition 4

Proposition 4: If $\mathcal{X}=\mathcal{Y}=\{0,1\}, \operatorname{supp}\left(p_{X Y}\right)=\{0,1\}^{2}, d$ is the binary erasure distortion, and $D \in \mathbb{R}$, then $\rho_{1}=\max _{p_{U \mid X}}(H(X \mid Y, U)+H(Y \mid X))$, where $\mathcal{U}=\{0, e, 1\}$ and

$$
p_{U \mid X}(u \mid x)= \begin{cases}\alpha_{0 e}, & \text { if } x=0, u=e, \\ 1-\alpha_{0 e}, & \text { if } x=0, u=0, \\ \alpha_{1 e}, & \text { if } x=1, u=e, \\ 1-\alpha_{1 e}, & \text { if } x=1, u=1, \\ 0, & \text { otherwise, }\end{cases}
$$

where $\alpha_{0 e}, \alpha_{1 e} \in[0,1]$ satisfy $E[d(X, U)]=p_{X}(0) \alpha_{0 e}+p_{X}(1) \alpha_{1 e} \leq D$.

The expression for $\rho_{1}^{A}$ further simplifies to the one in Proposition 5 by using $p_{U \mid X}$ given by (15) in (14).

Proposition 5: If $\mathcal{X}=\mathcal{Y}=\{0,1\}, \operatorname{supp}\left(p_{X Y}\right)=\{0,1\}^{2}, d$ is the binary erasure distortion, and $D \in \mathbb{R}$, then

$$
\rho_{1}^{A}\left(p_{X Y}, D\right)=\max _{\substack{\alpha_{0 e}, \alpha_{1} \in[0,1]: \\ \phi\left(p_{X Y}, \alpha_{0 e}, \alpha_{1 e}\right) \leq D}} \psi\left(p_{X Y}, \alpha_{0 e}, \alpha_{1 e}\right)
$$

where

$$
\begin{aligned}
\psi\left(p_{X Y}, \alpha_{0 e}, \alpha_{1 e}\right) & \\
:= & \left(p_{00} \alpha_{0 e}+p_{10} \alpha_{1 e}\right) h_{2}\left(\frac{p_{00} \alpha_{0 e}}{p_{00} \alpha_{0 e}+p_{10} \alpha_{1 e}}\right) \\
& +\left(p_{01} \alpha_{0 e}+p_{11} \alpha_{1 e}\right) h_{2}\left(\frac{p_{01} \alpha_{0 e}}{p_{01} \alpha_{0 e}+p_{11} \alpha_{1 e}}\right) \\
& +\left(p_{00}+p_{01}\right) h_{2}\left(\frac{p_{00}}{p_{00}+p_{01}}\right)+\left(p_{11}+p_{10}\right) h_{2}\left(\frac{p_{11}}{p_{11}+p_{10}}\right),
\end{aligned}
$$

$\phi\left(p_{X Y}, \alpha_{0 e}, \alpha_{1 e}\right):=p_{X}(0) \alpha_{0 e}+p_{X}(1) \alpha_{1 e}$.

\footnotetext{
${ }^{8}$ Although the distortion function is assumed to take finite values in Section VII the results can be extended to some non-finite distortion measures including the binary erasure distortion. See [16, Problem 2.2.13].
} 
Finally, for a DSBS with parameter $p$ and the binary erasure distortion, $\rho_{1}^{A}$ reduces to the compact expression in Proposition 6

Proposition 6: If $d$ is the binary erasure distortion, $D \in[0,1]$, and $p_{X Y}$ is the joint pmf of a DSBS with parameter $p$, then

$$
\rho_{1}^{A}\left(p_{X Y}, D\right)=(1+D) h_{2}(p) .
$$

- Right-side of (13): Solving the rate reduction functionals in the right-side of (13) requires solving the maximization problem (16) for asymmetric distributions $p_{X \mid Y} p_{Y, 1}$ and $p_{X \mid Y} p_{Y, 2}$. Exactly solving this problem is cumbersome but it is easy to provide a lower bound for the maximum as follows.

Proposition 7: If $d$ is the binary erasure distortion, $p_{Y, 1}$ is $\operatorname{Bernoulli}(q), p_{Y, 2}$ is $\operatorname{Bernoulli}(\bar{q})$, and $p_{X \mid Y}$ is the conditional pmf of the binary symmetric channel with crossover probability $p$, then the inequality

$$
\frac{\rho_{1}^{A}\left(p_{X \mid Y} p_{Y, 1}, D\right)+\rho_{1}^{A}\left(p_{X \mid Y} p_{Y, 2}, D\right)}{2} \geq C\left(p, q, \alpha_{0 e}, 1\right)
$$

holds for $D=\eta\left(p, q, \alpha_{0 e}, 1\right)$, where

$$
\begin{aligned}
C\left(p, q, \alpha_{0 e}, \alpha_{1 e}\right) & :=\psi\left(p_{X \mid Y} p_{Y, 1}, \alpha_{0 e}, \alpha_{1 e}\right), \\
\eta\left(p, q, \alpha_{0 e}, \alpha_{1 e}\right) & :=\phi\left(p_{X \mid Y} p_{Y, 1}, \alpha_{0 e}, \alpha_{1 e}\right) .
\end{aligned}
$$

Remark 8: The rate-distortion tuple $\left(H(X \mid Y)+H(Y \mid X)-C\left(p, q, \alpha_{0 e}, 1\right), \eta\left(p, q, \alpha_{0 e}, 1\right)\right)$ is admissible for onemessage source coding for joint source pmf $p_{X \mid Y} p_{Y, 1}$ and corresponds to choosing $p_{U \mid X}$ given by (15) with $\alpha_{1 e}=1$ and the decoding function $g(u, y)=u$. Since this choice of $p_{U \mid X}$ and $\hat{g}_{B}$ may be suboptimal, $C\left(p, q, \alpha_{0 e}, 1\right)$ is only a lower bound for the rate reduction functional.

- Comparing left and right sides of (13): The left-side of (13) and the lower bound of the right-side of (13) can be compared as follows.

Proposition 8: Let $d$ be the binary erasure distortion, $p_{Y}$ be Bernoulli(1/2), and $p_{X \mid Y}$ be the binary symmetric channel with parameter $p$. For all $q \in(0,1 / 2)$ and all $\alpha_{0 e} \in(0,1)$, there exists $p \in(0,1)$ such that the strict inequality $\rho_{1}^{A}\left(p_{X Y}, D\right)<C\left(p, q, \alpha_{0 e}, 1\right)$ holds for $D=\eta\left(p, q, \alpha_{0 e}, 1\right)$.

Since the left-side of (13) is strictly less than a lower bound of the right-side of (13), the strict inequality (13) holds, which completes the proof of Theorem 6

\section{Explicit proof of the benefit of interaction}

Theorem 7 quantifies the multiplicative reduction in the sum-rate that is possible with two messages.

Theorem 7: If $d$ is the binary erasure distortion and $p_{X Y}$ the joint pmf of a DSBS with parameter $p$, then for all $L>0$ there exists an admissible two-message rate-distortion tuple $\left(R_{1}, R_{2}, D\right)$ such that $R_{\text {sum }, 1}^{A}\left(p_{X Y}, D\right) /\left(R_{1}+R_{2}\right)>L$ and $R_{1} / R_{2}<1 / L$.

Proof: The following single-letter characterization of $\mathcal{R} \mathcal{D}_{2}^{B}$ was established in [3]:

$$
\begin{aligned}
\mathcal{R D}_{2}^{B}=\{ & \left(R_{1}, R_{2}, D\right) \mid \exists p_{V_{1} \mid Y}, p_{V_{2} \mid X V_{1}}, \hat{g}_{B}, \text { s.t. } \\
& R_{1} \geq I\left(Y ; V_{1} \mid X\right), \\
& R_{2} \geq I\left(X ; V_{2} \mid Y, V_{1}\right), \\
& \left.\mathbb{E}\left[d\left(X, \hat{g}_{B}\left(V_{1}, V_{2}, Y\right)\right)\right] \leq D\right\},
\end{aligned}
$$


where $V_{1} \in \mathcal{V}_{1}$ and $V_{2} \in \mathcal{V}_{2}$ are auxiliary random variables with bounded alphabets, such that the Markov chains $V_{1}-Y-X$ and $V_{2}-\left(X, V_{1}\right)-Y$ hold, and $\hat{g}_{B}: \mathcal{V}_{1} \times \mathcal{V}_{2} \times \mathcal{Y} \rightarrow \widehat{X}$ is a deterministic single-letter decoding function.

We will explicitly construct $p_{V_{1} \mid Y}, p_{V_{2} \mid X V_{1}}$, and $\hat{g}_{B}$ which lead to an admissible tuple $\left(R_{1}, R_{2}, D\right)$. Let $p_{V_{1} \mid Y}$ be the conditional pmf of the binary symmetric channel with crossover probability $q$. Let the conditional distribution $p_{V_{2} \mid X V_{1}}\left(v_{2} \mid x, v_{1}\right)$ have the form described in Table $\square$ and let $\hat{g}_{B}\left(v_{1}, v_{2}, y\right):=v_{2}$.

TABLE I

CONDITIONAL Distribution $p_{V_{2} \mid X V_{1}}$

\begin{tabular}{|c|c|c|c|}
\hline$p_{V_{2} \mid X V_{1}}$ & $v_{2}=0$ & $v_{2}=e$ & $v_{2}=1$ \\
\hline$x=0, v_{1}=0$ & $1-\alpha$ & $\alpha$ & 0 \\
\hline$x=1, v_{1}=0$ & 0 & 1 & 0 \\
\hline$x=0, v_{1}=1$ & 0 & 1 & 0 \\
\hline$x=1, v_{1}=1$ & 0 & $\alpha$ & $1-\alpha$ \\
\hline
\end{tabular}

The corresponding rate-distortion tuple can be shown to satisfy the following property which is proved in Appendix 国

Proposition 9: Let $d$ be the binary erasure distortion and let $p_{X Y}$ be the joint pmf of a DSBS with parameter $p$. For $p_{V_{1} \mid Y}, p_{V_{2} \mid X V_{1}}$, and $\hat{g}_{B}$ as described above, and all $L>0$, there exist parameters $p, q, \alpha$ such that the twomessage rate-distortion tuple $\left(R_{1}, R_{2}, D\right)$ given by $R_{1}=I\left(Y ; V_{1} \mid X\right), R_{2}=I\left(X ; V_{2} \mid Y, V_{1}\right), D=E\left[d\left(X, V_{2}\right)\right]$ satisfies $R_{\text {sum }, 1}^{A}\left(p_{X Y}, D\right) /\left(R_{1}+R_{2}\right)>L$ and $R_{1} / R_{2}<1 / L$.

This completes the proof of Theorem 7 .

The conditional pmfs $p_{V_{1} \mid Y}$ and $p_{V_{2} \mid X V_{1}}$ in the proof of Theorem 7 are related to the conditional pmf $p_{U \mid X}$ in the proof of Theorem 6 as follows. Given $V_{1}=0$, the conditional distribution $p_{X Y V_{2} \mid V_{1}}\left(x, y, v_{2} \mid 0\right)=p_{Y, 1}(y) p_{X \mid Y}(x \mid y) p_{U \mid X}\left(v_{2} \mid x\right)$, where $p_{U \mid X}$ is given by (15) with $\alpha_{0 e}=\alpha$ and $\alpha_{1 e}=1$. Given $V_{1}=1$, the conditional distribution $p_{X Y V_{2} \mid V_{1}}\left(x, y, v_{2} \mid 1\right)=$ $p_{Y, 2}(y) p_{X \mid Y}(x \mid y) p_{U \mid X}\left(v_{2} \mid x\right)$, where $p_{U \mid X}$ is given by (15) with $\alpha_{1 e}=\alpha$ and $\alpha_{0 e}=1$. Conditioning on $V_{1}$, in effect, decomposes the two-message problem into two one-message problems that were analyzed in the proof of Theorem 6

\section{Example showing $R_{\text {sum }, 1}^{A}$ can be arbitrarily large and $R_{\text {sum }, 2}^{B}$ can be arbitrarily small}

In the example described in Section VIII-C, the multiplicative gain $R_{\text {sum }, 1}^{A} /\left(R_{1}+R_{2}\right)$ is shown to be arbitrarily large. The additive gain $R_{\text {sum }, 1}^{A}-\left(R_{1}+R_{2}\right)$, however, is not shown to be large. In this subsection we provide an example where $R_{\text {sum }, 1}^{A}$ can be arbitrarily large and the two-message sum-rate $\left(R_{1}+R_{2}\right)$ can be arbitrarily small, so that both $R_{\text {sum }, 1}^{A} /\left(R_{1}+R_{2}\right)$ and $R_{\text {sum }, 1}^{A}-\left(R_{1}+R_{2}\right)$ are arbitrarily large. Interestingly, $R_{1} / R_{2}$ can be arbitrarily small at the same time. The noninteractive one-message version of this example was provided in [15]. In this paper we extend it to an interactive problem to demonstrate the benefit of interaction.

To construct the example, we need to introduce the notion of a planar difference set used in [15]. Let $\mathcal{G}$ be an Abelian group. A subset $\mathcal{Z} \subseteq \mathcal{G}$ is a planar difference set of $\mathcal{G}$ if it is the largest possible subset such that for all non-zero $d \in \mathcal{G}$ the equation $z_{1}-z_{2}=d$ has exactly one solution for $z_{1}, z_{2} \in \mathcal{Z}$. Although not every Abelian group has a subset that is a planar different set, it is true that for any prime number $p$ and any positive integer $m$, a planar difference set of size $\alpha=p^{m}+1$ exists in the group of integers modulo $\left(\alpha^{2}-\alpha+1\right)$ (see [15]). Thus, there exist planar difference sets of arbitrarily large size.

For our example, we take $\mathcal{G}$ to be the Abelian group of integers with addition modulo $\left(\alpha^{2}-\alpha+1\right)$, where $\alpha=p^{m}+1$, $p$ prime and $m$ a positive integer. Let $\mathcal{Z}$ be a planar difference set of $\mathcal{G}$ of size $\alpha$. Let $(W, S, K) \sim p_{W S K}:=p_{W} p_{S \mid W} p_{K}$, 
where $p_{W}(2)=1-p_{W}(1):=\epsilon \in(0,1), p_{K}:=\operatorname{Unif}_{\mathcal{G}}, p_{S \mid W}(\cdot \mid 1):=\operatorname{Unif}_{\mathcal{Z}}$, and $p_{S \mid W}(\cdot \mid 2):=\operatorname{Unif}_{\mathcal{G}}$. Let $X:=(S+K)$ and $Y:=(K, W)$.

Let $\{(X(i), Y(i))\}_{i=1}^{n}$ be $n$ iid samples of a two-component discrete memoryless stationary source with joint pmf $p_{X Y} \cdot \mathbf{X}=(X(1), \ldots, X(n))$ is available at terminal $A$ and $\mathbf{Y}=(Y(1), \ldots, Y(n))$ is available at terminal $B$. Let $\mathbf{W}=(W(1), \ldots, W(n))$ denote the second component of source $\mathbf{Y} . B$ is required to reproduce $\mathbf{X}$ as $\widehat{\mathbf{X}}$. The distortion function is defined as follows.

$$
d(x, \hat{x})=\left\{\begin{array}{cl}
0, & \text { if }(x-\hat{x}) \in \mathcal{Z} \\
\infty, & \text { otherwise. }
\end{array}\right.
$$

The one-message Wyner-Ziv rate-distortion function for this problem was evaluated in [15] (see Equation (23) and the last but one displayed equation in [15]) and is given by

$$
\forall D>0, \quad R_{\text {sum }, 1}^{A}(D)=(1-\epsilon)\left(1-\frac{1}{|\mathcal{Z}|}\right) \log _{2}|\mathcal{Z}|+\epsilon\left(\log _{2}|\mathcal{G}|-\log _{2}|\mathcal{Z}|\right) .
$$

Since $|\mathcal{Z}|=\alpha$ and $|\mathcal{G}|=\alpha^{2}-\alpha+1$, we have

$$
R_{\text {sum }, 1}^{A}(D)=(1-\epsilon)\left(1-\frac{1}{\alpha}\right) \log _{2} \alpha+\epsilon \log _{2}\left(\alpha-1+\frac{1}{\alpha}\right) .
$$

Let us now consider a two-message interactive code with initial terminal $B$. In the first message, terminal $B$ sends $\mathbf{W}$ to $A$ at the rate $R_{1}=H(W)=h_{2}(\epsilon)$. For all the sample indices $i \in\{1, \ldots, n\}$ such that $W(i)=1$ (which implies $S(i) \in \mathcal{Z}$ ), terminal $A$ sends nothing and terminal $B$ generates $\widehat{X}(i)=K(i)$. Since for such sample indices $d(X(i), K(i))=d(S(i), 0)=0$ holds, the distortion at these samples is zero. For the remaining sample indices, $W(i)=$ 2, and terminal $A$ sends $X(i)$ completely at rate $H(X)=\log _{2}|\mathcal{G}|$, and terminal $B$ sets $\widehat{X}(i)=X(i)$. The distortion at these samples is also zero. The rate of the second message is $R_{2}=p_{W}(2) H(X)=\epsilon \log _{2}|\mathcal{G}|=\epsilon \log _{2}\left(\alpha^{2}-\alpha+1\right)$. Hence $\left(R_{1}, R_{2}, D\right)=\left(h_{2}(\epsilon), \epsilon \log _{2}\left(\alpha^{2}-\alpha+1\right), 0\right)$ is an admissible two-message rate-distortion tuple.

Now take $\epsilon=1 /(\log |\mathcal{G}|)^{2}=1 /\left(\log _{2}\left(\alpha^{2}-\alpha+1\right)\right)^{2}$ and consider the asymptotic behavior of $R_{\text {sum }, 1}^{A},\left(R_{1} / R_{2}\right),\left(R_{1}+R_{2}\right)$ as $\alpha$ increases. As $\alpha \rightarrow \infty$ we have

$$
\begin{aligned}
& R_{\text {sum }, 1}^{A}(D) \sim \log _{2} \alpha, \\
& \frac{R_{1}}{R_{2}}=\frac{h_{2}(\epsilon)}{\epsilon \log _{2}\left(\alpha^{2}-\alpha+1\right)} \sim \frac{\log _{2}(1 / \epsilon)}{\log _{2}\left(\alpha^{2}-\alpha+1\right)} \sim \frac{\log _{2} \log _{2} \alpha}{\log _{2} \alpha}, \\
& \left(R_{1}+R_{2}\right) \sim R_{2}=\epsilon \log _{2}\left(\alpha^{2}-\alpha+1\right)=\frac{1}{\log _{2}\left(\alpha^{2}-\alpha+1\right)} \sim \frac{1}{2 \log _{2} \alpha},
\end{aligned}
$$

where $f_{1}(\alpha) \sim f_{2}(\alpha)$ denotes $\lim _{\alpha \rightarrow \infty} f_{1}(\alpha) / f_{2}(\alpha)=1$. Thus we have proved the following theorem.

Theorem 8: There exists an interactive source reproduction problem, for which the following properties hold simultaneously: (i) the Wyner-Ziv rate-distortion function $R_{\text {sum }, 1}^{A}$ is arbitrarily large, (ii) the two-message sum-rate $\left(R_{1}+R_{2}\right)$ is arbitrarily small, and (iii) the two-message sum-rate can be split in a way that $\left(R_{1} / R_{2}\right)$ is arbitrarily small.

\section{Concluding Remarks}

In this work, we studied a two-terminal interactive function computation problem with alternating messages within the framework of distributed block source coding theory. We developed a new limit-free single-letter characterization of the infinite-message sum-rate-distortion function by viewing it as a functional of the joint source distribution 
and distortion levels and studying its convex-geometric properties. This led to an optimality test for any achievable rate-distortion functional and an iterative algorithm for computing the infinite-message limit. This allowed us to fully characterize the limit for computing the Boolean AND function of independent at one or both terminals and to construct the first examples that demonstrate that the Wyner-Ziv rate-distortion function can be strictly improved using two messages.

Several questions remain unresolved. First, it is unclear if a limit-free characterization can be developed for a single joint source pmf and a specified pair of distortion levels. The current characterization is for a whole family of pmfs that is closed under marginal-perturbations. We have seen that for some pmfs, the infinite-message limit is reached using a finite number of messages. Is there a simple way to test this for a given pmf? Is there a simple way to estimate the number of messages needed to reach the limit? For computing the Boolean AND function of independent sources at one or both terminals, we used a particular infinite-sequence of auxiliary random variables which, for some pmfs, suggests the need for an infinite number of infinitesimal-rate messages. Is this fundamentally necessary or is it an artifact of our particular construction? Algorithmic issues were not the focus of this work. A formal complexity-analysis of the iterative algorithm would be an interesting direction of research. Here we established pointwise convergence of the algorithm but did not characterize the rate of convergence. There is some empirical evidence to suggest that the convergence is uniform and quadratic but it is unclear if this is true in general. Another important question that is only empirically addressed here is the impact of the discretization step-size in a computer implementation of the iterative algorithm.

The success of the approach described in this paper hinges on the availability of a finite-message single-letter characterization. A fascinating open question is whether the functional characterization can be arrived at from firstprinciples without access to the finite-message single letter characterization. If so, then it may pave the way for analyzing other multi-terminal interactive source coding problems where single-letter characterizations are rarely available. Our present approach can be extended (with suitable modifications) to handle the so-called collocated networks [9] where source nodes observe independent sources and make a sequence of noiseless broadcasts that are heard by all other nodes and a sink node that wishes to compute a multivariable function of all the sources. More general network problems seem daunting at this time. One approach to make progress would be to develop "interactive cutset bounds" and "collocated network bounds" as in [9]. Connections to entropic regions in network coding ( [10], [11, Chapter 21]) are also worth exploring.

\section{ACKNOWLEDGMENT}

The authors would like to thank Prof. David Castañón for discussions related to marginal-perturbations-closed families of pmfs, Dr. Vinod Prabhakaran for introducing them to the unresolved question in [3], and Prof. Ram Zamir for introducing them to the example in Section VIII-D and for suggesting that it might provide another example where the Wyner-Ziv rate-distortion function can be strictly improved.

\section{ApPendix A}

Proof of Lemma 1

First we define two collections of conditional pmfs for convenience:

$$
\mathcal{P}_{\text {ent }, t}\left(p_{X Y}, f_{A}, f_{B}\right):=\left\{p_{U^{t} \mid X Y}: \text { condition (i) (conditional entropy constraint) of Fact } 1 \text { holds }\right\},
$$


$\mathcal{P}_{m c, t}^{A}:=\left\{p_{U^{t} \mid X Y}\right.$ : conditions (ii) and (iii) (Markov chains and cardinality bounds) of Fact 1 hold $\}$.

Then we have $\mathcal{P}_{t}^{A}\left(p_{X Y}, f_{A}, f_{B}\right):=\mathcal{P}_{m c, t}^{A} \cap \mathcal{P}_{\text {ent,t }}\left(p_{X Y}, f_{A}, f_{B}\right)$.

(i) For all $t \in \mathbb{Z}^{+}$and all $p_{X Y} \in \mathcal{P}_{X Y}$, we have

$$
\begin{aligned}
& \rho_{t}^{A}\left(p_{X Y}\right)=\max _{p_{U^{t} \mid X Y} \in \mathcal{P}_{t}^{A}\left(p_{X Y}\right)}\left\{H\left(X \mid Y, U^{t}\right)+H\left(Y \mid X, U^{t}\right)\right\}
\end{aligned}
$$

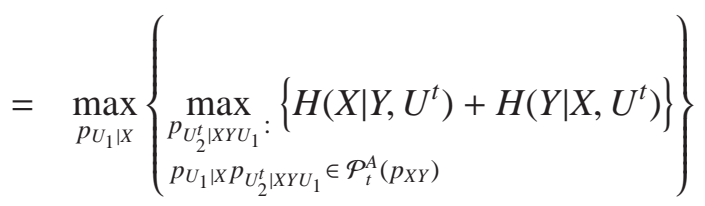

$$
\begin{aligned}
& \stackrel{(a)}{=} \max _{p_{U_{1}} \mid X}\left\{\sum_{u_{1} \in \operatorname{supp}\left(p_{U_{1}}\right)} p_{U_{1}}\left(u_{1}\right)\left\{\max _{\substack{p_{U_{2}^{t} \mid X Y U_{1}}\left(\cdot \cdot \cdot, ; u_{1}\right): \\
p_{U_{1}\left|X p_{U_{2}^{t}}\right| X Y U_{1}} \in \mathcal{P}_{t}^{A}\left(p_{X Y \mid U_{1}}\left(\cdot, \mid u_{1}\right)\right)}}\left\{H\left(X \mid Y, U_{2}^{t}, U_{1}=u_{1}\right)+H\left(Y \mid X, U_{2}^{t}, U_{1}=u_{1}\right)\right\}\right\}\right. \\
& \stackrel{(b)}{=} \max _{p_{U_{1} \mid X}}\left\{\sum_{u_{1} \in \operatorname{supp}\left(p_{U_{1}}\right)} p_{U_{1}}\left(u_{1}\right) \rho_{t-1}^{B}\left(p_{X Y \mid U_{1}}\left(\cdot, \cdot \mid u_{1}\right)\right)\right\} \text {. }
\end{aligned}
$$

Step (a) follows from the "law of total conditional entropy" with the additional observation that conditioned on $U_{1}=u_{1},\left(H\left(X \mid Y, U_{2}^{t}, U_{1}=u_{1}\right)+H\left(Y \mid X, U_{2}^{t}, U_{1}=u_{1}\right)\right)$ only depends on $p_{U_{2}^{t} \mid X Y U_{1}}\left(\cdot \mid \cdot, \cdot, u_{1}\right)$. Step (b) is due to the observation that for a fixed $p_{U_{1} \mid X}$, conditioned on $U_{1}=u_{1}$, (i) $p_{U_{1} \mid X} p_{U_{2}^{t} \mid X Y U_{1}} \in \mathcal{P}_{m c, t}^{A}$ iff $p_{U_{2}^{t} \mid X Y U_{1}} \in \mathcal{P}_{m c, t-1}^{B}$ and (ii) $p_{U_{1} \mid X} p_{U_{2}^{t} \mid X Y U_{1}} \in \mathcal{P}_{\text {ent, } t}\left(p_{X Y, u_{1}}, f_{A}, f_{B}\right)$, where $p_{X Y, u_{1}}:=p_{X Y \mid U_{1}}\left(\cdot, \cdot \mid u_{1}\right)$, iff $p_{U_{2}^{t} \mid X Y U_{1}} \in \mathcal{P}_{\text {ent }, t-1}\left(p_{X Y, u_{1}}, f_{A}, f_{B}\right)$. Therefore, $p_{U_{1} \mid X} p_{U_{2}^{t} \mid X Y U_{1}} \in \mathcal{P}_{t}^{A}\left(p_{X Y, u_{1}}\right)$ iff $p_{U_{2}^{t} \mid X Y U_{1}} \in \mathcal{P}_{t-1}^{B}\left(p_{X Y, u_{1}}\right)$.

(ii) For an arbitrary $q_{X Y} \in \mathcal{P}_{X Y}$, consider two arbitrary joint pmfs $p_{X Y, 1}, p_{X Y, 0} \in \mathcal{P}_{Y \mid X}\left(q_{X Y}\right)$. For every $\lambda \in(0,1)$, let $p_{X Y, \lambda}:=\lambda p_{X Y, 1}+\bar{\lambda} p_{X Y, 0}$. Due to Remark 2(i), $p_{X Y, \lambda} \in \mathcal{P}_{Y \mid X}\left(q_{X Y}\right)$. We need to show that $\rho_{t}^{A}\left(p_{X Y, \lambda}\right) \geq \lambda \rho_{t}^{A}\left(p_{X Y, 1}\right)+$ $\bar{\lambda} \rho_{t}^{A}\left(p_{X Y, 0}\right)$. For $i=0,1$, let $p_{U_{1} \mid X, i}$ be the conditional pmf that maximizes the objective function in (5) for source pmf $p_{X Y, i}$. Let $p_{U_{1}, i}$ and $p_{X Y \mid U_{1}, i}$ denote, respectively, the $U_{1}$-marginal and conditional pmfs of $p_{X Y, i} p_{U_{1} \mid X, i}$. Thus we have $\rho_{t}^{A}\left(p_{X Y, i}\right)=\sum_{u_{1}} p_{U_{1}, i}\left(u_{1}\right) \rho_{t-1}^{B}\left(p_{X Y \mid U_{1}, i}\left(\cdot, \cdot \mid u_{1}\right)\right)$. Define a new auxiliary variable $V \in \mathcal{U}_{1} \times\{0,1\}$ with pmf $p_{V}\left(u_{1}, 1\right):=\lambda p_{U_{1}, 1}\left(u_{1}\right)$ and $p_{V}\left(u_{1}, 0\right):=\bar{\lambda} p_{U_{1}, 0}\left(u_{1}\right)$. Let $p_{X Y \mid V}\left(\cdot, \cdot \mid u_{1}, i\right):=p_{X Y \mid U_{1}, i}\left(\cdot, \cdot \mid u_{1}\right)$. Then the $X Y$-marginal pmf of $p_{V} p_{X Y \mid V}$ is $p_{X Y, \lambda}$. We have,

$$
\begin{aligned}
\lambda \rho_{t}^{A}\left(p_{X Y, 1}\right)+\bar{\lambda} \rho_{t}^{A}\left(p_{X Y, 0}\right) & =\lambda \sum_{u_{1}} p_{U_{1}, 1}\left(u_{1}\right) \rho_{t-1}^{B}\left(p_{X Y \mid U_{1}, 1}\left(\cdot, \cdot \mid u_{1}\right)\right)+\bar{\lambda} \sum_{u_{1}} p_{U_{1}, 0}\left(u_{1}\right) \rho_{t-1}^{B}\left(p_{X Y \mid U_{1}, 0}\left(\cdot, \cdot \mid u_{1}\right)\right) \\
& =\sum_{u_{1}, i} p_{V}\left(u_{1}, i\right) \rho_{t-1}^{B}\left(p_{X Y \mid V}\left(\cdot, \cdot \mid u_{1}, i\right)\right) \\
& \leq \rho_{t}^{A}\left(p_{X Y, \lambda}\right),
\end{aligned}
$$

where the last step is because (5) holds for source pmf $p_{X Y, \lambda}$.

(iii) First we make the following observation.

Remark 9: For all $u_{1} \in \operatorname{supp}\left(p_{U_{1}}\right), p_{X Y \mid U_{1}}\left(\cdot, \cdot \mid u_{1}\right) \in \mathcal{P}_{Y \mid X}\left(p_{X Y}\right)$. This is confirmed by noting that since $Y-X-U_{1}$ is a Markov chain, $\forall\left(x, u_{1}\right) \in \operatorname{supp}\left(p_{X} p_{U_{1} \mid X}\right)$ we have $p_{Y \mid X U_{1}}\left(y \mid x, u_{1}\right)=p_{Y \mid X}(y \mid x)$ (see the paragraph after Definition 1). 
For all $p_{X Y} \in \mathcal{P}_{Y \mid X}\left(q_{X Y}\right)$, we have

$$
\begin{aligned}
\rho_{t}^{A}\left(p_{X Y}\right) & \stackrel{(c)}{=} \max _{p_{U_{1} \mid X}}\left\{\sum_{u_{1}} p_{U_{1}}\left(u_{1}\right) \rho_{t-1}^{B}\left(p_{X Y \mid U_{1}}\left(\cdot, \cdot \mid u_{1}\right)\right)\right\} \\
& \stackrel{(d)}{\leq} \max _{p_{U_{1} \mid X}}\left\{\sum_{u_{1}} p_{U_{1}}\left(u_{1}\right) \rho\left(p_{X Y \mid U_{1}}\left(\cdot, \cdot \mid u_{1}\right)\right)\right\} \\
& \stackrel{(e)}{\leq} \rho\left(p_{X Y}\right),
\end{aligned}
$$

where the equality (c) follows from (5), the inequality (d) is true because $\rho_{t-1}^{B}\left(p_{X Y}\right) \leq \rho\left(p_{X Y}\right)$, and the final inequality (e) is true due to the following reasons (i) $p_{X Y \mid U_{1}}\left(\cdot, \cdot \mid u_{1}\right) \in \mathcal{P}_{Y \mid X}\left(p_{X Y}\right) \subseteq \mathcal{P}_{Y \mid X}\left(q_{X Y}\right)$ due to Remarks 2 and 9 , (ii) $\rho$ is concave on $\mathcal{P}_{Y \mid X}\left(q_{X Y}\right)$, and (3) Jensen's inequality.

(iv) This part is proved by reversing the roles of terminal $A$ and $B$ in parts (i) - (iii).

\section{APPENDIX B}

Proof of Proposition 3

We first prove part (i) of the proposition. Let $\left(X_{[0]}, Y_{[0]}\right)$ and $\left(X_{[1]}, Y_{[1]}\right)$ be independent random tuples with $\left(X_{[\theta]}, Y_{[\theta]}\right) \sim p_{X Y_{[\theta]}} \in \Delta(\mathcal{X} \times \mathcal{Y})$, for $\theta=0,1$. Let $\Theta \sim \operatorname{Ber}(\lambda), \lambda \in(0,1)$, be independent of $\left(X_{[0]}, Y_{[0]}\right)$ and $\left(X_{[1]}, Y_{[1]}\right)$. Then $\left(X_{[\Theta]}, Y_{[\Theta]}\right)$ has the mixture pmf: $\lambda p_{X Y_{[1]}}+(1-\lambda) p_{X Y_{[0]}}=: p_{X Y_{[\lambda]}}$.

Due to (1), for any $t$, we have

$$
\begin{aligned}
& R_{\text {sum }, t}^{A}\left(p_{X Y_{[\lambda]}}\right)=\min _{p_{U^{t} \mid X Y_{[\Theta]}} \in \mathcal{P}_{t}^{A}\left(p_{X Y_{[\lambda]}}\right)} I\left(X_{[\Theta]} ; U^{t} \mid Y_{[\Theta]}\right)+I\left(Y_{[\Theta]} ; U^{t} \mid X_{[\Theta]}\right) \\
& \stackrel{(a)}{=} \min _{p_{U^{t} \mid X Y_{[\Theta]}} \in \mathcal{P}_{t}^{A}\left(p_{X Y_{[X]}}\right)} I\left(X_{[\Theta]}, \Theta ; U^{t} \mid Y_{[\Theta]}\right)+I\left(Y_{[\Theta]}, \Theta ; U^{t} \mid X_{[\Theta]}\right) \\
& \geq \min _{p_{U^{t} \mid X Y_{[\Theta]}} \in \mathcal{P}_{t}^{A}\left(p_{X Y_{[X]}}\right)} I\left(X_{[\Theta]} ; U^{t} \mid Y_{[\Theta]}, \Theta\right)+I\left(Y_{[\Theta]} ; U^{t} \mid X_{[\Theta]}, \Theta\right) \\
& \geq \lambda\left(\min _{p_{U^{t} \mid X Y_{[\Theta]}} \in \mathcal{P}_{t}^{A}\left(p_{X Y_{[]]}}\right)} I\left(X_{[\Theta]} ; U^{t} \mid Y_{[\Theta]}, \Theta=1\right)+I\left(Y_{[\Theta]} ; U^{t} \mid X_{[\Theta]}, \Theta=1\right)\right) \\
& +(1-\lambda)\left(\min _{p_{U^{t} \mid X Y_{[\Theta]}} \in \mathcal{P}_{t}^{A}\left(p_{X Y_{[]]}}\right)} I\left(X_{[\Theta]} ; U^{t} \mid Y_{[\Theta]}, \Theta=0\right)+I\left(Y_{[\Theta]} ; U^{t} \mid X_{[\Theta]}, \Theta=0\right)\right) \\
& \stackrel{(b)}{\geq} \lambda\left(\min _{p_{U^{t} \mid X Y_{[\Theta]}} \in \mathcal{P}_{t}^{A}\left(p_{X Y_{[1]}}\right)} I\left(X_{[\Theta]} ; U^{t} \mid Y_{[\Theta]}, \Theta=1\right)+I\left(Y_{[\Theta]} ; U^{t} \mid X_{[\Theta]}, \Theta=1\right)\right) \\
& +(1-\lambda)\left(\min _{p_{U^{t} \mid X Y_{[\Theta]}} \in \mathcal{P}_{t}^{A}\left(p_{\left.X Y_{[0]}\right]}\right)} I\left(X_{[\Theta]} ; U^{t} \mid Y_{[\Theta]}, \Theta=0\right)+I\left(Y_{[\Theta]} ; U^{t} \mid X_{[\Theta]}, \Theta=0\right)\right) \\
& =\lambda R_{\text {sum }, t}^{A}\left(p_{X Y_{[1]}}\right)+(1-\lambda) R_{\text {sum }, t}^{A}\left(p_{X Y_{[0]}}\right) .
\end{aligned}
$$

Step (a) is because $\Theta-\left(X_{[\Theta]}, Y_{[\Theta]}\right)-U^{t}$ forms a Markov chain. Step (b) is because $\mathcal{P}_{t}^{A}\left(p_{X Y_{[]]}}\right) \subseteq \mathcal{P}_{t}^{A}\left(p_{X Y_{[1]}}\right) \cap \mathcal{P}_{t}^{A}\left(p_{X Y_{[0]}}\right)$, which is due to the following three reasons. (i) $H\left(f_{A}\left(X_{[\Theta]}, Y_{[\Theta]}\right) \mid U^{t}\right)=0$ implies $H\left(f_{A}\left(X_{[\Theta]}, Y_{[\Theta]}\right) \mid U^{t}, \Theta\right)=0$, which in turn implies $H\left(f_{A}\left(X_{[\Theta]}, Y_{[\Theta]}\right) \mid U^{t}, \Theta=\theta\right)=0$ for $\theta=0,1$. The same property holds if $f_{A}$ is replaced by $f_{B}$. (ii) The Markov chain constraints induce the following factorization structure on the conditional pmf of $U^{t}$ given $X^{m}$ : 
$p_{U^{t} \mid X Y}\left(u^{t} \mid x, y\right)=p_{U_{1} \mid X}\left(u_{1} \mid x\right) \cdot p_{U_{2} \mid Y U_{1}}\left(u_{2} \mid y, u_{1}\right) \cdot p_{U_{3} \mid X U^{2}}\left(u_{3} \mid x, u^{2}\right) \ldots$, which is common for all source pmfs. (iii) The cardinality bounds are common for all source pmfs.

Part (ii) of the proposition immediately follows from part (i) by taking the limit $t \rightarrow \infty$.

APPENDIX C

Achievability of $R^{*}$ in Section $\nabla-B$

The achievability proof of $R^{*}(p, q)$ for $(p, q) \in(0,1 / 2]^{2}$ uses the same technique that was used in [1, Sec. IV.F]. If $R^{*}(p, q)$ can be shown to be an achievable sum-rate for $(p, q) \in(0,1 / 2]^{2}$, then $R^{*}(1-p, q)$ will be an achievable sum-rate for $(p, q) \in[1 / 2,1) \times(0,1 / 2]$. This is because when $p \geq 1 / 2, X^{c} \sim \operatorname{Ber}(1-p)$ (with $\left.(1-p) \leq 1 / 2\right)$ so that an achievable coding scheme for $(p, q) \in(0,1 / 2]^{2}$ can be used to enable $B$ to compute $\mathbf{X}^{c} \wedge \mathbf{Y}$ and therefore also $\mathbf{X} \wedge \mathbf{Y}=\left(\mathbf{X}^{c} \wedge \mathbf{Y}\right)^{c} \wedge \mathbf{Y}$. For $1 / 2 \leq q \leq 1$, the rate $H(X)=h_{2}(p)$ is an achievable sum-rate because it corresponds to a coding scheme in which $\mathbf{X}$ is completely reproduced at $B$ by sending a single message of rate $h_{2}(p)$ from $A$ to $B$. We will now show that $R^{*}(p, q)$ is an achievable sum-rate for $(p, q) \in(0,1 / 2]^{2}$. Since we are interested in the limit $t \rightarrow \infty$, it is sufficient to consider only even-valued $t$.

The proof of achievability of $R^{*}(p, q)$ is based on random coding and random binning arguments [17]. Choosing the auxiliary random variables $U^{t}$ in (1) plays a central role in the generation of random codebooks in random coding, and in determining the sizes of bins in random binning. As $t \rightarrow \infty$, an unbounded number of auxiliary random variables need to be chosen. In the next paragraph we describe a systematic procedure to construct $U^{t}$ which ensures that (i) the constructed $U^{t}$ satisfy the requirements in Fact 1 even when $t$ grows without bound, and (ii) the achievable sum-rate converges as $t \rightarrow \infty$.

Our construction of $U^{t}$ is based upon two extra auxiliary random variables $V_{x}$ and $V_{y}$. Let $\left(V_{x}, V_{y}\right) \sim \mathrm{Unif}_{[0,1]^{2}}$ be real auxiliary random variables and define $X:=1_{[1-p, 1]}\left(V_{x}\right)$ and $Y:=1_{[1-q, 1]}\left(V_{y}\right)$. Then $p_{X Y}$ is indistinguishable from the joint source pmf, i.e., $p_{X}(1)=1-p_{X}(0)=p, p_{Y}(1)=1-p_{Y}(0)=q$ and $X \Perp Y$. We will interpret the symbols 0 and 1 as real zero and real one respectively as needed. This interpretation will allow us to express Boolean arithmetic in terms of real arithmetic, e.g., $X \wedge Y$ (Boolean AND) $=X Y$ (real multiplication). Define a rate-allocation curve $\Gamma$ parametrically by $\Gamma:=\{(\alpha(s), \beta(s)), 0 \leq s \leq 1\}$ where $\alpha$ and $\beta$ are real, nondecreasing, absolutely continuous functions with $\alpha(0)=\beta(0)=0, \alpha(1)=(1-p)$, and $\beta(1) \in[0,1-q]$. We note that in [1, Sec. IV.F] where the AND function was required to be computed at both terminals rather than at only terminal $B, \Gamma$ was required to satisfy a different condition $\beta(1)=(1-q)$ and this lead to a different admissible sum-rate expression. The significance of $\Gamma$ will become clear later. Now choose a partition of $[0,1], 0=s_{0}<s_{1}<\ldots<s_{t / 2-1}<s_{t / 2}=1$, such that $\max _{i=1, \ldots, t / 2}\left(s_{i}-s_{i-1}\right)<\Delta_{t}$. For $i=1, \ldots, t / 2$, define $t$ auxiliary random variables as follows,

$$
U_{2 i-1}:=1_{\left[\alpha\left(s_{i}\right), 1\right] \times\left[\beta\left(s_{i-1}\right), 1\right]}\left(V_{x}, V_{y}\right), U_{2 i}:=1_{\left[\alpha\left(s_{i}\right), 1\right] \times\left[\beta\left(s_{i}\right), 1\right]}\left(V_{x}, V_{y}\right) .
$$

In Figure $5 \mathrm{a}),\left(V_{x}, V_{y}\right)$ is uniformly distributed on the unit square and $U^{t}$ are defined to be 1 in rectangular regions which are nested. The following properties can be verified:

$P 1: U_{1} \geq U_{2} \geq \ldots \geq U_{t}$.

$P 2: \quad p_{U^{t} \mid X Y}$ satisfies the conditional entropy constraint in Fact1, that is, $H\left(X \wedge Y \mid Y, U^{t}\right)=0$ : since $U_{t}=1_{[1-p, 1] \times[\beta(1), 1]}\left(V_{x}, V_{y}\right)$ and $Y=1_{[1-q, 1]}\left(V_{y}\right)$. Therefore $U_{t} \wedge Y=1_{[1-p, 1] \times[1-q, 1]}\left(V_{x}, V_{y}\right)=X \wedge Y$.

P3: $p_{U^{t} \mid X Y}$ satisfies the Markov chain constraints in Fact 1] for example, consider $U_{2 i}-\left(Y, U^{2 i-1}\right)-X$. $U_{2 i-1}=0 \Rightarrow$ $U_{2 i}=0$ and the Markov chain holds. $U_{2 i-1}=Y=1 \Rightarrow\left(V_{x}, V_{y}\right) \in\left[\alpha\left(s_{i}\right), 1\right] \times[1-q, 1] \Rightarrow U_{2 i}=1$ and the Markov chain holds. Given $U_{2 i-1}=1, Y=0,\left(V_{x}, V_{y}\right) \sim \operatorname{Unif}_{\left[\alpha\left(s_{i}\right), 1\right] \times\left[\beta\left(s_{i-1}\right), 1-q\right]} \Rightarrow V_{x}$ and $V_{y}$ are conditionally 


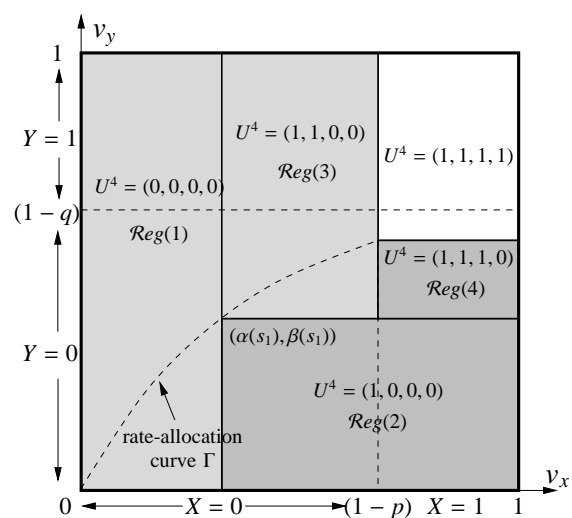

(a)

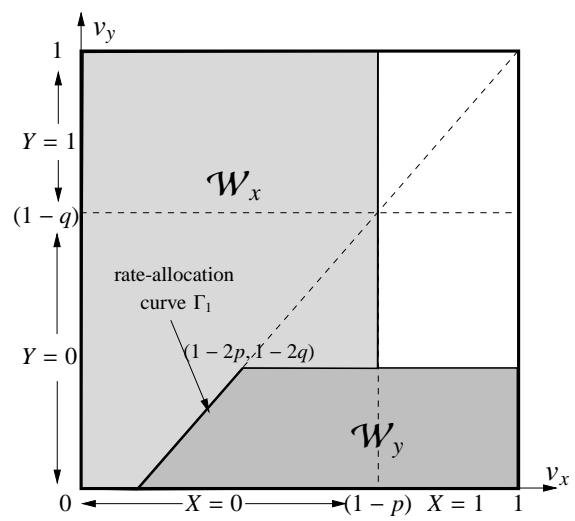

(c)

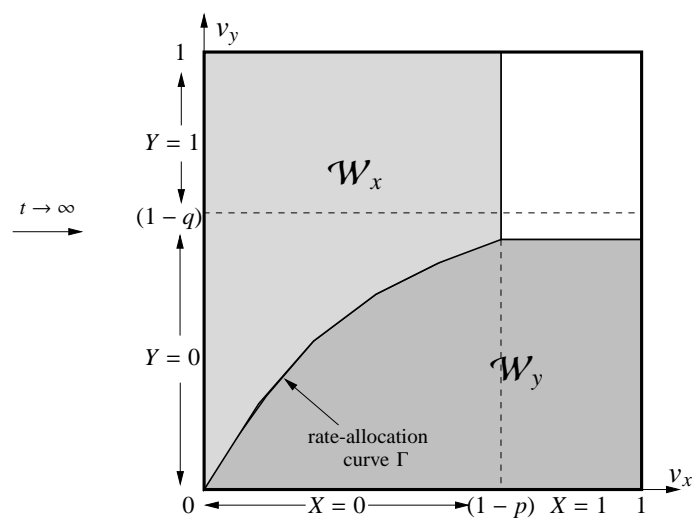

(b)

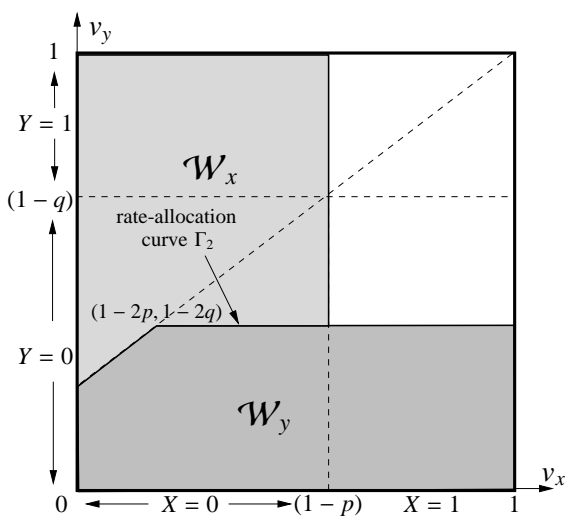

(d)

Fig. 5. (a) 4-message interactive code (b) $\infty$-message interactive code (c) $\infty$-message interactive code for $0<p \leq q \leq 1 / 2$ with rate-allocation curve $\Gamma_{1}(d) \infty$-message interactive code for $0<q \leq p \leq 1 / 2$ with rate-allocation curve $\Gamma_{2}$.

independent. Thus $X \Perp U_{2 i} \mid\left(U_{2 i-1}=1, Y=0\right)$ because $X$ is a function of only $V_{x}$ and $U_{2 i}$ is a function of only $V_{y}$ upon conditioning. So the Markov chain $U_{2 i}-\left(Y, U^{2 i-1}\right)-X$ holds in all situations.

P4: $\left(Y, U_{2 i}\right) \Perp X \mid U_{2 i-1}=1$ : this can be proved by the same method as in $P 3$.

$P 2$ and $P 3$ show that $p_{U^{t} \mid X Y} \in \mathcal{P}_{t}^{A}\left(p_{X Y}\right)$. 
For $i=1, \ldots, t / 2$, the $(2 i)$-th rate is given by

$$
\begin{aligned}
& I\left(Y ; U_{2 i} \mid X, U^{2 i-1}\right)= \\
& \stackrel{P 1}{=} I\left(Y ; U_{2 i} \mid X, U_{2 i-1}=1\right) p_{U_{2 i-1}}(1) \\
& \stackrel{P 4}{=} I\left(Y ; U_{2 i} \mid U_{2 i-1}=1\right) p_{U_{2 i-1}}(1) \\
&= H\left(Y \mid U_{2 i-1}=1\right) p_{U_{2 i-1}}(1)-H\left(Y \mid U_{2 i}, U_{2 i-1}=1\right) p_{U_{2 i-1}}(1) \\
& \stackrel{(a)}{=} H\left(Y \mid U_{2 i-1}=1\right) p_{U_{2 i-1}}(1)-H\left(Y \mid U_{2 i}=1\right) p_{U_{2 i}}(1) \\
&=\left(1-\alpha\left(s_{i}\right)\right)\left(\left(1-\beta\left(s_{i-1}\right)\right) h_{2}\left(\frac{q}{1-\beta\left(s_{i-1}\right)}\right)\right. \\
&\left.-\left(1-\beta\left(s_{i}\right)\right) h_{2}\left(\frac{q}{1-\beta\left(s_{i}\right)}\right)\right) \\
& \stackrel{(b)}{=}\left(1-\alpha\left(s_{i}\right)\right) \int_{\beta\left(s_{i-1}\right)}^{\beta\left(s_{i}\right)} \log _{2}\left(\frac{1-v_{y}}{1-q-v_{y}}\right) d v_{y} \\
&= \iint_{\left[\alpha\left(s_{i}\right), 1\right] \times\left[\beta\left(s_{i-1}\right), \beta\left(s_{i}\right)\right]} w_{y}\left(v_{y}, q\right) d v_{x} d v_{y},
\end{aligned}
$$

where step (a) is due to property $P 4$ and because $\left(U_{2 i-1}, U_{2 i}\right)=(1,0) \Rightarrow Y=0$, hence $H\left(Y \mid U_{2 i}, U_{2 i-1}=1\right) p_{U_{2 i-1}}(1)=$ $H\left(Y \mid U_{2 i}=1, U_{2 i-1}=1\right) p_{U_{2 i}, U_{2 i-1}}(1,1) \stackrel{P 1}{=} H\left(Y \mid U_{2 i}=1\right) p_{U_{2 i}}(1)$, and step (b) is because

$$
\frac{\partial}{\partial v_{y}}\left(-\left(1-v_{y}\right) h_{2}\left(\frac{q}{1-v_{y}}\right)\right)=\log _{2}\left(\frac{1-v_{y}}{1-q-v_{y}}\right)=: w_{y}\left(v_{y}, q\right) \text {. }
$$

The $2 i$-th rate can thus be expressed as a 2-D integral of a weight function $w_{y}$ over the rectangular region $\operatorname{Reg}(2 i):=$ $\left[\alpha\left(s_{i}\right), 1\right] \times\left[\beta\left(s_{i-1}\right), \beta\left(s_{i}\right)\right]$ (a horizontal bar in Figure $5(\mathrm{a})$ ). Therefore, the sum of rates of all messages sent from terminal $B$ to terminal $A$ is the integral of $w_{y}$ over the union of all the corresponding horizontal bars in Figure 5 (a). Similarly, the sum of rates of all messages sent from terminal $A$ to terminal $B$ can be expressed as the integral of another weight function $w_{x}\left(v_{x}, p\right):=\log _{2}\left(\left(1-v_{x}\right) /\left(1-p-v_{x}\right)\right)$ over the union of all the vertical bars in Figure 5 a).

Now let $t \rightarrow \infty$ such that $\Delta_{t} \rightarrow 0$. Since $\alpha$ and $\beta$ are absolutely continuous, $\left(\alpha\left(s_{i}\right)-\alpha\left(s_{i-1}\right)\right) \rightarrow 0$ and $\left(\beta\left(s_{i}\right)-\right.$ $\left.\beta\left(s_{i-1}\right)\right) \rightarrow 0$. The union of the horizontal (resp. vertical bars) in Figure 5 (a) tends to the region $\mathcal{W}_{y}\left(\right.$ resp. $\mathcal{W}_{x}$ ) in Figure 5 (b). Hence an achievable infinite-message sum-rate given by

$$
\iint_{\mathcal{W}_{x}} w_{x}\left(v_{x}, p\right) d v_{x} d v_{y}+\iint_{\mathcal{W}_{y}} w_{y}\left(v_{y}, q\right) d v_{x} d v_{y}
$$

depends on only the rate-allocation curve $\Gamma$ which coordinates the progress of source descriptions at $A$ and $B$. When $0<p \leq q \leq 1 / 2$, choose $\Gamma=\Gamma_{1}$ to be the piecewise linear curve connecting $(0,0),(1-p / q, 0),(1-2 p, 1-2 q)$, and $(1-p, 1-2 q)$ in that order (see Figure 5(c)). When $0<q \leq p \leq 1 / 2$, choose $\Gamma=\Gamma_{2}$ to be the piecewise linear curve connecting $(0,0),(0,1-q / p),(1-2 p, 1-2 q)$, and $(1-p, 1-2 q)$ in that order (see Figure 5 (d)). For these two choices of the rate-allocation curve, (C.1) can be evaluated in closed form and is given by the expressions in the first two cases of (7), which completes the proof.

Remark 10: The two curves $\Gamma_{1}$ and $\Gamma_{2}$ were specifically chosen to minimize the value of (C.1). The (nontrivial) proof of this fact is omitted because it is not needed to show that $R^{*}$ in 7 is an achievable sum-rate. 
Proof of Lemma 2

(i) For all $t \in \mathbb{Z}^{+}$and all $p_{X Y} \in \mathcal{P}_{X Y}$, we have

$$
\begin{aligned}
& \rho_{t}^{A}\left(p_{X Y}, \mathbf{D}\right) \\
& =\max _{\left(p_{U^{t} \mid X Y}, \hat{g}_{A}, \hat{g}_{B}\right) \in \mathcal{P}_{t}^{A}\left(p_{X Y}, \mathbf{D}\right)}\left\{H\left(X \mid Y, U^{t}\right)+H\left(Y \mid X, U^{t}\right)\right\}
\end{aligned}
$$

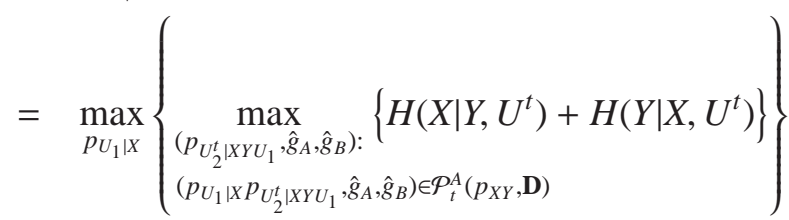

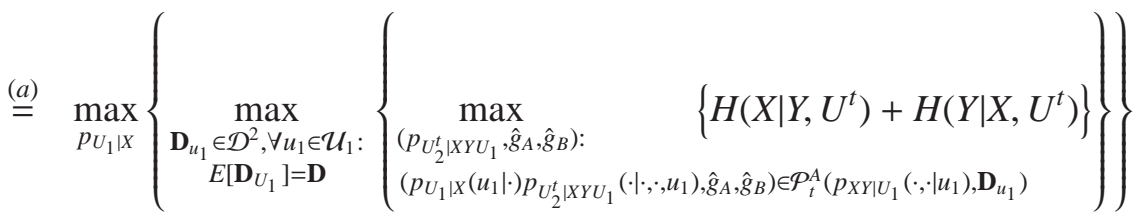

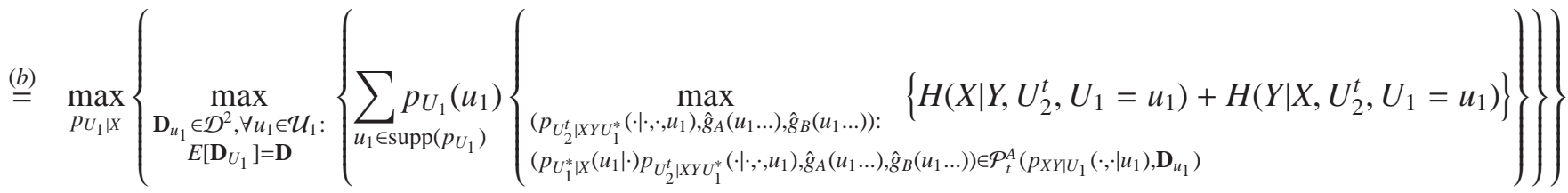

$$
\begin{aligned}
& \stackrel{(c)}{=} \max _{p_{U_{1} \mid X}}\left\{\max _{\substack{\mathbf{D}_{u_{1}} \in \mathcal{D}^{2}, \forall u_{1} \in \mathcal{U}_{1} \\
E\left[\mathbf{D}_{U_{1}}\right]=\mathbf{D}}}\left\{\sum_{u_{1} \in \operatorname{supp}\left(p_{U_{1}}\right)} p_{U_{1}}\left(u_{1}\right) \rho_{t-1}^{B}\left(p_{X Y \mid U_{1}}\left(\cdot, \cdot \mid u_{1}\right), \mathbf{D}_{u_{1}}\right)\right\}\right\} \text {. }
\end{aligned}
$$

In step (a) we replaced the overall distortion constraints $E\left[d_{A}\left(X, Y, \hat{g}_{A}\left(U^{t}, X\right)\right)\right] \leq D_{A}$ and $E\left[d_{B}\left(X, Y, \hat{g}_{B}\left(U^{t}, Y\right)\right)\right] \leq$ $D_{B}$ by the individual distortion constraints $E\left[d_{A}\left(X, Y, \hat{g}_{A}\left(U_{1}, U_{2}^{t}, X\right)\right) \mid U_{1}=u_{1}\right] \leq D_{A, u_{1}}$ and $E\left[d_{B}\left(X, Y, \hat{g}_{B}\left(U_{1}, U_{2}^{t}, Y\right)\right) \mid U_{1}=\right.$ $\left.u_{1}\right] \leq D_{B, u_{1}}$ for all $u_{1} \in \mathcal{U}_{1}$, and maximized the objective function over all the possibilities of the individual distortion levels $\mathbf{D}_{u_{1}}$ satisfying $E\left[\mathbf{D}_{U_{1}}\right]=\sum_{u_{1}} \mathbf{D}_{u_{1}} p_{U_{1}}\left(u_{1}\right)=\mathbf{D}$. Step (b) follows from the "law of total conditional entropy" with the additional observation that conditioned on $U_{1}=u_{1},\left(H\left(X \mid Y, U_{2}^{t}, U_{1}=u_{1}\right)+H\left(Y \mid X, U_{2}^{t}, U_{1}=u_{1}\right)\right)$ only depends on $p_{U_{2}^{t} \mid X Y U_{1}}\left(\cdot \cdot \cdot, \cdot, u_{1}\right), \hat{g}_{A}\left(u_{1}, \ldots\right)$, and $\hat{g}_{B}\left(u_{1}, \ldots\right)$. Step (c) is due to the observation that for a fixed $p_{U_{1} \mid X}$, conditioned on $U_{1}=u_{1},\left(p_{U_{1} \mid X} p_{U_{2}^{t} \mid X Y U_{1}}, \hat{g}_{A}, \hat{g}_{B}\right) \in \mathcal{P}_{t}^{A}\left(p_{X Y, u_{1}}, \mathbf{D}_{u_{1}}\right)$ iff $\left(p_{U_{2}^{t} \mid X Y U_{1}}, \hat{g}_{A}, \hat{g}_{B}\right) \in \mathcal{P}_{t-1}^{B}\left(p_{X Y, u_{1}}, \mathbf{D}_{u_{1}}\right)$, where $p_{X Y, u_{1}}:=p_{X Y \mid U_{1}}\left(\cdot, \cdot \mid u_{1}\right)$. As discussed in Remark 9 in Appendix $\mathrm{A}$ for all $u_{1} \in \operatorname{supp}\left(p_{U_{1}}\right), p_{X Y \mid U_{1}}\left(\cdot, \cdot \mid u_{1}\right) \in \mathcal{P}_{Y \mid X}\left(p_{X Y}\right)$.

(ii) For an arbitrary $q_{X Y} \in \mathcal{P}_{X Y}$, consider two arbitrary joint pmfs $p_{X Y, 1}, p_{X Y, 0} \in \mathcal{P}_{Y \mid X}\left(q_{X Y}\right)$, and two arbitrary distortion vectors $\mathbf{D}_{1}, \mathbf{D}_{0} \in \mathcal{D}^{2}$. For every $\lambda \in(0,1)$, let $\left(p_{X Y, \lambda}, \mathbf{D}_{\lambda}\right):=\lambda\left(p_{X Y, 1}, \mathbf{D}_{1}\right)+\bar{\lambda}\left(p_{X Y, 0}, \mathbf{D}_{0}\right)$. We need to show that $\rho_{t}^{A}\left(p_{X Y, \lambda}, \mathbf{D}_{\lambda}\right) \geq \lambda \rho_{t}^{A}\left(p_{X Y, 1}, \mathbf{D}_{1}\right)+\bar{\lambda} \rho_{t}^{A}\left(p_{X Y, 0}, \mathbf{D}_{0}\right)$. For $i=0,1$, let $p_{U_{1} \mid X, i}$ and $\left\{\mathbf{D}_{u_{1}, i}\right\}_{u_{1} \in \mathcal{U}_{1}}$ be the conditional pmf and individual distortion vectors that maximize the objective function in (12) for source pmf $p_{X Y, i}$ and distortion level $\mathbf{D}_{i}$. Let $p_{U_{1}, i}$ and $p_{X Y \mid U_{1}, i}$ denote, respectively, the $U_{1}$-marginal and conditional pmfs of $p_{X Y, i} p_{U_{1} \mid X, i}$. Therefore we have $\rho_{t}^{A}\left(p_{X Y, i}, \mathbf{D}_{i}\right)=\sum_{u_{1}} p_{U_{1}, i}\left(u_{1}\right) \rho_{t-1}^{B}\left(p_{X Y \mid U_{1}, i}\left(\cdot, \cdot \mid u_{1}\right), \mathbf{D}_{u_{1}, i}\right)$. Define a new auxiliary variable $V \in \mathcal{U}_{1} \times\{0,1\}$ such that $p_{V}\left(u_{1}, 1\right):=\lambda p_{U_{1}, 1}\left(u_{1}\right)$ and $p_{V}\left(u_{1}, 0\right):=\bar{\lambda} p_{U_{1}, 0}\left(u_{1}\right)$. Let $p_{X Y \mid V}\left(\cdot, \cdot \mid u_{1}, i\right):=p_{X Y \mid U_{1}, i}\left(\cdot, \cdot \mid u_{1}\right)$. Then the $X Y$-marginal pmf of $p_{V} p_{X Y \mid V}$ is $p_{X Y, \lambda}$. Let the individual distortion vectors $\mathbf{D}_{v}$ for $v=\left(u_{1}, i\right)$ be $\mathbf{D}_{u_{1}, i}$. The overall distortion vector 
is $E\left[\mathbf{D}_{V}\right]=\mathbf{D}_{\lambda}$. We have,

$$
\begin{aligned}
\lambda & \rho_{t}^{A}\left(p_{X Y, 1}, \mathbf{D}_{1}\right)+\bar{\lambda} \rho_{t}^{A}\left(p_{X Y, 0}, \mathbf{D}_{0}\right) \\
& =\lambda \sum_{u_{1}} p_{U_{1}, 1}\left(u_{1}\right) \rho_{t-1}^{B}\left(p_{X Y \mid U_{1}, 1}\left(\cdot, \cdot \mid u_{1}\right), \mathbf{D}_{u_{1}, 1}\right)+\bar{\lambda} \sum_{u_{1}} p_{U_{1}, 0}\left(u_{1}\right) \rho_{t-1}^{B}\left(p_{X Y \mid U_{1}, 0}\left(\cdot, \cdot \mid u_{1}\right), \mathbf{D}_{u_{1}, 0}\right) \\
& =\sum_{u_{1}, i} p_{V}\left(u_{1}, i\right) \rho_{t-1}^{B}\left(p_{X Y \mid V}\left(\cdot, \cdot \mid u_{1}, i\right), \mathbf{D}_{u_{1}, i}\right) \\
& \leq \rho_{t}^{A}\left(p_{X Y, \lambda}, \mathbf{D}_{\lambda}\right),
\end{aligned}
$$

where the last step is because (12) holds for source pmf $p_{X Y, \lambda}$ and distortion level $\mathbf{D}_{\lambda}$.

(iii) For all $p_{X Y} \in \mathcal{P}_{Y \mid X}\left(q_{X Y}\right)$, we have

$$
\begin{aligned}
\rho_{t}^{A}\left(p_{X Y}, \mathbf{D}\right) & \stackrel{(d)}{=} \max _{p_{U_{1} \mid X}}\left\{\max _{\substack{\forall u_{1} \in \mathcal{U}_{1}, \mathbf{D}_{u_{1}} \in \mathcal{D}^{2}: \\
E\left[\mathbf{D}_{U_{1}}\right]=\mathbf{D}}}\left\{\sum_{u_{1}} p_{U_{1}}\left(u_{1}\right) \rho_{t-1}^{B}\left(p_{X Y \mid U_{1}}\left(\cdot, \cdot \mid u_{1}\right), \mathbf{D}_{u_{1}}\right)\right\}\right\} \\
& \stackrel{(e)}{\leq} \max _{p_{U_{1} \mid X}}\left\{\max _{\substack{\forall u_{1} \in \mathcal{U}_{1}, \mathbf{D}_{u_{1}} \in \mathcal{D}^{2}: \\
E\left[\mathbf{D}_{U_{1}}\right]=\mathbf{D}}}\left\{\sum_{u_{1}} p_{U_{1}}\left(u_{1}\right) \rho\left(p_{X Y \mid U_{1}}\left(\cdot, \cdot \mid u_{1}\right), \mathbf{D}_{u_{1}}\right)\right\}\right\} \\
& \stackrel{(f)}{\leq} \rho\left(p_{X Y}\right),
\end{aligned}
$$

where the equality (d) follows from (12), the inequality (e) is true because $\rho_{t-1}^{B} \leq \rho$, and the final inequality (f) is true due to the following reasons: (i) $p_{X Y \mid U_{1}}\left(\cdot, \cdot \mid u_{1}\right) \in \mathcal{P}_{Y \mid X}\left(p_{X Y}\right) \subseteq \mathcal{P}_{Y \mid X}\left(q_{X Y}\right)$ due to Remarks 2 and 9 , (ii) $\rho$ is concave on $\mathcal{P}_{Y \mid X}\left(q_{X Y}\right) \times \mathcal{D}^{2}$, and (iii) Jensen's inequality.

(iv) This part is proved by reversing the roles of terminal $A$ and $B$ in parts (i) - (iii).

\section{APPENDIX E}

Proofs of Propositions in SectionVIII

Proof of Proposition 4. Given a general $p_{U \mid X}$ and $\hat{g}_{B}$ satisfying the original constraint in (14), we will construct $U^{*}$ satisfying the stronger constraints in Proposition 4 with an objective function that is not less than the original one as follows.

Without loss of generality, we assume $\operatorname{supp}\left(p_{U}\right)=\mathcal{U}$. For $i=0,1$, let $\mathcal{U}_{i}:=\left\{u \in \mathcal{U}: p_{X \mid U}(i \mid u)=1\right\}$. Let $\mathcal{U}_{e}:=$ $\left\{u \in \mathcal{U}: p_{X \mid U}(1 \mid u) \in(0,1)\right\}$. Then $\left\{\mathcal{U}_{1}, \mathcal{U}_{0}, \mathcal{U}_{e}\right\}$ forms a partition of $\mathcal{U}$. For each $u \in \mathcal{U}_{e}$, since $p_{X Y \mid U}(x, y \mid u)>0$ for all $(x, y) \in\{0,1\}^{2}$, it follows that $\hat{g}_{B}(u, y=0)=\hat{g}_{B}(u, y=1)=e$ must hold, because otherwise $E\left(d\left(X, \hat{g}_{B}(U, Y)\right)\right)=\infty$. But for every $u \in \mathcal{U}_{i}, i=0,1, \hat{g}_{B}(u, y)$ may equal $i$ or $e$ but not $(1-i)$ to get a finite distortion. When we replace $\hat{g}_{B}$ by

$$
\hat{g}_{B}^{*}(u, y)= \begin{cases}i, & \text { if } u \in \mathcal{U}_{i}, i=0,1, \\ e, & \text { if } u \in \mathcal{U}_{e},\end{cases}
$$

the distortion for $u \in \mathcal{U}_{i}, i=0,1$, is reduced to zero, and the distortion for $u \in \mathcal{U}_{e}$ remains unchanged. Therefore we have $E\left(d\left(X, \hat{g}_{B}^{*}(U, Y)\right)\right) \leq E\left(d\left(X, \hat{g}_{B}(U, Y)\right)\right) \leq D$. We note that $\hat{g}_{B}^{*}(U, Y)$ is completely determined by $U$. Let $U^{*}:=\hat{g}_{B}^{*}(U, Y)$. Then $U^{*}=i$ iff $U \in \mathcal{U}_{i}, i=\{0,1, e\}$. The objective function $H(X \mid Y, U)+H(Y \mid X)=$ $H\left(X \mid Y, U, U^{*}\right)+H(Y \mid X) \leq H\left(X \mid Y, U^{*}\right)+H(Y \mid X)$, which completes the proof. 


\section{Proof of Proposition 6 .}

For a fixed $p_{X Y}, H(X \mid Y, U)+H(Y \mid X)$ is concave with respect to $p_{X Y U}$ and therefore also $p_{U \mid X}$. Since $p_{U \mid X}$ is linear with respect to $\left(\alpha_{0 e}, \alpha_{1 e}\right), \psi\left(p_{X Y}, \alpha_{0 e}, \alpha_{1 e}\right)=H(X \mid Y, U)+H(Y \mid X)$ is concave with respect to $\left(\alpha_{0 e}, \alpha_{1 e}\right)$.

The maximum in (16) can be achieved along the axis of symmetry given by $\alpha_{1 e}=\alpha_{0 e}$ because (i) $\psi$ and $\phi$ are both symmetric with respect to $\alpha_{0 e}$ and $\alpha_{1 e}$, i.e., $\psi\left(p_{X Y}, \alpha_{0 e}, \alpha_{1 e}\right)=\psi\left(p_{X Y}, \alpha_{1 e}, \alpha_{0 e}\right)$ and $\phi\left(p_{X Y}, \alpha_{0 e}, \alpha_{1 e}\right)=\phi\left(p_{X Y}, \alpha_{1 e}, \alpha_{0 e}\right)$, and (ii) $\psi\left(p_{X Y}, \alpha_{0 e}, \alpha_{1 e}\right)$ is a concave function of $\left(\alpha_{0 e}, \alpha_{1 e}\right)$. When $D \in[0,1], \rho_{1}^{A}$ can be further simplified as follows.

$$
\rho_{1}^{A}\left(p_{X Y}, D\right)=\max _{\alpha_{0 e}=\alpha_{1 e} \in[0, D]} \psi\left(p_{X Y}, \alpha_{0 e}, \alpha_{1 e}\right)=(1+D) h_{2}(p),
$$

which completes the proof.

Proof of Proposition 7. For the joint pmf $p_{X \mid Y} p_{Y, 1}$ summarized in Table II functions $\psi$ and $\eta$ simplify even further TABLE II

JOINT DISTRIBUTION $p_{X \mid Y} p_{Y, 1}$

\begin{tabular}{|c|c|c|}
\hline$p_{X \mid Y} p_{Y, 1}$ & $y=0$ & $y=1$ \\
\hline$x=0$ & $\bar{p} \bar{q}$ & $p q$ \\
\hline$x=1$ & $p \bar{q}$ & $\bar{p} q$ \\
\hline
\end{tabular}

to special functions of $\left(p, q, \alpha_{0 e}, \alpha_{1 e}\right)$ as follows:

$$
\begin{aligned}
C\left(p, q, \alpha_{0 e}, \alpha_{1 e}\right)= & \psi\left(p_{X \mid Y} p_{Y, 1}, \alpha_{0 e}, \alpha_{1 e}\right) \\
= & \bar{q}\left(\bar{p} \alpha_{0 e}+p \alpha_{1 e}\right) h_{2}\left(\frac{\bar{p} \alpha_{0 e}}{\bar{p} \alpha_{0 e}+p \alpha_{1 e}}\right) \\
& +q\left(p \alpha_{0 e}+\bar{p} \alpha_{1 e}\right) h_{2}\left(\frac{p \alpha_{0 e}}{p \alpha_{0 e}+\bar{p} \alpha_{1 e}}\right) \\
& +(\bar{p} \bar{q}+p q) h_{2}\left(\frac{\bar{p} \bar{q}}{\bar{p} \bar{q}+p q}\right) \\
& +(\bar{p} q+p \bar{q}) h_{2}\left(\frac{\bar{p} q}{\bar{p} q+p \bar{q}}\right), \\
\eta\left(p, q, \alpha_{0 e}, \alpha_{1 e}\right)= & \phi\left(p_{X \mid Y} p_{Y, 1}, \alpha_{0 e}, \alpha_{1 e}\right) \\
= & (\bar{p} \bar{q}+p q) \alpha_{0 e}+(\bar{p} q+p \bar{q}) \alpha_{1 e} .
\end{aligned}
$$

Observe that $C\left(p, q, \alpha_{0 e}, \alpha_{1 e}\right)=C\left(p, \bar{q}, \alpha_{1 e}, \alpha_{0 e}\right)$, and $\eta\left(p, q, \alpha_{0 e}, \alpha_{1 e}\right)=\eta\left(p, \bar{q}, \alpha_{1 e}, \alpha_{0 e}\right)$ hold. Therefore we have

$$
\begin{aligned}
\rho_{1}^{A}\left(p_{X \mid Y} p_{Y, 2}, D\right) & =\max _{\substack{\alpha_{e}, \alpha_{1 e} \in[0,1]: \\
\eta\left(p, \bar{q}, \alpha_{0 e}, \alpha_{1 e}\right) \leq D}} C\left(p, \bar{q}, \alpha_{0 e}, \alpha_{1 e}\right) \\
& =\max _{\substack{\alpha_{0 e}, \alpha_{1 e} \in[0,1]: \\
\eta\left(p e q, \alpha_{1 e}, \alpha_{0 e}\right) \leq D}} C\left(p, q, \alpha_{1 e}, \alpha_{0 e}\right) \\
& =\rho_{1}^{A}\left(p_{X \mid Y} p_{Y, 1}, D\right) .
\end{aligned}
$$

It follows that

$$
\begin{aligned}
\frac{\rho_{1}^{A}\left(p_{X \mid Y} p_{Y, 1}, D\right)+\rho_{1}^{A}\left(p_{X \mid Y} p_{Y, 2}, D\right)}{2} & =\rho_{1}^{A}\left(p_{X \mid Y} p_{Y, 1}, D\right) \\
& \geq C\left(p, q, \alpha_{0 e}, 1\right)
\end{aligned}
$$

holds for $D=\eta\left(p, q, \alpha_{0 e}, 1\right)$.

Proof of Proposition 8 . 
Since $D=\eta\left(p, q, \alpha_{0 e}, 1\right) \in[0,1]$ always holds, we have $\rho_{1}^{A}\left(p_{X Y}, D\right)=(1+D) h_{2}(p)$ due to (17). We will show that for any fixed $q \in(0,1 / 2)$ and $\alpha_{0 e} \in(0,1), \lim _{p \rightarrow 0} C\left(p, q, \alpha_{0 e}, 1\right) / h_{2}(p)>\lim _{p \rightarrow 0}(1+D)$ holds, which implies that $\exists p \in(0,1)$ such that $C\left(p, q, \alpha_{0 e}, 1\right) / h_{2}(p)>(1+D)$, which, in turn, implies Proposition 8 . It is convenient to use the following lemma to analyze the limits.

Lemma 3: Let $f(p)$ be a function differentiable around $p=0$ such that $f(0)=0$ and $f^{\prime}(0)>0$. Then

$$
\lim _{p \rightarrow 0} \frac{h_{2}(f(p))}{h_{2}(p)}=f^{\prime}(0)
$$

Proof: Applying the l'Hôpital rule several times, we have

$$
\begin{aligned}
\lim _{p \rightarrow 0} \frac{h_{2}(f(p))}{h_{2}(p)} & =\lim _{p \rightarrow 0} \frac{\ln (1-f(p))-\ln f(p)}{\ln (1-p)-\ln p} f^{\prime}(0) \\
& =\lim _{p \rightarrow 0} \frac{\ln f(p)}{\ln p} f^{\prime}(0) \\
& =\lim _{p \rightarrow 0} \frac{p}{f(p)}\left(f^{\prime}(0)\right)^{2} \\
& =f^{\prime}(0),
\end{aligned}
$$

which completes the proof of Lemma 3

Applying Lemma 3, we have

$$
\begin{aligned}
& \lim _{p \rightarrow 0} \frac{C\left(p, q, \alpha_{0 e}, 1\right)}{h_{2}(p)}=2-q\left(1-\alpha_{0 e}\right) \\
& \lim _{p \rightarrow 0}(1+D)=2-\bar{q}\left(1-\alpha_{0 e}\right), \\
& \lim _{p \rightarrow 0}\left(\frac{C\left(p, q, \alpha_{0 e}, 1\right)}{h_{2}(p)}-(1+D)\right)=(1-2 q)\left(1-\alpha_{0 e}\right) .
\end{aligned}
$$

Therefore for any $\alpha_{0 e} \in(0,1)$ and $q \in(0,1 / 2)$, there exists a small enough $p$ such that $C\left(p, q, \alpha_{0 e}, 1\right)>(1+D)$ holds, which completes the proof.

\section{Proof of Proposition 9 .}

For the rate-distortion tuple $\left(R_{1}, R_{2}, D\right)$ corresponding to the choice of $p_{V_{1} \mid Y}, p_{V_{2} \mid X V_{1}}$ and $\hat{g}_{B}$ described in the proof of Theorem 7 we have (i) $R_{1}=I\left(Y ; V_{1} \mid X\right)=H(Y \mid X)-C_{2}(p, q)$, where $C_{2}(p, q)$ is the sum of the last two terms in (E.2); (ii) $R_{2}=I\left(X ; V_{2} \mid Y, V_{1}\right)=2 h_{2}(p)-C(p, q, \alpha, 1)-R_{1}$; and (iii) $D=\eta(p, q, \alpha, 1)$. It follows that

$$
\begin{aligned}
& \lim _{p \rightarrow 0} \frac{R_{1}}{h_{2}(p)}=0, \\
& \lim _{p \rightarrow 0} \frac{R_{2}}{h_{2}(p)}=2-\lim _{p \rightarrow 0} \frac{C(p, q, \alpha, 1)}{h_{2}(p)}-\lim _{p \rightarrow 0} \frac{R_{1}}{h_{2}(p)}=q(1-\alpha) .
\end{aligned}
$$

Therefore for all $q>0$ and $\alpha \in(0,1)$, we have

$$
\lim _{p \rightarrow 0} \frac{R_{1}}{R_{2}}=0
$$

For the one-message rate-distortion function, we have $R_{\text {sum }, 1}\left(p_{X Y}, D\right)=2 h_{2}(p)-\rho_{1}^{A}\left(p_{X Y}, D\right)$, where $\rho_{1}^{A}\left(p_{X Y}, D\right)$ is given by 177. Therefore we have

$$
\lim _{p \rightarrow 0} \frac{R_{\text {sum }, 1}\left(p_{X Y}, D\right)}{h_{2}(p)}=2-\lim _{p \rightarrow 0} \frac{\rho_{1}^{A}\left(p_{X Y}, D\right)}{h_{2}(p)}=\bar{q}(1-\alpha),
$$

which implies that

$$
\lim _{p \rightarrow 0} \frac{R_{\text {sum }, 1}\left(p_{X Y}, D\right)}{R_{1}+R_{2}}=\frac{\bar{q}}{q}
$$


For any $L>0$, we can always find a small enough $q>0$ such that $\bar{q} / q>L+1$. Due to (E.5) and (E.6), there exists $p>0$ such that $R_{1} / R_{2}<1 / L$ and $R_{\text {sum }, 1} /\left(R_{1}+R_{2}\right)>L$.

Remark 11: The convergence of the limit analyzed in Lemma 3 is actually slow, because the logarithm function increases to infinity slowly. The consequence is that if one chooses a small $q$ to get $R_{\text {sum }, 1} /\left(R_{1}+R_{2}\right)$ close to the limit $\bar{q} / q$, then $p$ needs to be very small. For example, when $q=1 / 10, \alpha_{0 e}=1 / 2, \bar{q} / q=9$, with $p=10^{-200}$, we get $R_{\text {sum }, 1} / R_{\text {sum }, 2}^{*} \approx 8.16$. This, however, does not mean that the benefit of multiple messages only occurs in extreme cases. In computer simulations we have observed that for the erasure distortion, the gain for certain asymmetric sources can be much more than that for the DSBS example analyzed in this paper. The DSBS example was chosen in this paper only because it is easy to analyze.

\section{REFERENCES}

[1] N. Ma and P. Ishwar, "Two-terminal distributed source coding with alternating messages for function computation," in Proc. IEEE Int. Symp. Information Theory, Toronto, Canada, Jul. 6-11, 2008, pp. 51-55, arXiv:0801.0756v4.

[2] N. Ma and P. Ishwar, "Some results on distributed source coding for interactive function computation," IEEE Trans. Inf. Theory, vol. 57, no. 9, pp. 6180 - 6195, Sep. 2011.

[3] A. H. Kaspi, "Two-way source coding with a fidelity criterion," IEEE Trans. Inf. Theory, vol. 31, no. 6, pp. 735-740, Nov. 1985.

[4] A. C. Yao, "Some complexity questions related to distributed computing," in Proc. 11th Annu. ACM Symp. Theory of Computing, Atlanta, GA, Apr. 30-May 2, 1979, pp. 209-213.

[5] E. Kushilevitz and N. Nisan, Communication Complexity. Cambridge: Cambridge University Press, 1997.

[6] A. Orlitsky, "Worst-case interactive communication i: two messages are almost optimal," IEEE Trans. Inf. Theory, vol. 36, no. 5, pp. 1111-1126, Sep. 1990.

[7] — - "Worst-case interactive communication ii: two messages are not optimal," IEEE Trans. Inf. Theory, vol. 37, no. 4, pp. 995-1005, Jul. 1991 .

[8] A. Orlitsky and J. R. Roche, "Coding for computing," IEEE Trans. Inf. Theory, vol. 47, no. 3, pp. 903-917, Mar. 2001.

[9] N. Ma, P. Ishwar, and P. Gupta, "Interactive source coding for function computation in collocated networks," accepted for publication by IEEE Trans. Inf. Theory.

[10] X. Yan, R. W. Yeung, and Z. Zhang, “The capacity region for multi-source multi-sink network coding,” in Proc. IEEE Int. Symp. Information Theory, Nice, France, Jun. 24-29, 2007, pp. 116-120.

[11] R. W. Yeung, Information theory and network coding. New York, New York: Springer Verlag, 2008.

[12] R. T. Rockafellar, Convex Analysis. Princeton: Princeton University Press, 1970.

[13] F. P. Preparata and M. I. Shamos, Computational Geometry: An Introduction. New York, New York: Springer Verlag, 1985.

[14] D. P. Bertsekas, Nonlinear Programming. Belmont, Massachusetts: Athena Scientific, 1995.

[15] A. Cohen and R. Zamir, "Entropy amplification property and the loss for writing on dirty paper," IEEE Tran. Info. Theory, vol. 54, no. 4, pp. 1477-1487, Apr. 2008.

[16] I. Csiszár and J. Körner, Information Theory: Coding Theorems for Discrete Memoryless Systems. Hungary: Akadémiai Kiadó, 1986.

[17] T. M. Cover and J. A. Thomas, Elements of Information Theory. New York: Wiley, 1991. 